\title{
AN INTELLIGENT AIRCRAFT WINDOW SYSTEM FOR VISUAL COMFORT CONTROL
}

by

Jasper Liu

Bachelor of Aerospace Engineering, Ryerson University (2015)

\author{
A thesis \\ presented to Ryerson University \\ in partial fulfillment of the \\ requirements for the degree of \\ Master of Applied Science \\ in the Program of \\ Aerospace Engineering
}

Toronto, Ontario, Canada, 2018

C) Jasper Liu 2018 


\section{AUTHOR'S DECLARATION FOR ELECTRONIC SUBMISSION OF A THESIS}

I hereby declare that I am the sole author of this thesis. This is a true copy of the thesis, including any required final revisions, as accepted by my examiners.

I authorize Ryerson University to lend this thesis to other institutions or individuals for the purpose of scholarly research.

I further authorize Ryerson University to reproduce this thesis by photocopying or by other means, in total or in part, at the request of other institutions or individuals for the purpose of scholarly research.

I understand that my thesis may be made electronically available to the public. 


\title{
AN INTELLIGENT AIRCRAFT WINDOW SYSTEM FOR VISUAL COMFORT CONTROL
}

\author{
Jasper Liu \\ Master of Applied Science, Aerospace Engineering, Ryerson University, Toronto (2018)
}

\begin{abstract}
The purpose of this thesis is to develop an intelligent aircraft window system for visual comfort control based on electrochromic windows that can change the transparency depending on passenger's needs and visual comfort. In the current window system, window transparency is controlled manually. The system developed in this thesis is an automatic system. Under this development, a mock-up is set up to mimic a section of cabin with an electrochromic window along with a simulated sunlight source and several light sensors. To measure visual comfort, the daylight glare index (DGI) is adopted. Based on DGI, a fuzzy logic classifier is developed to evaluate visual comfort/discomfort. This is followed by developing a fuzzy logic controller that can automatically adjust the window transparency based on the measured DGI level and the rate of change of DGI. DGI set point for different individuals, the effect of illuminance and color temperature are also discussed.
\end{abstract}




\section{ACKNOWLEDGEMENTS}

I would like to express my sincere gratitude to Dr. Jeff Xi, my supervisor, for offering an opportunity to take part in his research on aircraft cabin comfort. His guidance, all-around support and his immense knowledge on multiple engineering topics have all been important factors to the success of the completion of my thesis.

I would also like to thank my colleagues, Mr. Gabriel Campos, Dr. Wenbin Wang, and Ms. Upasana Chouduri for providing numerous supports and advices on various problems. Their engineering skills and insightful comments have helped me tackle the most difficult problems that I encountered during the two years of research.

Last but not least, I would like to thank my parents, Mr. Derek Liu and Mrs. Salina Liu, for all-round and unconditional supports during the entire duration of my graduate studies.

Jasper Liu

November, 2017 


\section{TABLE OF CONTENTS}

AUTHOR'S DECLARATION FOR ELECTRONIC SUBMISSION OF A THESIS ................. ii

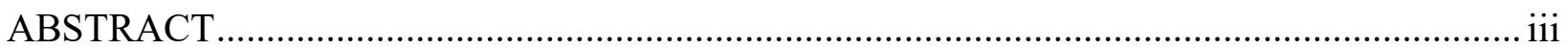

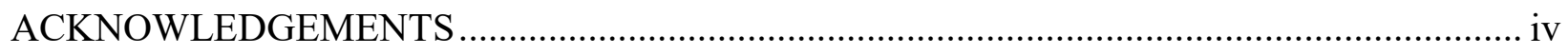

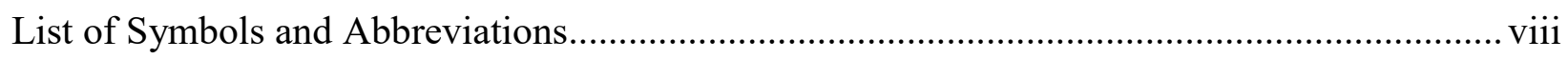

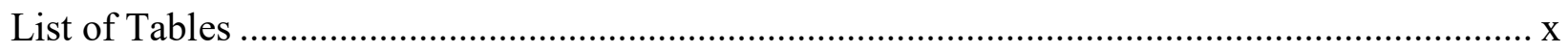

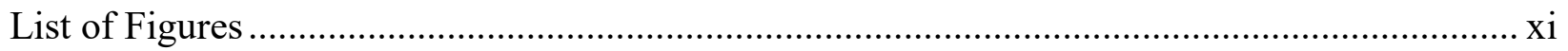

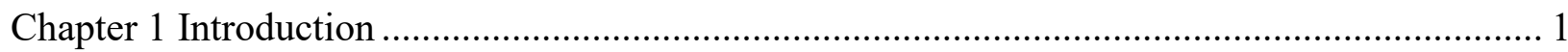

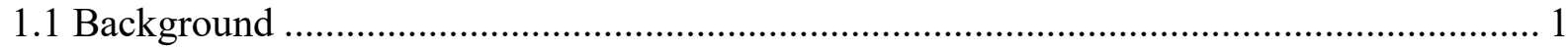

1.2 Problem Statement and Objectives.................................................................... 3

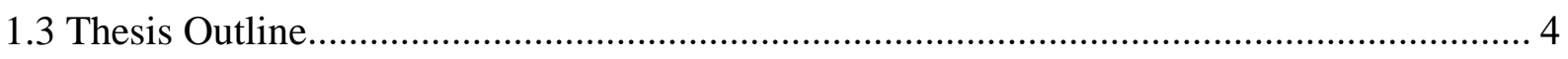

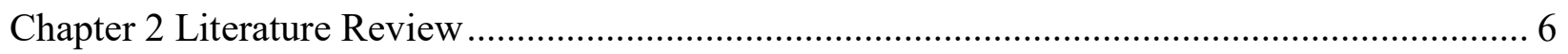

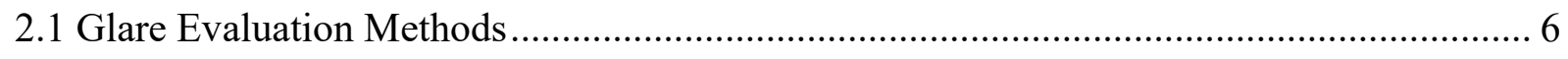

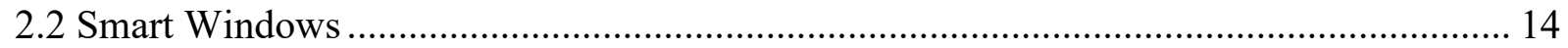

2.3 Effect of Color and Color Temperature on Visual Comfort ....................................... 18

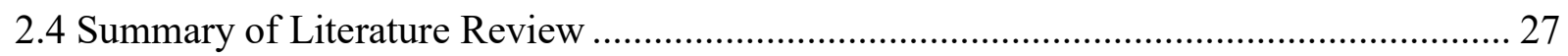

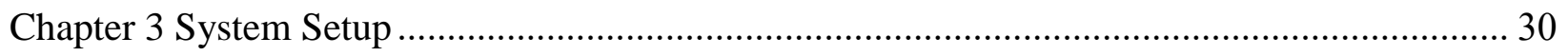

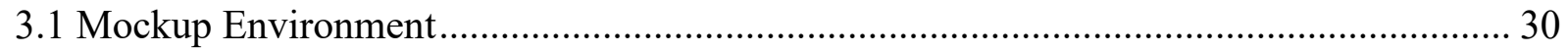

3.2 Testing the Properties of Electrochromic Window ................................................. 35

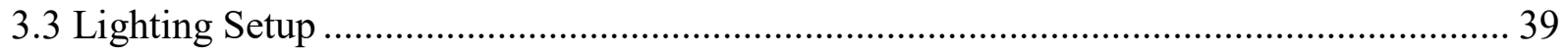

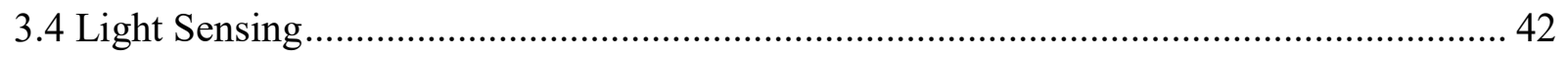

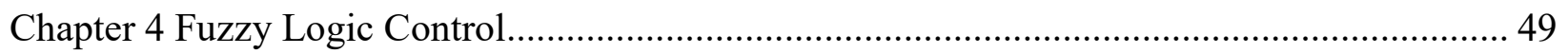




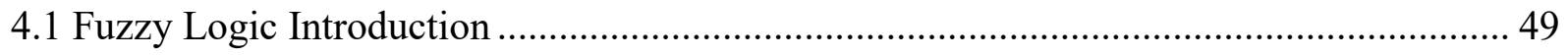

4.2 Fuzzy Logic Classification ........................................................................................... 50

4.3 Initial Fuzzy Logic Controller Design.......................................................................... 52

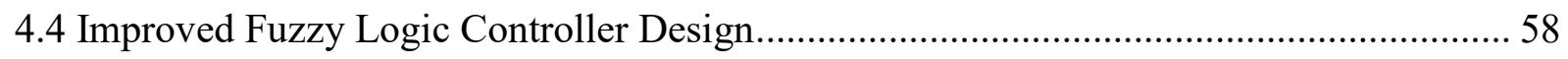

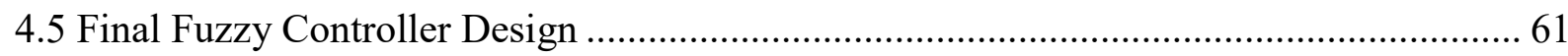

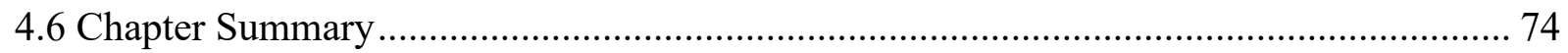

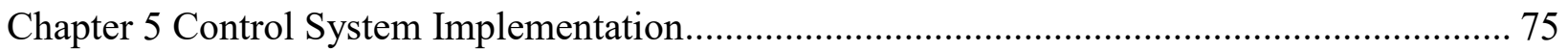

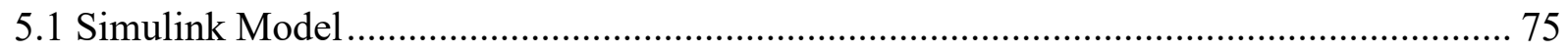

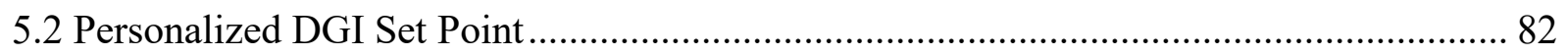

5.3 Investigation of Horizontal Illuminance and Color Temperature ………………….......... 95

5.3.1 Horizontal Illuminance Versus Color Temperature Data Collection Method ............. 95

5.3.2 Results of Participants' Selections and Their Verbal Responses ................................... 97

5.3.3 Discussion of Results of Participants' Responses ....................................................... 98

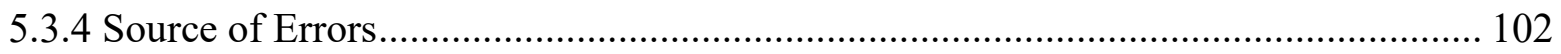

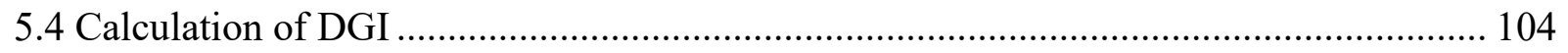

5.5 Re-location of Light Sensors ....................................................................................... 105

5.5.1 Changing Angle of Light Sensor Pair ....................................................................... 107

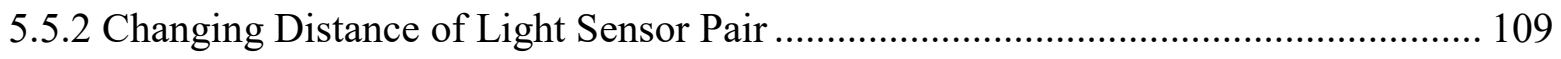

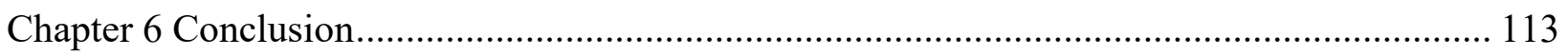

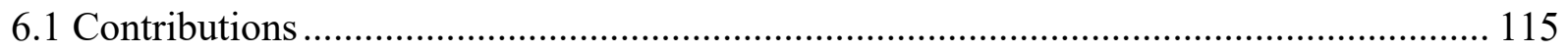




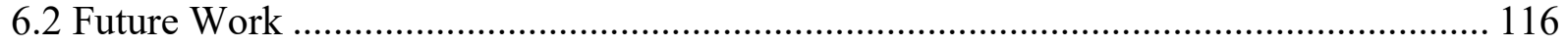

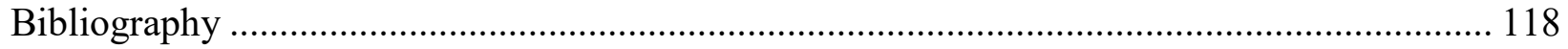




\section{List of Symbols and Abbreviations}

\begin{tabular}{|c|c|}
\hline$a$ & Width of window \\
\hline$a^{\prime}$ & Wider opening of pyramid \\
\hline ANFIS & Adaptive neural-fuzzy inference system \\
\hline$b$ & Height of window \\
\hline$d$ & Perpendicular distance from the window to the sensor shielded by pyramid \\
\hline$d^{\prime}$ & Depth of pyramid \\
\hline DGI & Daylight glare index \\
\hline $\mathrm{DGI}_{\mathrm{m}}$ & Measured/detected daylight glare index value \\
\hline $\mathrm{DGI}_{\mathrm{N}}$ & New daylight glare index \\
\hline $\mathrm{DGI}_{\mathrm{O}}$ & Daylight glare index as set point of fuzzy logic control input \\
\hline DGISP & Intermediate daylight glare index set point \\
\hline $\mathrm{EC}$ & Electrochromic \\
\hline $\mathrm{E}_{\mathrm{v}}$ & Vertical luminance \\
\hline FIS & Fuzzy inference system \\
\hline GR & Glare rating \\
\hline GUI & Graphical user interface \\
\hline IMF & Fuzzy logic input membership function \\
\hline Ladaptation & Adaptation luminance \\
\hline Lexterior & Average vertical unshielded luminance of the outdoor \\
\hline Lwindow & $\begin{array}{l}\text { Average vertical luminance of the window that is calculated from the reading of } \\
\text { the sensor with a shielding pyramid }\end{array}$ \\
\hline M & Mirek, inverse of color temperature multiply by 106 \\
\hline $\mathrm{MF}$ & Fuzzy logic membership function \\
\hline $\mathrm{OMF}$ & Fuzzy logic output membership function \\
\hline PDLC & Polymer dispersed liquid crystal \\
\hline RQ & RoboteQ controller \\
\hline$s^{\prime}$ & Distance between window to the wider opening of pyramid \\
\hline SISO & Single input single output \\
\hline SusP & Suspended particle \\
\hline UGR & Unified glare index \\
\hline
\end{tabular}


$\alpha$
New/next voltage applied to electrochromic window

Current voltage applied to electrochromic window

Required voltage increase/decrease to change tint state of electrochromic window

Photopic vision function

Scotopic vision function

Length of smaller opening of pyramid

Solid angle subtended by the window to the point of observation

Solid angular subtence of the source modified for the effect of the position of its elements in different parts of the field of view

Configuration factor of the window from the observation place

Angle between sensor pair and the horizontal 


\section{List of Tables}

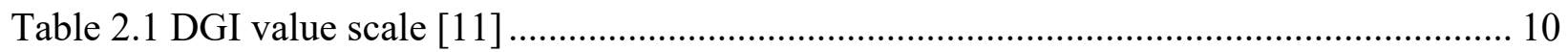

Table 2.2 Illuminance values (lux) at various outdoor scenarios [17] ...................................... 10

Table 2.3 Illuminance values (lux) at various indoor scenarios [18]......................................... 10

Table 2.4 Properties of three different types of smart windows ............................................... 15

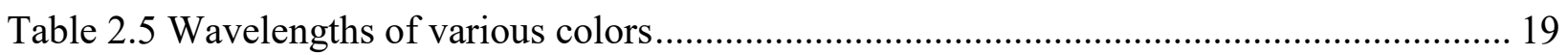

Table 2.6 Color temperatures of various light sources [35]...................................................... 20

Table 2.7 Perception of various color temperatures concluded by Bodman [42] ........................ 22

Table 2.8 Preferred illuminance levels and color temperatures for various activity settings [43] 22

Table 4.1 Fuzzy rules for the improved fuzzy logic controller.................................................. 59

Table 4.2 Transition times for various dimming and clearing sections as shown in Figure 4.17.63

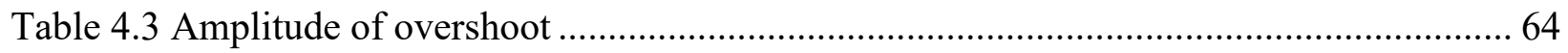

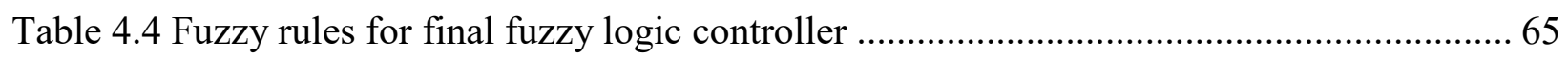

Table 5.1 Suggested DGI bias values at various glare tolerance levels......................................... 79

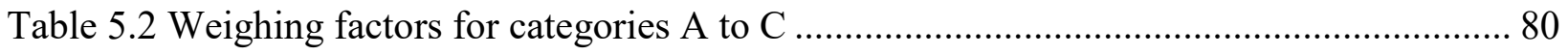

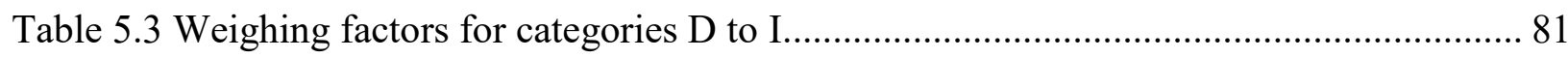

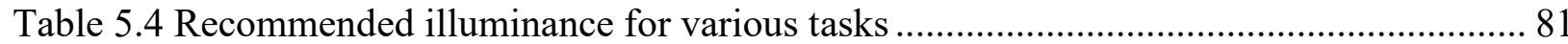

Table 5.5 DGISP for various activities performed by different age groups.................................. 84

Table 5.6 Output membership functions for the fuzzy inference system computed by ANFIS ... 91

Table 5.7 Illuminance values (lux) at various indoor scenarios .................................................... 98

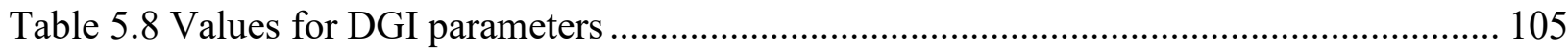

Table 5.9 Maximum DGI difference for angle adjustment at various distances ......................... 108 


\section{List of Figures}

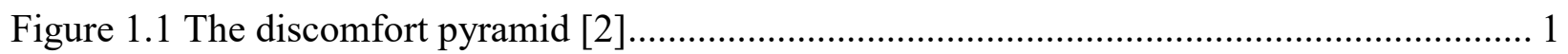

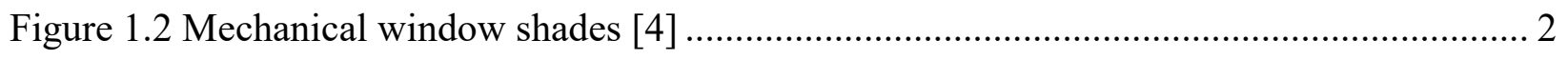

Figure 1.3 Electrochromic windows of various dimming levels [5] .......................................... 2

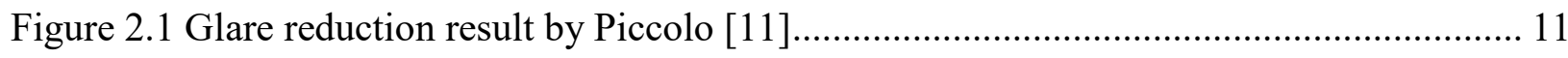

Figure 2.2 Original picture (left) and pixelated picture with luminance values (right) [19] ….... 12

Figure 2.3 Processed image for finding high contrast zone (left), fuzzy logic for evaluating

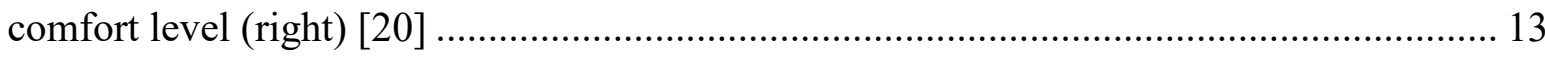

Figure 2.4 Illustration of the operation of a suspended particle device [25] ............................. 15

Figure 2.5 Illustration of the operation of a polymer dispersed liquid crystal device [26].......... 16

Figure 2.6 Illustration of the operation of an electrochromic window (provided by Gentex) ...... 17

Figure 2.7 Photopic and scoptopic vision response curves [30] ................................................ 19

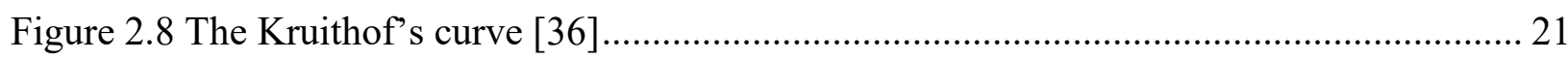

Figure 2.9 DISO fuzzy logic system proposed by Saravanan [44] ........................................... 24

Figure 2.10 Response of light bulb under bright (left), moderate (center) and dark (right) environment [44]. 24

Figure 2.11 Comparison of thermal control results using adaptive (top) and non-adaptive (bottom)

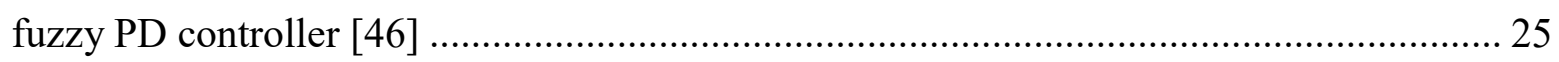

Figure 2.12 Graphical representation of utility function [49] ............................................... 26

Figure 2.13 User preference consideration using fuzzy logic and utility function for lighting control [49]

Figure 2.14 Combination of fuzzy control and PID control for vertical illumination adjustment [50]

Figure 3.1 Bombardier Aerospace Global 7000 cabin environment (provided by Bombardier

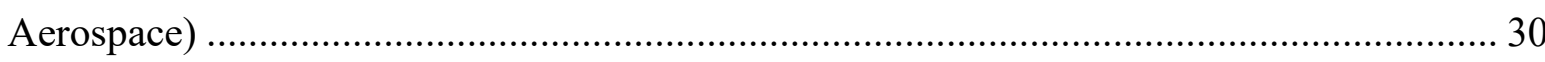

Figure 3.2 Bombardier Aerospace Global 7000 seating arrangement ......................................... 31

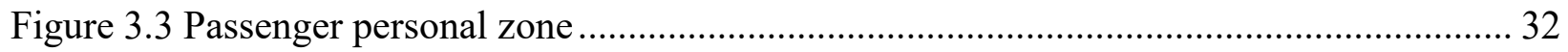

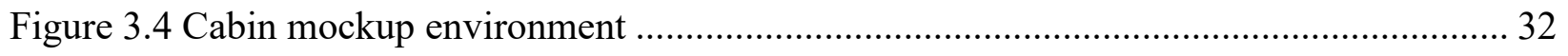

Figure 3.5 View from inside the mock up ............................................................................ 33

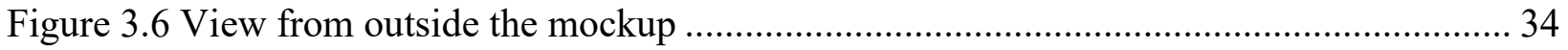

Figure 3.7 Placement of electrochromic window in aircraft cabin (picture provided by Gentex) 35 


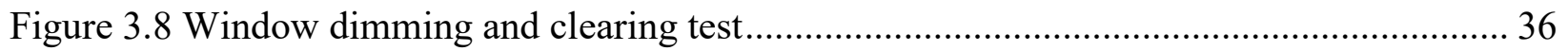

Figure 3.9 Electrochromic window from fully dimmed to clear .......................................... 37

Figure 3.10 Electrochromic window from clear to fully dimmed ......................................... 37

Figure 3.11 Electrochromic window manual controller provided by Gentex .......................... 38

Figure 3.12 Picture of RoboteQ motor controller connecting to the electrochromic window ..... 38

Figure 3.13 RoboteQ motor controller output voltage vs percentage of controller input voltage 39

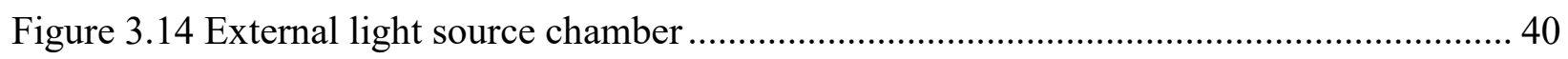

Figure 3.15 Inside the external light source chamber ..................................................... 41

Figure 3.16 Illustration of optics enhancement for the light chamber ................................... 41

Figure 3.17 View from inside the mock up without optics enhancement (left) vs with optics

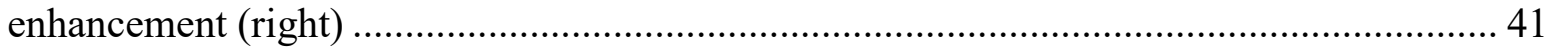

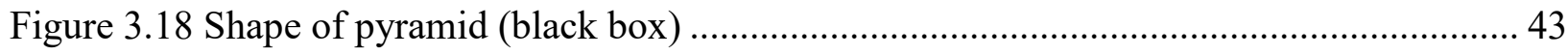

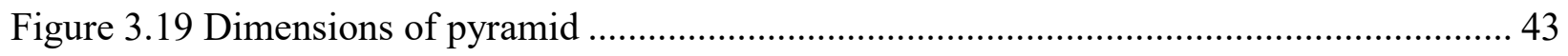

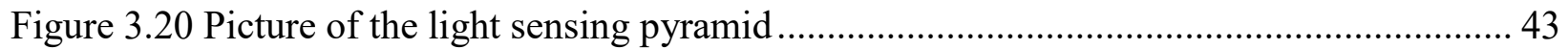

Figure 3.21 Placement of the three sensors and the pyramid [7] ......................................... 44

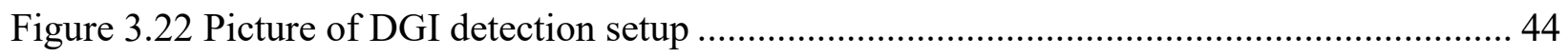

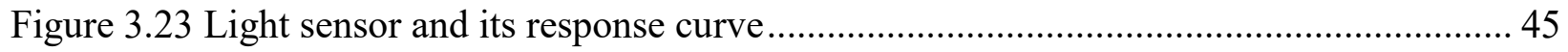

Figure 3.24 An 8 channel 4-20mA data acquisition board .................................................. 45

Figure 3.25 AMS302 photo diode response sensitivity vs wavelength ................................. 46

Figure 3.26 Circuit diagram for Panasonic AMS302 photodiode .......................................... 46

Figure 3.27 Resistance too low (left) vs resistance too high (right) for photo diode circuit ....... 47

Figure 3.28 A proper choice of resistor for photo diode circuit $-520 \Omega$................................. 48

Figure 3.29 Photocurrent versus horizontal illuminance ................................................... 48

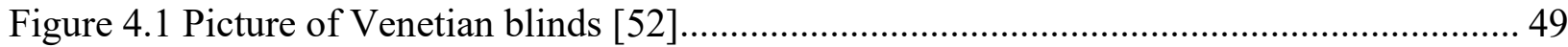

Figure 4.2 Fuzzy logic window tint classification result ................................................ 50

Figure 4.3 Fuzzy logic membership functions with DGI classification .................................. 50

Figure 4.4 Control diagram with version 1 fuzzy logic controller - pure linguistic approach ..... 53

Figure 4.5 Input membership functions of version 1 fuzzy logic controller - Test 1................ 54

Figure 4.6 Output membership functions of version 1 fuzzy logic controller - Test 1 ............. 54

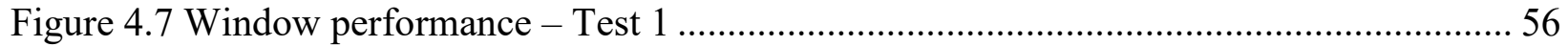

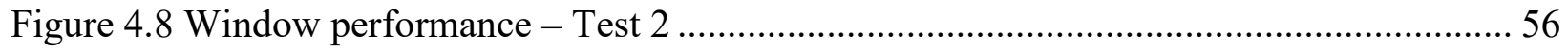


Figure 4.9 $\Delta \mathrm{V}$ output versus DGI 57

Figure 4.10 Input membership functions of version 2 fuzzy logic controller - Test 2............. 57

Figure 4.11 Output membership functions of version 2 fuzzy logic controller - Test 2 ........... 58

Figure 4.12 Control diagram with version 2 controller - linguistic approach with practical control

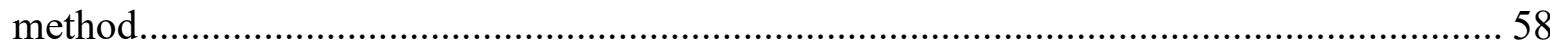

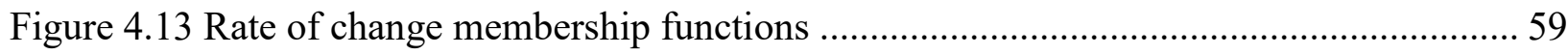

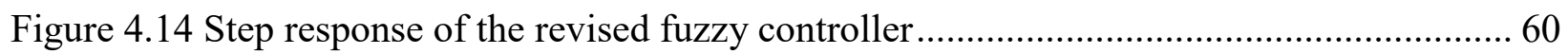

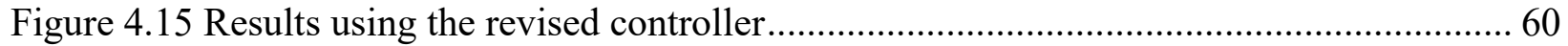

Figure 4.16 Control diagram for version 3 controller - most practical approach (PD controller) 62

Figure 4.17 Results using final fuzzy logic controller version ........................................... 62

Figure 4.18 Input membership function “error” for final fuzzy logic controller ........................ 64

Figure 4.19 Input membership function "rate of change" for final fuzzy logic controller .......... 64

Figure 4.20 Output membership function "window operation" for final fuzzy logic controller.. 64

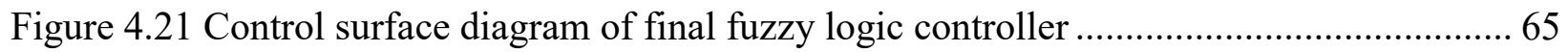

Figure 4.22 Control surface diagram categorized into five sections .....................................6 66

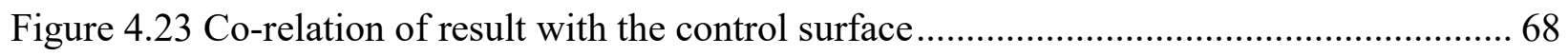

Figure 4.24 Results of using fuzzy logic controller with 25 rules and triangular shaped membership functions.

Figure 4.25 Results of using fuzzy logic controller with 25 rules and Gaussian shaped membership functions 69

Figure 4.26 Demonstration - triangular input membership functions.................................... 70

Figure 4.27 Demonstration - triangular output membership functions................................. 70

Figure 4.28 Demonstration - Gaussian input membership functions .................................... 71

Figure 4.29 Demonstration - Gaussian output membership functions ................................. 71

Figure 4.30 Demonstration - output results using triangular input and output membership

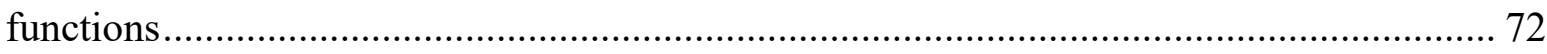

Figure 4.31 Demonstration - output results using Gaussian input and output membership functions. 72

Figure 4.32 Designed fuzzy PD controller output curve - output vs error ............................. 73

Figure 4.33 Designed fuzzy PD controller output curve - output vs rate of change................... 73

Figure 5.1 Hardware connection wiring diagram ........................................................... 75 


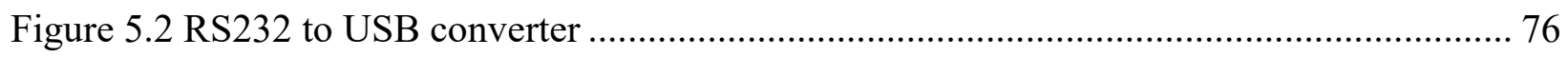

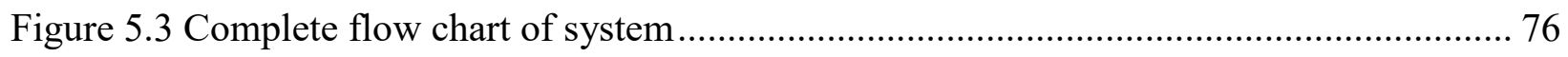

Figure 5.4 Software implementation - Simulink model.............................................................. 77

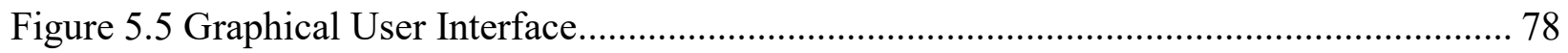

Figure 5.6 Electrochromic window control integrated with user tolerance level ........................ 79

Figure 5.7 Full electrochromic window control integrated with influence of human perception 83

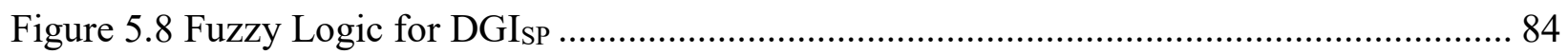

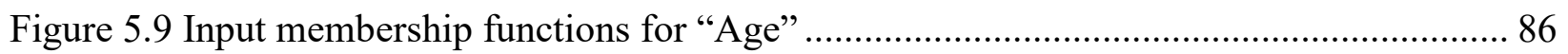

Figure 5.10 Input membership functions for "Activity" ........................................................... 86

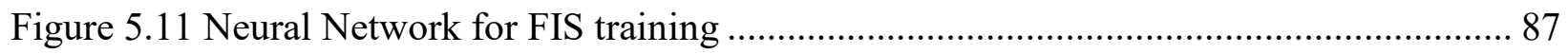

Figure 5.12 Moving average of various DGISP level selected by users ..................................... 88

Figure 5.13 Improper control surface (left) vs proper control surface (right) ………………....... 88

Figure 5.14 Input membership functions for "Activity" generated by ANFIS.............................. 89

Figure 5.15 Input membership functions for "Age" generated by ANFIS …………………….... 89

Figure 5.16 Result from fuzzy logic DGI output for various ages after ANFIS training ............. 90

Figure 5.17 Input membership functions for "Activity" after adjustment ................................... 91

Figure 5.18 Input membership functions for "Age" after adjustment ......................................... 91

Figure 5.19 Control surface diagram of fuzzy inference system after adjustment ...................... 92

Figure 5.20 Result from fuzzy logic DGI output for various ages after adjustment ................... 92

Figure 5.21 Complete glare control system ........................................................................... 94

Figure 5.22 Participants' selection of illuminance and color temperature for various activities . 97

Figure 5.23 Participants' selection of illuminance and color temperature for various activities

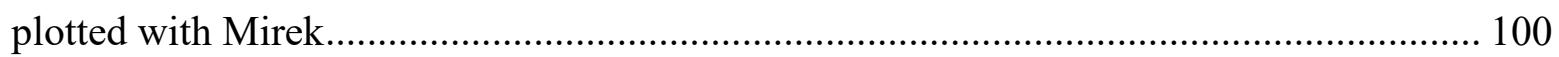

Figure 5.24 Proposed method of evaluating comfort using illuminance vs Mirek diagram........ 102

Figure 5.25 Chromaticity diagram of Philips hue A19 light bulb .......................................... 103

Figure 5.26 Placement of the three sensors and the pyramid suggested by Nazzal [7] .............. 106

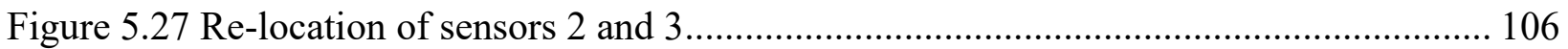

Figure 5.28 Suggested placement of light sensor pair in Bombardier Aerospace Global 7000

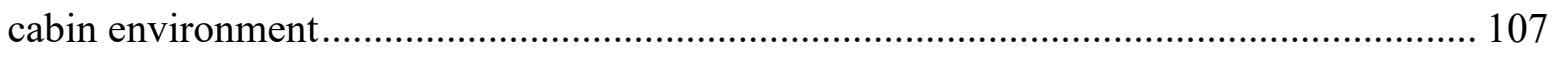

Figure 5.29 Effect of changing sensor angle from $0^{\circ}$ to $20^{\circ}$ and from distance of $45^{\prime \prime}$ to 70 " under same lighting conditions 108 
Figure 5.30 DGI vs distance at maximum brightness level and highest color temperature setting,

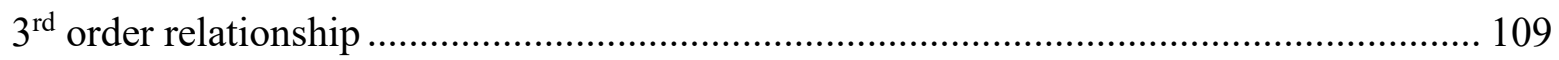

Figure 5.31 DGI vs distance at maximum brightness level and highest color temperature setting,

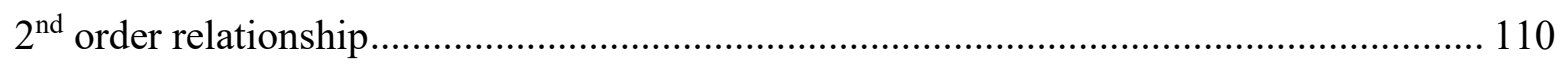

Figure 5.32 DGI vs distance at various brightness level and highest color temperature setting, $3^{\text {rd }}$

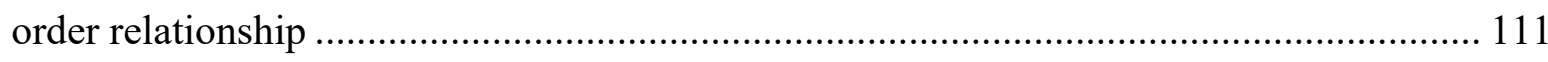

Figure 5.33 Linearly interpolated DGI vs DGI values obtained from estimation curves (left), and

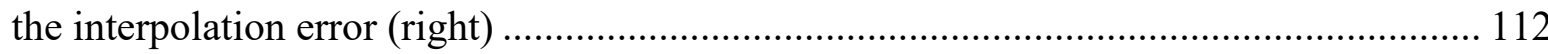




\section{Chapter 1 Introduction}

\subsection{Background}

Travelling by air is one of the most common ways to quickly get to the destinations of both long and short distances. With an already astounding 3.8 billion air travelers in 2016, it is expected that the number of air travel will be double by 2035 [1]. For such a large market that the aircraft industry foresees, a large number of low-cost airlines emerge to incite competition that becomes fiercer than ever. The aircraft companies are trying to differentiate themselves from others in various aspects. One of the most direct ways is to provide a better cabin environment.

Figure 1.1 shows a discomfort pyramid including various environmental components that can easily trigger passengers' discomfort feelings. Lighting condition plays the most important role in environmental comfort sensation. Assuming that usually there is no unpleasant odor.

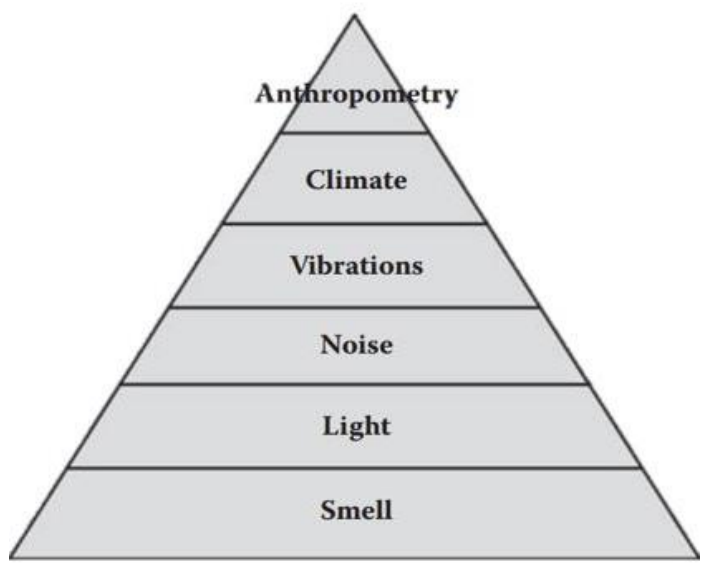

Figure 1.1 The discomfort pyramid [2]

There is a general consensus that we constantly adjust the lighting condition at home, in the office or classrooms for visual comfort. Good lighting conditions allow us to accomplish different tasks with required visual acuity. In most commercial aircrafts, cabin illumination relies heavily on cabin artificial lighting even during day time. The reason is that the high intensity of 
sunlight makes it impossible for naked eyes to look out of aircraft windows. Passengers will be left with no choice but to close the windows (Figure 1.2) to block sunlight coming into the aircraft. Prolonged visual discomfort could result in health-related problems such as headaches, eye sore, eye tiredness and deregulation of the circadian rhythm leading to depression [3].
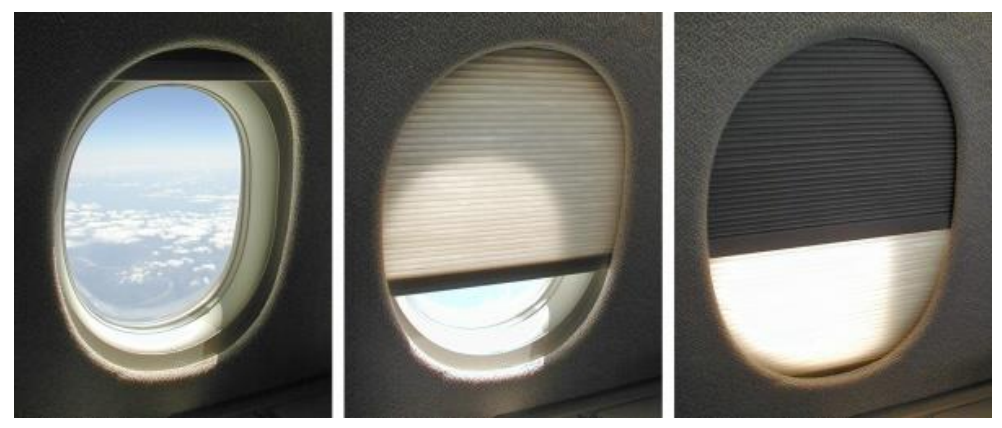

Figure 1.2 Mechanical window shades [4]

A solution to this inconvenience is to use smart windows which can allow variable amount of sunlight to come into aircraft cabin interior. Another advantage is to utilize a certain amount of sunlight for lighting up the cabin which can reduce the dependency of artificial lightings. There is a side benefit of using smart windows for airline crew members. With centralized control system, windows can be adjusted according to safety regulations without bothering passengers, especially during takeoff and landing when the accident rate is high [6].

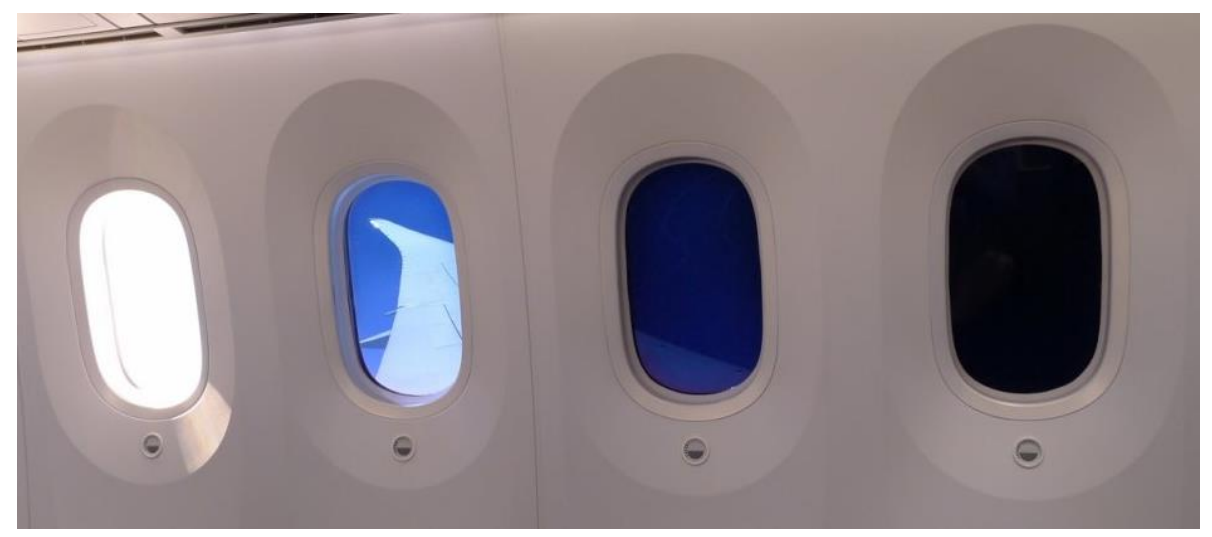

Figure 1.3 Electrochromic windows of various dimming levels [5] 
In use of smart windows, it is important to ensure that the dimming level of the window is sufficient to block enough sunlight so not to cause unpleasant visual feeling to passengers. In other words, there should not be any noticeable glare. With the idea of minimizing passenger discomfort in mind, if the dimming of windows can be done automatically, the passengers will not have to constantly worry about getting rid of the irritating sunlight, thus improve their travel experiences. In order to automate the dim level settings, it is necessary to quantify discomfort. A parameter to describe glare is to use daylight glare index DGI. The higher the DGI value, the more unpleasant the glare source is [7]. Therefore, the level of glare perceived by the passenger should be constantly monitored to adjust the window dim level accordingly.

Even though smart windows can allow a certain amount of external light into the cabin, there still will be situations where the utilization of sunlight is not possible. For example, when the sunlight is so strong, the window has to be completely dimmed, or at night no sunlight is available. In these situations, artificial lightings will be the dominant lighting source and their effects would be the primary judgment of visual comfort. However, since automated artificial illuminance control strategies have been proposed by various scholars, therefore, this aspect will not be repeated in this thesis. Rather an investigation of the relationship of illuminance and color temperature will be conducted, and the results can be used for controlling color temperature of artificial lighting when different illuminance values are outputted.

\subsection{Problem Statement and Objectives}

Smart windows are starting to replace traditional window shades on civilian aircrafts to improve passengers' flying experience due to the appealing advantage of the technology. However, a major concern arose as the inappropriate transparency of smart windows could actually lead to visual discomfort due to the bright sunlight. The current way to correct the 
problem is through manual operation, which may not be effective. Therefore, the objective of this thesis is to provide a method of eliminating visual discomfort via smart window transparency adjustment in an automated manner. The implementation issues are addressed in order to guarantee the practicality of the designed method. In addition, the elements regarding artificial lighting improving passenger visual comfort are investigated.

\subsection{Thesis Outline}

This thesis will be presented in the following order:

1. Introduction and Literature Review

2. Control of smart window

3. Discussion of implementation issues followed by the conclusion of work

This thesis begins by a literature review (Chapter 2). This chapter starts with the discussion of glare and the methods of glare detection. Then the knowledge of color temperature will be presented and lastly the lighting system control methods that scholars have attempted will be discussed.

In Chapter 3, the cabin mock up environment, experimental setup and the choice of equipment for the research work will be discussed.

In Chapter 4, the development of fuzzy logic control method for adjusting smart windows transparency with the utilization of glare index as discomfort indication will be presented.

In Chapter 5, the implementation of the control system and the issues of ensuring practicality of experimental setup on an aircraft cabin will be discussed. Also, the illuminance and color temperature about artificial lighting in aircraft cabin will be investigated. 
Chapter 6 concludes this thesis by summarizing the results and findings. The contributions of the thesis will be presented along with the suggestions for future work. 


\section{Chapter 2 Literature Review}

A literature review was conducted to gain a fundamental understanding of the state of art research for glare evaluation, fuzzy logic lighting system control methods, and the effect of color temperature of artificial lighting on visual satisfaction. Although the topic regarding visual comfort has been investigated for many years, the recent research on visual comfort/discomfort still relies on previously established concepts and results. Therefore, these fundamental concepts and results are reviewed along with the inclusion of newer research results.

\subsection{Glare Evaluation Methods}

The evaluation of perception of lighting to human eye in an objective manner is difficult since visual comfort is usually subjective to feeling and the degree of comfort can be very extensive. Therefore, instead of evaluating comfort directly, researchers have been focusing on defining discomfort which could be readily defined using glare indices. According to The Lighting Handbook published by the Illuminating Engineering Society of North America (IESNA) [8], glare is the sensation produced by luminance within the visual field that is sufficiently greater than the luminance to which the eyes are adapted to. Therefore, glare will cause pain, discomfort or loss in visual performance and visibility. There are two types of glare: disability glare and discomfort glare.

Disability glare is associated with the reduction invisibility due to scattered light in eye [9]. At certain viewing angles, the contrast of details becomes lower causing things difficult to see. It is possible to quantify disability glare by comparing the visibility of an object seen in the presence of the glare source with the visibility of the same object seen through a uniform luminous veil. However, disability glare does not cause physical discomfort feeling. 
The other type of glare is discomfort glare, which is a discomfort or painful sensation when exposed to a bright light in the field of view. However, it does not cause vision impairment or reduction in visual performance [9]. Therefore, it is desirable to eliminate discomfort glare while ensuring a good level of illumination for a given task. Cédric et al. [10] suggested that luminaires should be designed to contribute to natural light and then to provide a suitable level of illumination. In other words, a daylight source should be utilized as much as possible before using artificial lighting.

In case that the discomfort glare source is from window, Piccolo and Simone [11] found that the magnitude of discomfort glare loosely depends on dimension and distance from the observer, but strongly depends on the brightness of the sky portion visible from the window. On mild degrees of glare, observers seem to exhibit greater tolerance to glare effects originating from daylight than to those originating from a comparable artificial lighting situation. This finding gives confidence that the results of research performed indoor using artificial lighting would be sufficient or comparable to the system implemented outdoor.

Glare measurements can be reflected by glare index. However, there is no standardized evaluation method as each index was proven sufficient only for certain scenarios. Fundamentally, these indices consist of four basic factors: 1) luminance of glare source, 2) angular subtence of glare source at eye, 3) luminance of background, and 4) deviation of glare source from line of sight [12]. The early version of glare evaluation posed the issue with evaluating glare at high luminance levels or when the sources were large. Even with the use of corrective factors, no satisfactory results could be obtained [13]. It was suggested that when perceiving discomfort glare from small electric light sources, the observer's adaptation level was virtually independent of the luminance of small glare sources. The equations for such assessments are only valid over 
the range where the adaptation level is determined primarily by the background luminance [12], indicating that the physical dimensions of light sources chosen for glare experiment should be comparable to the dimension of the actual glare source.

Unified glare index (UGR) and daylight glare index (DGI) are the two glare indices developed more suitable for indoor scenarios than other indices such as glare rating GR [9]. UGR is able to describe the combined effect of luminance, size and location of the luminous environment of the room. But the potential glare source contribution to an observer's adaptation, and the direct illuminance at the eye are omitted [12]. Some argued that such effect is negligible when artificial lighting is of dominant concern [12]. On the other hand, UGR is known to be accurate only for small source sizes such as standard incandescent bulb [9]. Regardless, due to the simpler calculation procedure, UGR has gained its popularity [12].

DGI was modified from the Cornell Formula by taking into account of daylight luminance as an additional parameter [13]. The original DGI provided an overestimated measure of glare sensation from a wider source [14]. To overcome this problem, Nazzal [7] introduced $\mathrm{DGI}_{\mathrm{N}}$ (equation 1) by considering the direct sunlight component and replacing the background luminance with the adaptation luminance $L_{\text {adaptation }}$ for the purpose of including the contribution of the immediate surrounding luminance of the source and the surroundings.

Since all glare indices involve the four basic factors, the selection depends on the scenario of the glare sources under this study. With direct luminance at the eye and daylight as a major source of glare, $\mathrm{DGI}_{\mathrm{N}}$ is the most suitable glare evaluation method for the cabin environment under this research. $\mathrm{DGI}_{\mathrm{N}}$ is expressed as: 


$$
D G I_{N}=8 \log _{10}\left\{0.25\left\{\frac{\sum\left(L_{\text {exterior }}^{2} \Omega_{p}\right)}{L_{\text {adaptation }}+0.07\left(\sum\left(L_{\text {window }}^{2} \omega\right)^{0.5}\right)}\right\}\right\}
$$

where $\omega(\mathrm{sr})$ is the solid angle subtended by the window to the point of observation, $\Omega_{p}(\mathrm{sr})$ is the solid angular subtence of the source modified for the effect of the position of its elements in different parts of the field of view, $L_{\text {window }}\left(\mathrm{cdm}^{-2}\right)$ is the average vertical luminance of the window that is calculated from the reading of the sensor with a shielding pyramid, and $L_{\text {exterior }}\left(\mathrm{cdm}^{-2}\right)$ is the average vertical unshielded luminance of the outdoor. The model is rendered as a $\log$ function to emphasize that human eye perception to light/glare is non-linear [10].

To evaluate the effect of glare sensation, a set of DGI values are listed in Table 2.1. This is the Hopkinson's scale which was adopted in multiple papers [11], [15] and [16]. Paper [11] and [16] were cited 55 and 53 times respectively. The high number of citations gives confidence that the DGI can be accepted as the indication of discomfort. It should be noted that in Table 2 "negligible" merely means that the amount of light that results in discomfort glare is negligible to human eyes.

Although can be represented in terms of various indices, discomfort glare still remains a subjective feeling. Each person has different tolerances and preferences. For example, Huang et al. [13] mentioned that Japanese were generally more tolerant to glare than Americans. The use of the Hopkinson's scale only provides a likelihood of discomfort.

Another source of light that might come into discomfort picture is a digital device monitor, which is often referred to as the entertainment system on aircraft. Study suggested that ambient illuminance has no or insignificant effect on visual comfort regardless of age [13]. The 
other authors conducted some experiments only under low illuminance environment (between 50lux to 1200lux). Table 2.2 and Table 2.3 show various outdoor and indoor lighting scenarios respectively from paper [17] and [18].

Table 2.1 DGI value scale [11]

\begin{tabular}{|c|c|}
\hline DGI & Perceived Glare \\
\hline$<16$ & Negligible \\
\hline 16 & Just perceptible \\
\hline 18 & Noticeable \\
\hline 22 & Acceptable \\
\hline 24 & Just uncomfortable \\
\hline 26 & Uncomfortable \\
\hline 28 & Just intolerable \\
\hline 29 & Intolerable \\
\hline
\end{tabular}

Table 2.2 Illuminance values (lux) at various outdoor scenarios [17]

\begin{tabular}{|c|c|c|c|}
\hline Scenario & Lux & Scenario & Lux \\
\hline Direct sunlight & $10,000-100,000$ & Dark night & $10-100$ \\
\hline Dark day & $100-1,000$ & Twilight & $1-10$ \\
\hline Full moon & 0.1 & Starlight & 0.001 \\
\hline
\end{tabular}

Table 2.3 Illuminance values (lux) at various indoor scenarios [18]

\begin{tabular}{|c|c|c|c|c|c|}
\hline Scenario & Lux & Scenario & Lux & Scenario & Lux \\
\hline Parking lots & 50 & Corridor & $100-150$ & Dining rooms & 200 \\
\hline Library & 300 & Offices & $400-500$ & Art offices & 750 \\
\hline Electronics assembly & 1,000 & Hand tailoring room & 2,000 & & \\
\hline
\end{tabular}

From these Tables it may be noticed that it is not necessary to have ambient illuminance that exceeds 750 lux in an aircraft cabin. Therefore, in this study the device monitor is not treated as a glare source. 
Piccolo and Simone [11] attempted to reduce the glare effect throughout the day by using electrochromic windows and by maintaining the DGI level at 22 (Figure 2.1) which is considered as the maximum value for an office setting. However, it was mentioned that such results were successful only for high altitude where no high luminance spot appearing on the window. This reveals the problem of using spot sensors and glare indices as the main method of glare detection. If assuming that the luminance level throughout the entire window panel is constant, sensors will not be able to recognize the areas with abnormally high luminance such as the sun. Thus, the actual effect of the concentrated light spot to the eye may be underestimated.

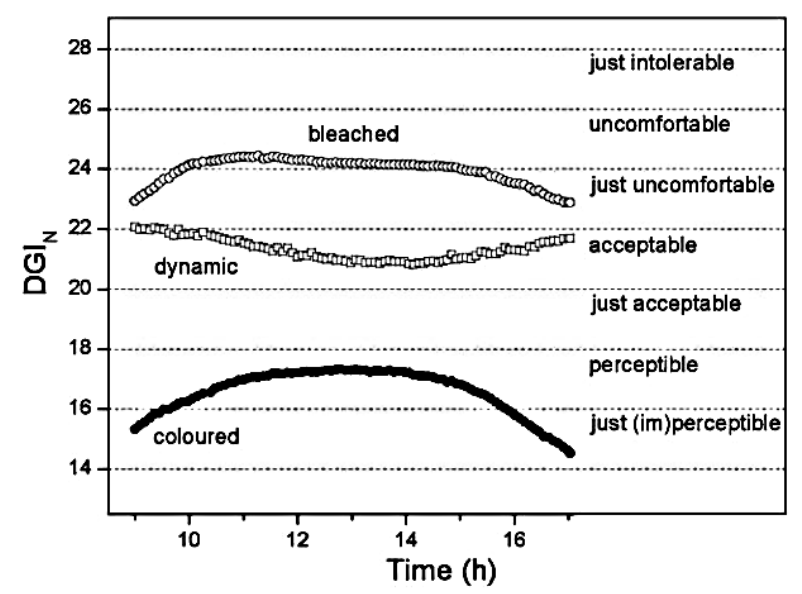

Figure 2.1 Glare reduction result by Piccolo [11]

In spite of the weakness of using sensors to detect glare, the method that Fernandes et al. [19] suggested proved to be a feasible solution. This method was to detect a luminance value byy taking fisheye view pictures of the surrounding. The camera view angle must be wide enough to cover the light source including the window and the working area. After the picture was taken, it was converted into a $10 \times 10$ pixelated image as shown in Figure 2.2. Each pixel represented the average luminance of a small area. 


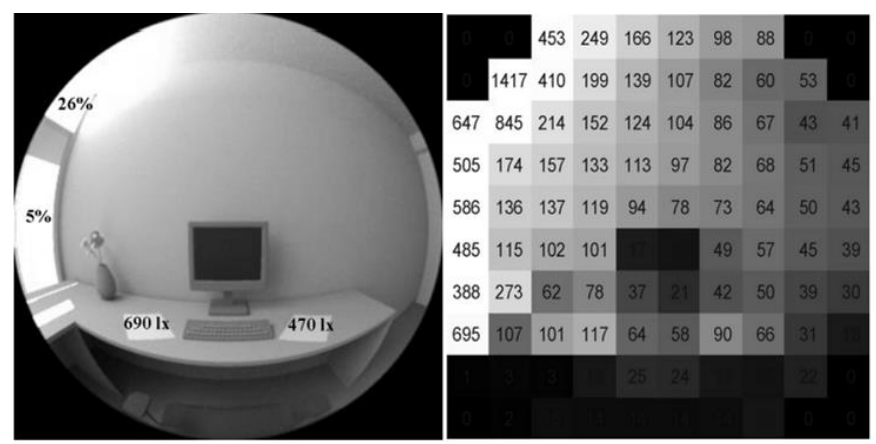

Figure 2.2 Original picture (left) and pixelated picture with luminance values (right) [19]

From the pixelated image, the left-most column represents the luminance of the window, ranging from $388 \mathrm{~cd} / \mathrm{m}^{2}$ to $685 \mathrm{~cd} / \mathrm{m}^{2}$. For a large window, if spot sensors were used, then possibly the average luminance of $551 \mathrm{~cd} / \mathrm{m}^{2}$ would be detected, which resulted in approximately $100 \mathrm{~cd} / \mathrm{m}^{2}$ difference between the highest and lowest values.

Leclercq et al. [20] also used a fisheye lens camera to take a picture of the office area and performed analysis on the picture. Instead of calculating the respective average luminance of each pixel, the high contrasts zones were identified and compared to the background luminance. The number of high contrast zones along with the mean illumination of the office area was related to the comfort level of their own designed scale using fuzzy logic to decide the optimum state of the window blinds

Several researchers including Cédric et al. [10], Wienold and Christoffersen [21], and Suk and Schiler [22], used comparable camera methods for evaluating visual discomfort. One common characteristic is that their subject areas were generally large and spacious so that the occupants tended to be affected by a number of glare sources or luminaires. This suggests that in scenarios with large and multiple glare sources, analyzing pictures to evaluate the overall lighting environment is desirable. 

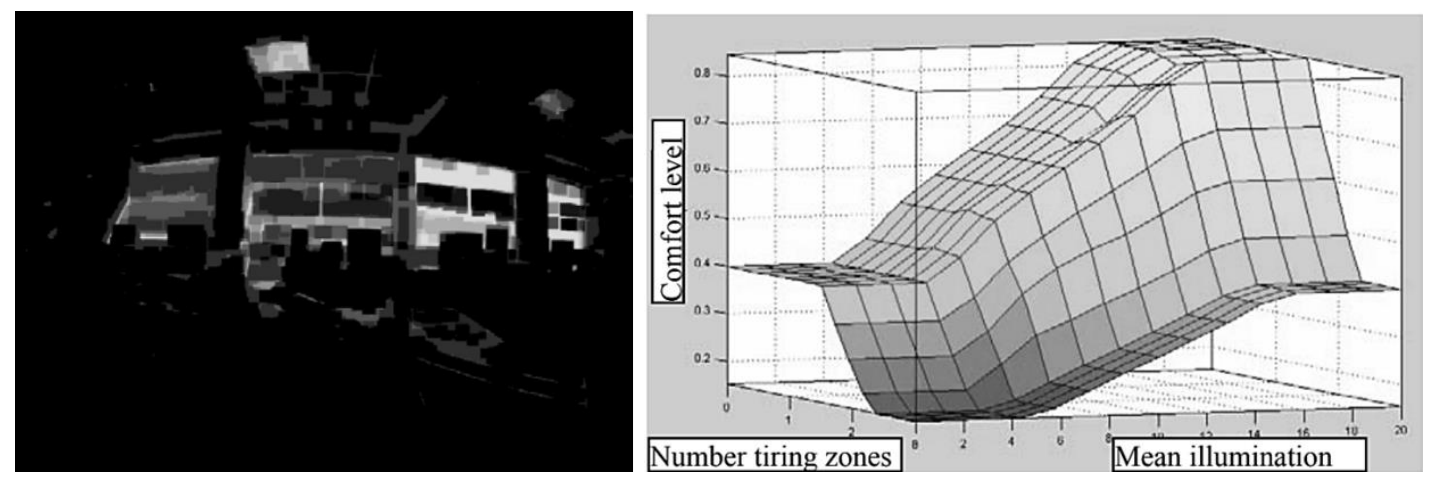

Figure 2.3 Processed image for finding high contrast zone (left), fuzzy logic for evaluating comfort level (right) [20]

In contrast, in enclosed areas such as aircraft cabin, the glare source that the passengers encounter will only come from the windows that are generally small in size. The method of pixelating images might be unnecessarily complex and time consuming. Also, in office areas, the lighting environments are relatively stable compared to the lighting environment in the aircraft cabin that is forever changing. High responsiveness and quick real-time glare detection is required for aircraft cabin.

Lastly, scholars have provided some basic information through their work. Piccolo and Simone [11] found that the excessive daylight levels in interiors do not always correspond to optimum visual conditions as they could produce an excessive luminance range in the visual field with high risk of unwanted glare effects. In terms of luminance values, direct sun luminance is generally higher than $10^{6} \mathrm{~cd} \mathrm{~m}^{-2}$ while average sky luminance can vary from $2000 \mathrm{~cd} \mathrm{~m}^{-2}$ to $8000 \mathrm{~cd} \mathrm{~m}^{-2}$. Also, IESNA [23] suggests the maximum allowable luminance levels in interiors recommended by lighting standards under artificial lighting are $500 \mathrm{~cd} \mathrm{~m}^{-2}$ in the center of the visual field and $2000 \mathrm{~cd} \mathrm{~m}^{-2}$ outside the normal visual field. However, these values could be doubled if under daylight illumination [24]. 


\subsection{Smart Windows}

Traditional blinds, curtains, shades etc. often reduce glare at the expense of useful daylight so that artificial lightings have to be used in the room despite high external light availability [11]. Smart windows provide a solution of utilizing the external lighting to various adjustable extents such that the amount of light entering the area of concern is reduced to lower or eliminate discomfort to occupants. There are four types of electrically controllable smart windows: electrochromic EC, polymer dispersed liquid crystal PDLC, suspended particle SusP and micro-blind. Table 2.4 shows only the properties of three different types of smart windows, since there is not much information about new development of micro-blinds. The properties depend on the manufacturers as well as the generation of products through development phases. But the information in Table 2.4 provides a general idea of the performance comparison between the three common smart windows.

PDLC and SusP windows share a similar principle of operation. When electric field is applied to the window, the particles in the substrate layer align up so that light can pass through the gap. The difference of the two is the alignment method. In a SusP device (Figure 2.4), when electric field is absent, the particles suspend randomly in the substrate therefore blocking off light passing through the window. When electric field is applied across the conductive coatings, the particles align up in parallel to the direction of electric field therefore allowing light to pass through the gaps of each row of particles, turning the window clear. 
Table 2.4 Properties of three different types of smart windows

\begin{tabular}{|c|c|c|c|}
\hline & $\begin{array}{c}\text { Polymer Dispersed } \\
\text { Liquid Crystal } \\
\text { (PDLC) }\end{array}$ & Electrochromic (EC) & $\begin{array}{c}\text { Suspended Particle } \\
\text { (SusP) }\end{array}$ \\
\hline Powered off & Tinted white & Clear, greenish-yellow & $\begin{array}{c}\text { Tinted ark blue or } \\
\text { black }\end{array}$ \\
\hline Powered on & Clear (slight haze) & Dark Blue & Light Blue \\
\hline Variable State & Yes & Yes & Yes \\
\hline Memory & No & Yes (for few minutes) & No \\
\hline Power & $\sim 120 \mathrm{VAC}$ & $\sim 1 \mathrm{VDC}$ & 35 to $100 \mathrm{VAC}$ \\
\hline Response time & $\sim 0.1 \mathrm{~s}$ on, $\sim 1 \mathrm{~s}$ off & $\sim 90 \mathrm{~s}$ on, $\sim 200 \mathrm{~s}$ off & $\sim 3 \mathrm{~s}$ on, $\sim 3 \mathrm{~s}$ off \\
\hline $\begin{array}{c}\text { Light admissibility } \\
\text { when tinted }\end{array}$ & $4 \%$ to $9 \%$ & $\sim 0.001 \%$ to $0.1 \%$ & $0.4 \%$ to $5 \%$ \\
\hline $\begin{array}{c}\text { Light } \\
\text { admissibilitywhen } \\
\text { clear }\end{array}$ & $45^{\circ} \%$ to $81^{\circ} \%$ & $\sim 70 \%$ & $48 \%$ to $57^{\circ} \%$ \\
\hline Temperature & $-13^{\circ} \mathrm{F}$ to $186^{\circ} \mathrm{F}$ & $14^{\circ} \mathrm{F}$ to $204^{\circ} \mathrm{F}$ & $-22^{\circ} \mathrm{F}$ to $194^{\circ} \mathrm{F}$ \\
\hline $\begin{array}{c}\text { Curved Design } \\
\text { Possible? }\end{array}$ & Yes & Yes & Yes \\
\hline $\begin{array}{c}\text { Cabin Management } \\
\text { System Integration }\end{array}$ & Yes & Yes & Yes \\
\hline $\begin{array}{c}\text { Crew Override } \\
\text { Yes }\end{array}$ & Yes & Yes \\
\hline
\end{tabular}

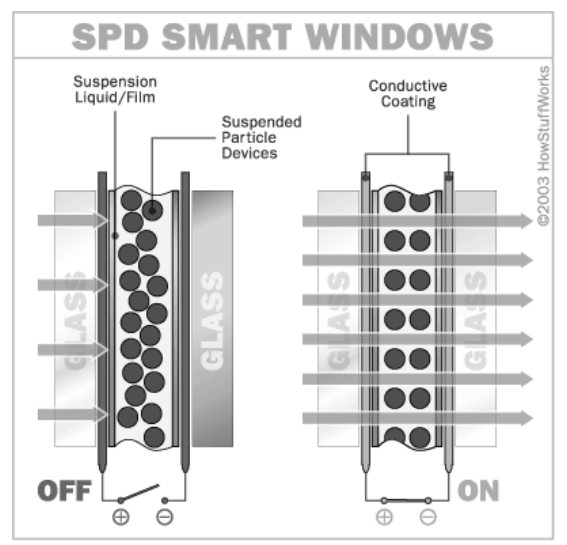

Figure 2.4 Illustration of the operation of a suspended particle device [25] 
In PDLC device (Figure 2.5), on the surface of each randomly orientated particle carries, a director reflects light in different directions to scatter across the entire film. When an electric field is applied, the directors will orientate in parallel to the electric field allowing light to pass through the gaps between adjacent directors [26]. This light scattering mechanism explains why even during the most tinted state, the PDLC window will still have high light admissibility. Therefore, it is not applicable for light blocking light, rather for indoor office areas for privacy purpose.

(a) Off state

(b) On state

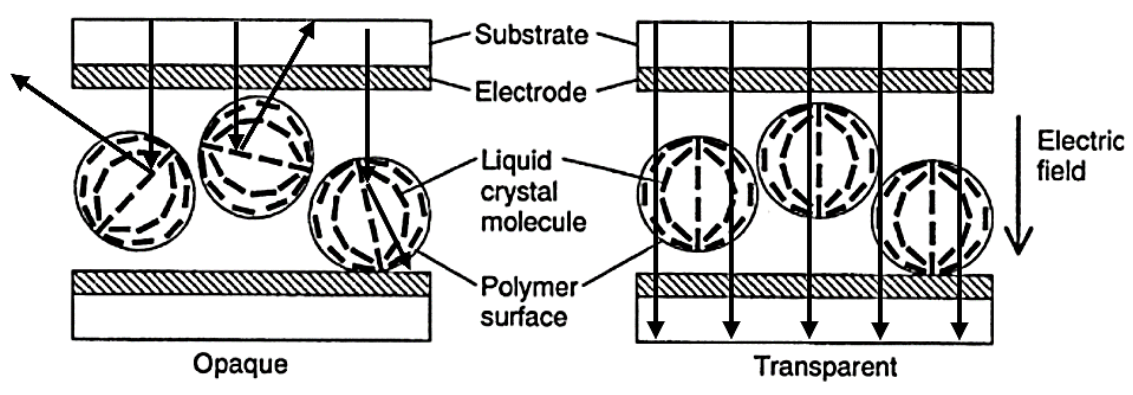

Figure 2.5 Illustration of the operation of a polymer dispersed liquid crystal device [26]

EC windows, on the other hand, do not operate through the alignment of particles. At the two layers of glass that sandwich the electrochromic substrate, there are two electrodes that allow an oxidation reaction to take place between the two compounds in the substrate (Figure 2.6). The resultant compounds from the chemical reaction change in properties to absorb light of certain wavelengths, thereby turning the window opaque.

By understanding the different types of smart windows, SusP and EC windows seem to be the two feasible options for reducing glare. EC window is deemed the best option for the cabin application considering the lower light admissibility at the opaque state and the low power required. 


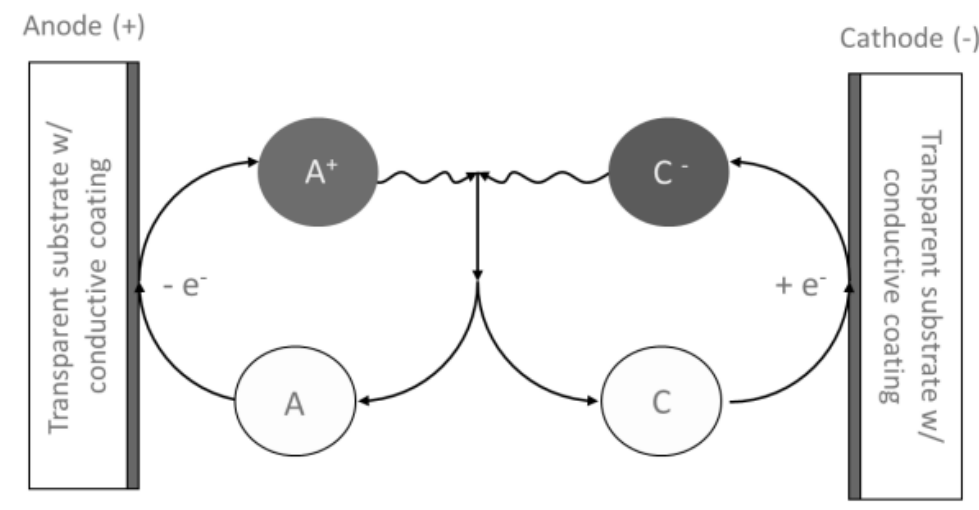

Figure 2.6 Illustration of the operation of an electrochromic window (provided by Gentex)

The downside of EC window is the long transition time compared to the other two windows. Newer generation of EC windows have been improved to have a faster transition time. Nevertheless, from the user's point of view, one might not actually appreciate the very fast transition time from the window if it was to operate automatically. Any change that is too sudden might shock aircraft passengers. Therefore, the long transition time of electrochromic window might also serve as an advantage.

Little research has been done to evaluate the practicality, energy consumption and glare reduction issues of using EC window. In research done by Lee and Travil [27], they set up a test environment which replaced the entire window panel by EC window. the authors emphasized that even though the test environment was installed overhanging, they did not block the direct orb of the Sun. The combination of EC window and Venetian blinds was recommended over the sole use of EC window unless the occupants could reposition. In terms of energy savings, the authors found that the saving was minimal for small windows. However, the glare evaluation results suggested that the DGI level can always be maintained below the border line of level 22 . This brings to the idea that energy consumption and visual comfort is a trade-off matter. 
Another research was done by Bisegna [28]. An EC window was analyzed with a linear strategy to change the tinting state gradually to increase solar total irradiation. The author also mentioned the attempt to trigger the transparency of EC window based on the outdoor temperature. However, solar radiation does not necessarily correspond to temperature. This could lead to windows being tinted while the external environment is dark. This suggests that there is no direct way better than using direct light sensing method to evaluate visual comfort. The author developed two different systems to utilize EC window. One was visual comfort based and the other was energy based. In terms of comfort based system, it was suggested that glare could be eliminated and indoor illuminance could be maintained at the desired level but no energy saving was observed. For the energy based system, the results demonstrated that the functionality of EC windows were minimal that it made no difference from using clear glass windows. Piccolo and Simone [11] and Fernandeset al. [19] both arrived at a similar conclusion. Ochoa et al. [3] attempted to optimize the two factors using a façade system but eventually concluded that the optimization criteria could hardly be met. Therefore, the only advantage of using EC window is for visual comfort.

\subsection{Effect of Color and Color Temperature on Visual Comfort}

When light enters human eye, there are two classes of receptors to perceive the light before transmitting the signal into the brain, they are the rods and cones. Cones are responsible for color vision and also brighter environment, while rods are responsible for night vision. The corresponding visions are called photopic vision $V(\lambda)$ and scotopic vision $V^{\prime}(\lambda)$ respectively. It is evident that rod receptors are much more sensitive to light than cones [29]. Under photopic condition, human perceives wavelength of $550 \mathrm{~nm}$ the brightest while that of $360 \mathrm{~nm}$ and $800 \mathrm{~nm}$ 
will be the dimmest (Figure 2.7). Table 9 shows the boundaries of various colors as a generally accepted wavelength of each color.

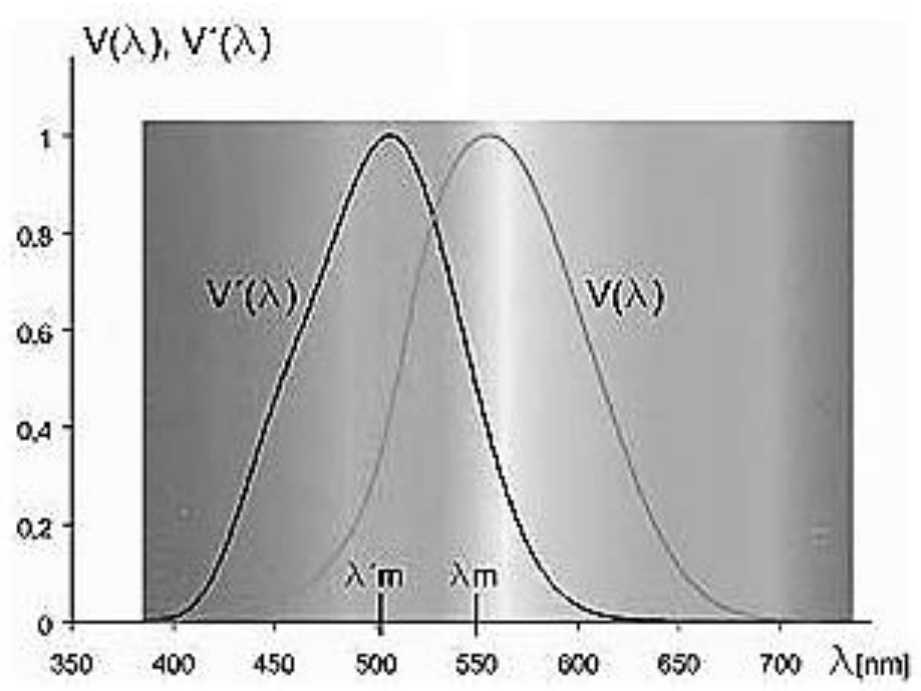

Figure 2.7 Photopic and scoptopic vision response curves [30]

Table 2.5 Wavelengths of various colors

\begin{tabular}{|c|c|c|c|c|c|}
\hline Purple & Blue & Green & Yellow & Orange & Red \\
\hline $360-450 \mathrm{~nm}$ & $450-500 \mathrm{~nm}$ & $500-570 \mathrm{~nm}$ & $570-590 \mathrm{~nm}$ & $590-610 \mathrm{~nm}$ & $610-800 \mathrm{~nm}$ \\
\hline
\end{tabular}

Itten [31] suggested the lighting color conditions does affect the perceived thermal comfort, which is in correspondence to the main stream studies that suggest color affects emotions, performance and other related psychological issues. Winzen et al. [32] has conducted a research in this area that is specifically tailored for aircraft passenger thermal comfort with red, yellow, orange, blue, green and violet colors controlled by LEDs. With 59 participants in the research, it was found that yellow light would lead them to perceive the environment as warmer in terms of temperature, while blue light would be perceived as cooler. On the other hand, light with blue hue was perceived as being brighter and light with yellow hue was perceived as darker, which led to an increase of alertness in blue light and in yellow light, participants felt sleepier. 
Blue light has been a special topic. It was suggested that when human is exposed to light with large portion of blue wavelengths could lead to melatonin suppression, a hormone that is released by the brain's pineal gland that directly affect sleep quality [33]. However, this was concluded with luminous blue colored light, for a non-luminous blue color, Valdex and Mehrabian [34] suggested that such color would actually lead a smoothing effect.

Color temperatures on the other hand, characterize the spectral properties of a near-white light source, it is measured in degrees Kelvin $(\mathrm{K})$ to which one would have to heat a blackbody radiator to produce light of a particular color [35]. Table 2.6 shows the color temperatures of various illuminants, the higher the color temperature the "warmer" it is, and the lower the color temperature the "cooler" it is. Human eyes are more sensitive to the color temperatures ranges from $2000 \mathrm{~K}$ to $6000 \mathrm{~K}[36]$.

Table 2.6 Color temperatures of various light sources [35]

\begin{tabular}{|l|c|}
\hline Light Source & Color Temperature (K) \\
\hline Candle flame & 1900 \\
\hline Sunlight at sunset & 2000 \\
\hline Tungsten bulb (60W) & 2800 \\
\hline Halogen lamp & 3300 \\
\hline Overcast sky & 6500 \\
\hline
\end{tabular}

It is well known nowadays that illuminance and color temperature are the important factors for us to perform various tasks. Sinoo et al. [37] investigated the lighting condition at a nursing home, at which the target subjects were elderly residents. In addition to the mean illumination considerations, strong emphasis was put on color temperatures. Recommendations that combined the two factors were made to improve safety, visual comfort and lighting related health problems such as insomnia, in view of the possibility that biological clock could be 
affected by improper lighting that results in depreciating sleep quality. On the other hand, the authors mentioned that sudden change of high illumination area to low color temperature and low illumination area would result in visual adaptation issues that are significant for elderly.

Other than safety concerns, in general, people prefer some specific color temperatures for different illuminance levels, such correlation originated from the Kruithof's curve which was developed by Kruithof in 1941 [38].

Kruithof's curve (Figure 2.8) suggests that the area that a person feels comfortable to have the respective color temperature at various illuminance level is very narrow, as that represented by the white area, the grey area represents the area where people feel uncomfortable. However, Kruithof's work was critically criticized because of the lack of test subjects and technical explanations.

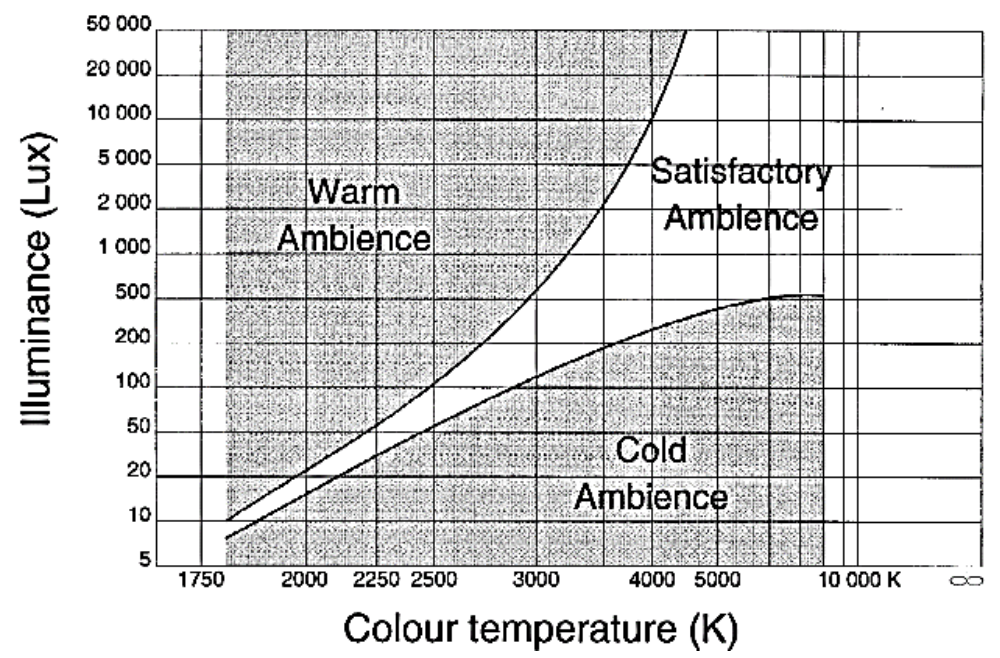

Figure 2.8 The Kruithof's curve [36]

The concept of his work however, was widely referenced by multiple scholars such as [36], [37],[39] and [40] as certain standard for lighting condition evaluation or application, and it also sparked the concept of correlating the two parameters. This might mainly because of the 
lack of even better graphical and simpler correlation of the two parameters. Boyce and Cuttle [41] did however suggested that color temperature of lighting had no correlation with illuminance, but Bodman [42] produced the following table suggesting the subjects' perception of comfort under various color temperature and illuminance conditions. It was concluded in low illuminance environment, warm white was unpleasant while high color temperature was regarded as "cool" which means it was also not preferable, while with high illuminance, warm white was regarded as artificial. Also, at the mid illuminance level, it seemed that all color temperatures were acceptable.

Table 2.7 Perception of various color temperatures concluded by Bodman [42]

\begin{tabular}{|c|c|c|c|}
\hline Illuminance (lux) & Warm white & White & Daylight \\
\hline$<700$ & Unpleasant & Dim & Cool \\
\hline $700-3000$ & Pleasant & Pleasant & Neutral \\
\hline$>3000$ & Excessive, artificial & Pleasant, lively & Pleasant \\
\hline
\end{tabular}

Even with the conclusion of the work from the few scholars on color temperatures, plenty of research were conducted afterwards with various environment considerations. For example, Oiand Takahashi [43] conducted another study that extended the preference of color temperature towards various settings - living room, dining room and bedroom, and the results that they obtained is listed in Table 2.8.

Table 2.8 Preferred illuminance levels and color temperatures for various activity settings [43]

\begin{tabular}{|l|l|l|}
\hline Setting & Illuminance level Preference & Color Temperature Preference \\
\hline Retiring space & Low & Very warm white \\
\hline Bedroom & Low & Warm white \\
\hline Dining & Medium to High & Warm white to white \\
\hline Studying & High & White to daylight \\
\hline
\end{tabular}


The general idea was that the higher the concentration was required for the activity, the higher the color temperature was preferred and for relaxing, low to very low color temperatures were more preferable.

\subsection{Use of Fuzzy Logic in Lighting Systems}

The glare evaluation method was discussed in section 2.1. It was suggested that light sensors are more preferable and in fact many researches have used light sensors for direct illuminance and glare evaluation. The detected values would then be passed on to their respective lighting and/or blind control system for illuminance control. Plenty of researches have already put focus on how to maintain a consistent vertical and/or horizontal illuminance level. Many of them have used fuzzy logic to implement the system given that the attempts of modeling of illumination with the inclusion of color temperature has not been yielding results that can be applied for large variety of scenarios [36].

Saravanan [44] suggested using two fuzzy sets to control the luminance of a light bulb through a parallel resistor circuit that responded to the environmental lighting. The two inputs were "environmental light" and "change rate of environmental light", each with 3 membership functions and the output control consist of 6 membership functions (see Figure 2.9).

Figure 2.10 shows the bulb response under various environmental lighting. The benefit of using an extra input to consider rate allowed the system to respond quicker to sudden change in environmental lighting compared to a SISO system. The similar system can also be applied to fluorescent lamp that responds to external lighting [45]. 

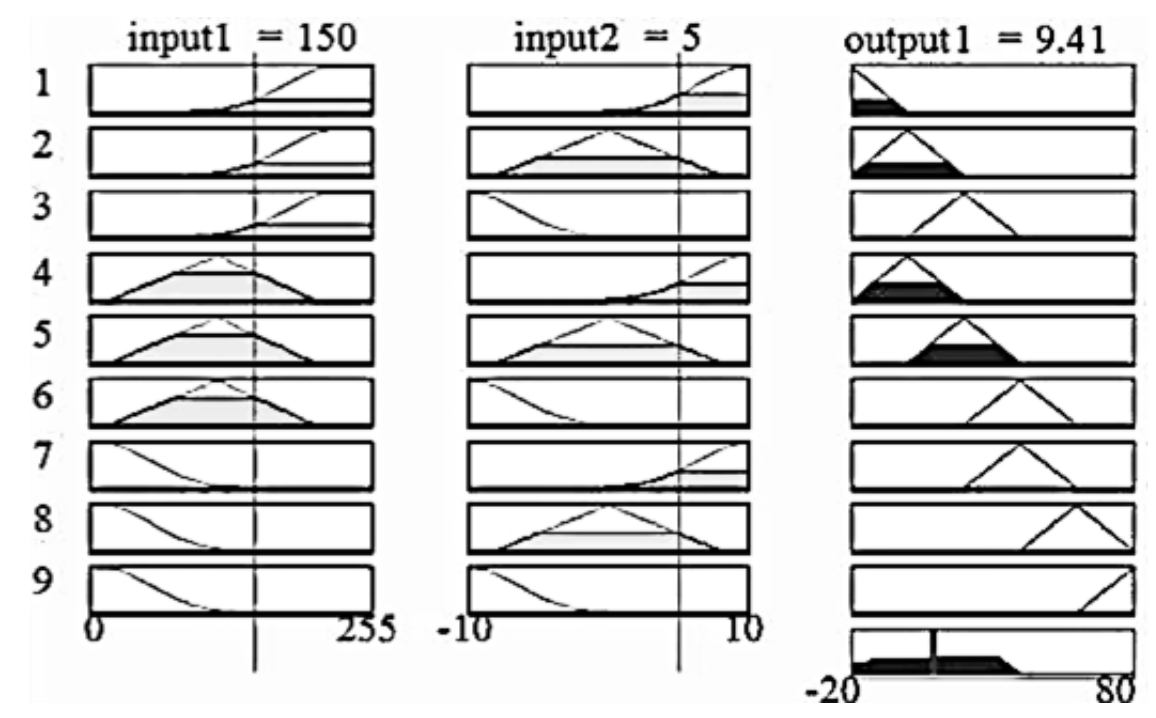

Figure 2.9 DISO fuzzy logic system proposed by Saravanan [44]

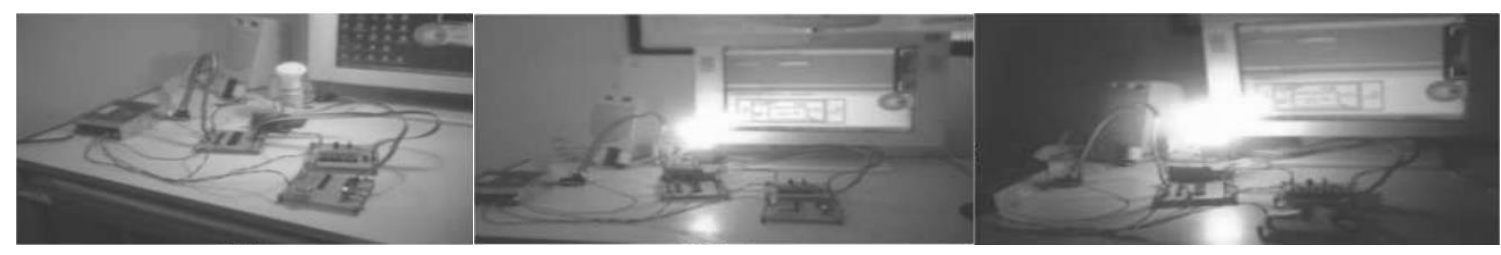

Figure 2.10 Response of light bulb under bright (left), moderate (center) and dark (right) environment [44]

Kolokotsaet al. [46] implemented fuzzy logic for controlling lighting system, thermal control and $\mathrm{CO}_{2}$ concentration control. Instead of using fuzzy logic for direct output, the authors demonstrated the use of a few different types of controllers - fuzzy PID controller, fuzzy PD controller and adaptive fuzzy PD controller. All the controllers were able to yield acceptable results to maintain the parameters at the respective set points. The research started with the demonstration of a fuzzy PID controller, which consisted of a PI part summed with a D part, to yield the output, but it was then suggested that fuzzy PD controller was better in a way that it could yield the same results with simpler architecture, less rules and required less computation time. And when adaptive fuzzy PD controller was compared to a non-adaptive one, the former could eliminate overshoot, better maintain the system at the set point and more importantly 
reduced the overall energy consumption when controlling thermal comfort (Figure 2.11). However, in terms of illumination, there was no need for an adaptive controller since the nonadaptive version already yielded optimum response that fulfilled user preferences of illumination.
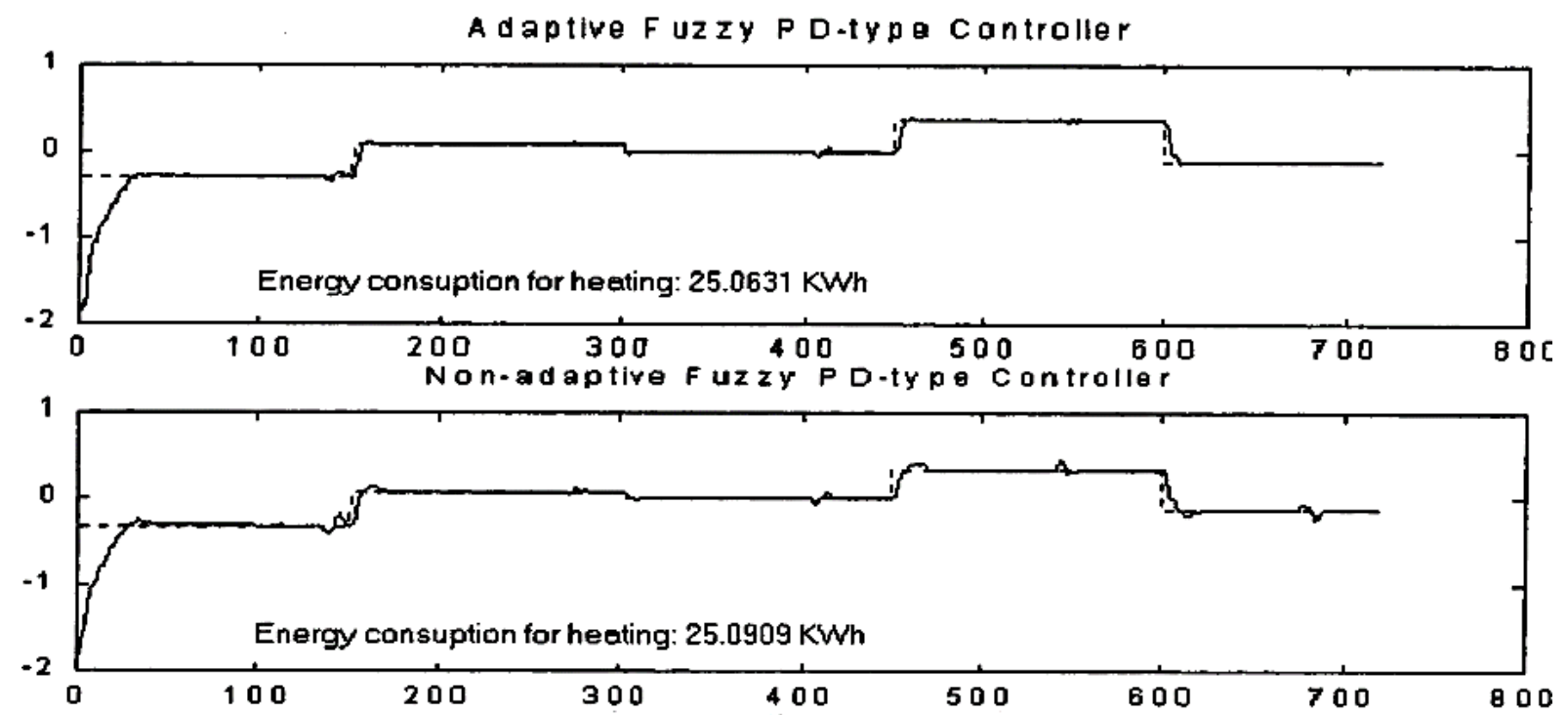

Figure 2.11 Comparison of thermal control results using adaptive (top) and non-adaptive (bottom) fuzzy PD controller [46]

Kolokotsa et al. [46] included user preference in the control system, but glare was not one of the comfort parameters, and the illumination level information that gathered from each user was used as the indication. As long as the control system could maintain the set point, then visual comfort was concluded to be successful. Research in [47] and [48] had a different definition of user preference. It was modeled by utility function (Figure 2.12) that changed over time and also with the task the occupants were performing. It represented the degree of comfort at various illumination levels and it was expected to provide promising results for using theoretic decision techniques for reliable utility elicitation [49]. Combined with the comfortable illumination values, that were obtained by fuzzy logic from daylight and artificial lighting contrition, and with motion sensing to detect the location of user, the required illumination value was calculated and the error 
was used as the input for a PI controller (Figure 2.13). However, the author also noticed that it was impractical to expect users to continuously provide their preference for system input.

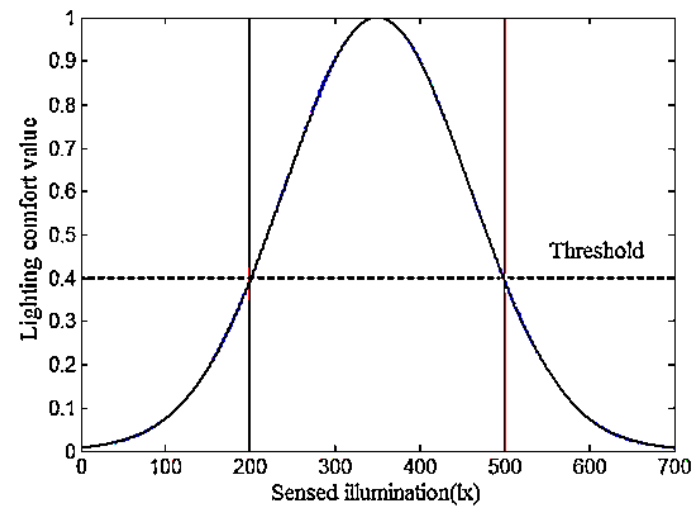

Figure 2.12 Graphical representation of utility function [49]

$$
\text { utility function }=\left\{\begin{array}{c}
\exp \left(\frac{-(x-\mu)^{2}}{2 \sigma^{2}}\right), \text { if } x \in[\mu \pm \sigma \sqrt{-2 \ln (t)}] \\
\text { 0, otherwise }
\end{array}\right.
$$

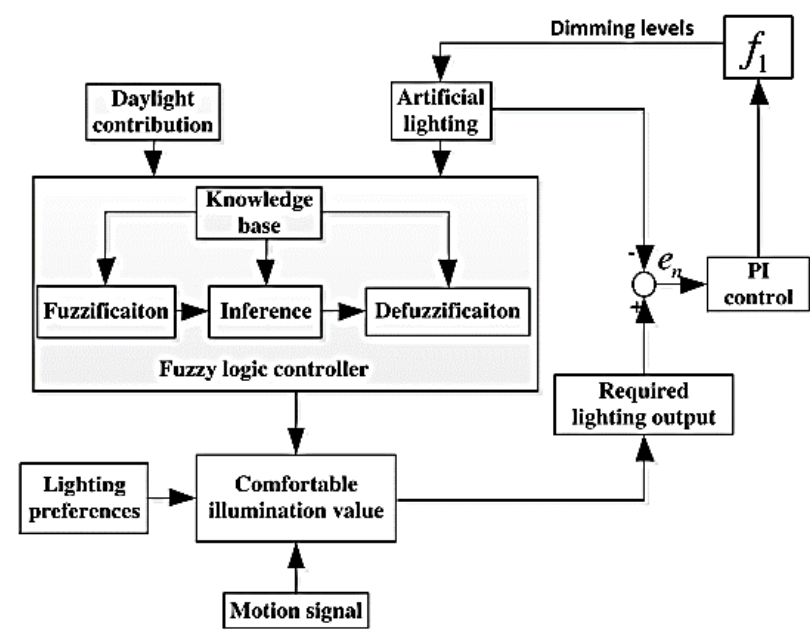

Figure 2.13 User preference consideration using fuzzy logic and utility function for lighting control [49]

The previous discussion about the several researches revolved about the controllers that different authors used to control the illumination of luminaire, or the horizontal illumination. To control the vertical illumination, the controlling concept is almost the same. Lau et al. [50] 
developed the following control system for controlling the angle of roller blind. The system was aimed to assure the illuminance of the indoor environment to be constant with error of $+/-20$ lux. This allowed the utilization of external light source to reduce the load of artificial lighting. Fuzzy logic control that took set point illumination and the error of illumination as inputs is an example of reflecting human decision on controlling the roller blind angle.

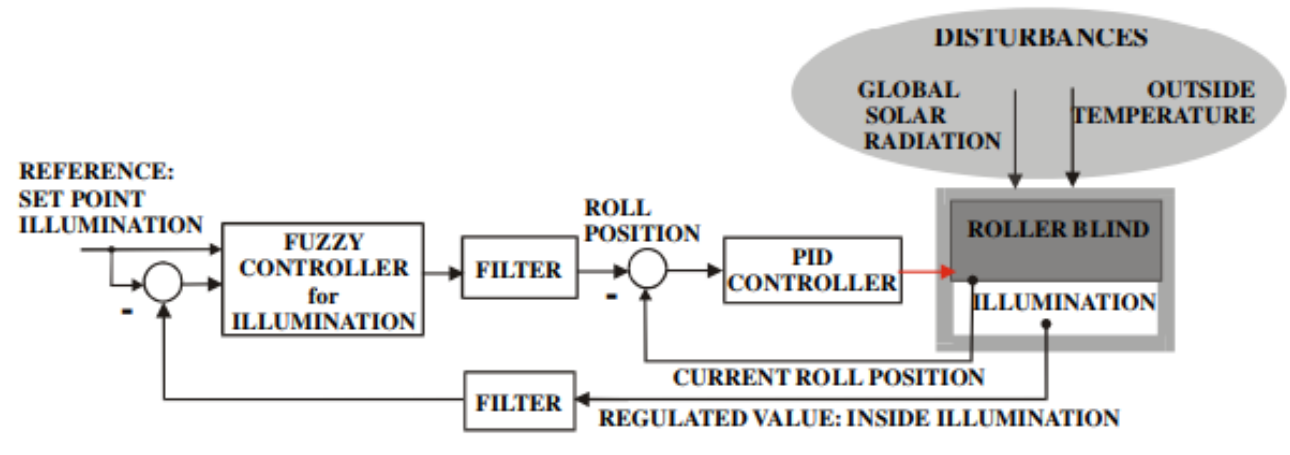

Figure 2.14 Combination of fuzzy control and PID control for vertical illumination adjustment [50]

\subsection{Summary of Literature Review}

This chapter reviewed the major work that were conducted by various scholars to address the issues related to illumination and visual discomfort in terms of glare. The fact that horizontal illumination, which is the workspace illumination, is not the only consideration for maximizing visual comfort is because various factors including color, color temperature, and glare are all major issues that can affect a person's physical and physiological comfort, simply saying "it is dark" or "it is bright" is not enough to provide occupants with comfort.

From all the attempts of visual comfort work, there has not been a single method or standard that the field has recognized as the absolute correct way due to the complexity of the perception of light to human. Although glare indices provide general scale of comfort, each index comes with its own weakness and DGIN seemed to be the best index for visual comfort 
evaluation due to its simplicity in measurement, and the daylight factor that was taken into greater consideration compared to its previous version of DGI.

In view of the complexity of visual assessment and the somehow not reliable method of visual comfort evaluation using glare indices, past research demonstrated user preferences as one of the factors for controlling the environment illumination level such as using utility function or by frequent and direct user feedback, but both methods were impractical in reality. Since this subject feeling cannot be ignored, a better way of personalizing lighting control system is necessary.

There has not been much work done to evaluate visual comfort for aircraft cabin setting. The only one that was directly related was investigating the influence of color to passengers, it dealt more with physiological response rather than investigating the eye comfort as the major topic. Furthermore, starting from the work of Kruithof, it has been recognized that color temperature is correlated with illumination that affect the general perception of brightness, for the same illuminance level but with different color temperatures, occupants would report different brightness feelings, this means that color temperature of artificial lighting needs to be integrated to set up a "comfortable environment" in a mock up cabin.

On the other hand, it was found that a lot of researchers have focused on reporting how they controlled the horizontal illumination, but for vertical illumination, only roller blind control method has been fully discussed about. There is apparent advantage of smart windows over traditional blinds, especially for electrochromic windows, due to its high contrast ratio and very low transparency when it is at its full darkness and proven effectiveness of glare elimination. It is necessary to investigate a feasible and effective method for controlling the device. Fuzzy logic 
seems to be a direct way for the incorporation of various factors that are difficult to express in mathematical terms but easy for human decisions. 


\section{Chapter 3 System Setup}

The purpose of this chapter is to propose a method for glare detection inside an aircraft cabin with the window being the only glare source. The area that is occupied by each passenger is very small and limited so that the passenger is more sensitive to glare. In this chapter, the research discussion starts with describing the setup of test environment and the placement of equipment.

\subsection{Mockup Environment}

A section of cabin environment was built with the dimensions similar to that of the Bombardier Aerospace Global 7000 series business aircraft based on a provided CAD drawing (Figure 3.1). Due to limited assembly equipment available, a simplified version that resembled the characteristics of the actual cabin was built using extruded aluminum tubing as frames. Since the research is for a business aircraft, the seating arrangements were different throughout the cabin. For this thesis, one seating arrangement was chosen (Figure 3.2).

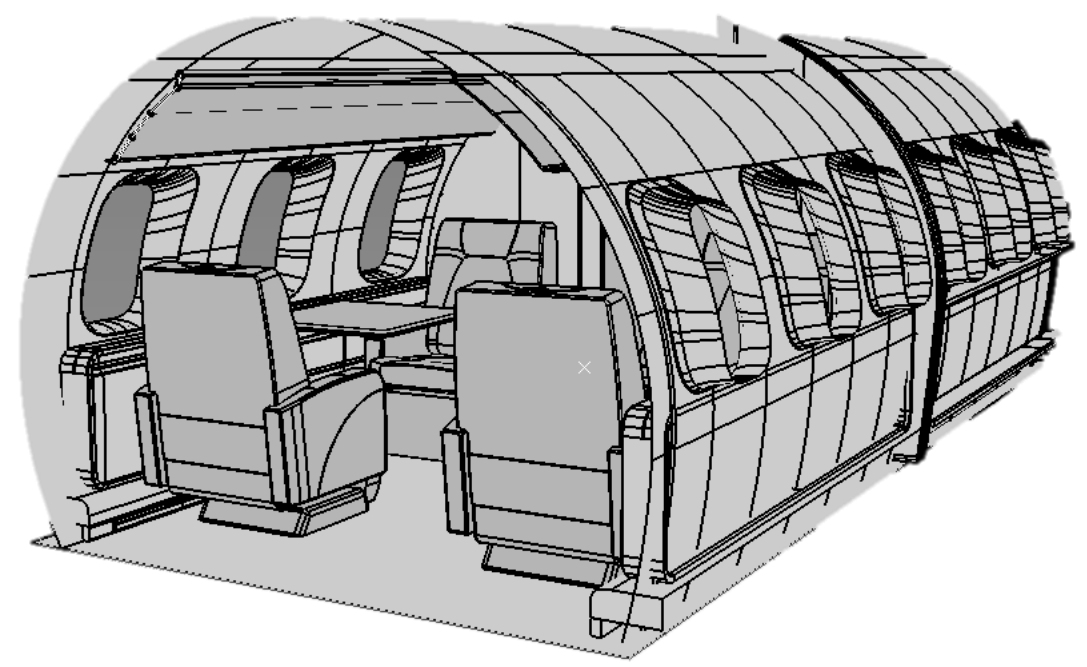

Figure 3.1 Bombardier Aerospace Global 7000 cabin environment (provided by Bombardier Aerospace) 


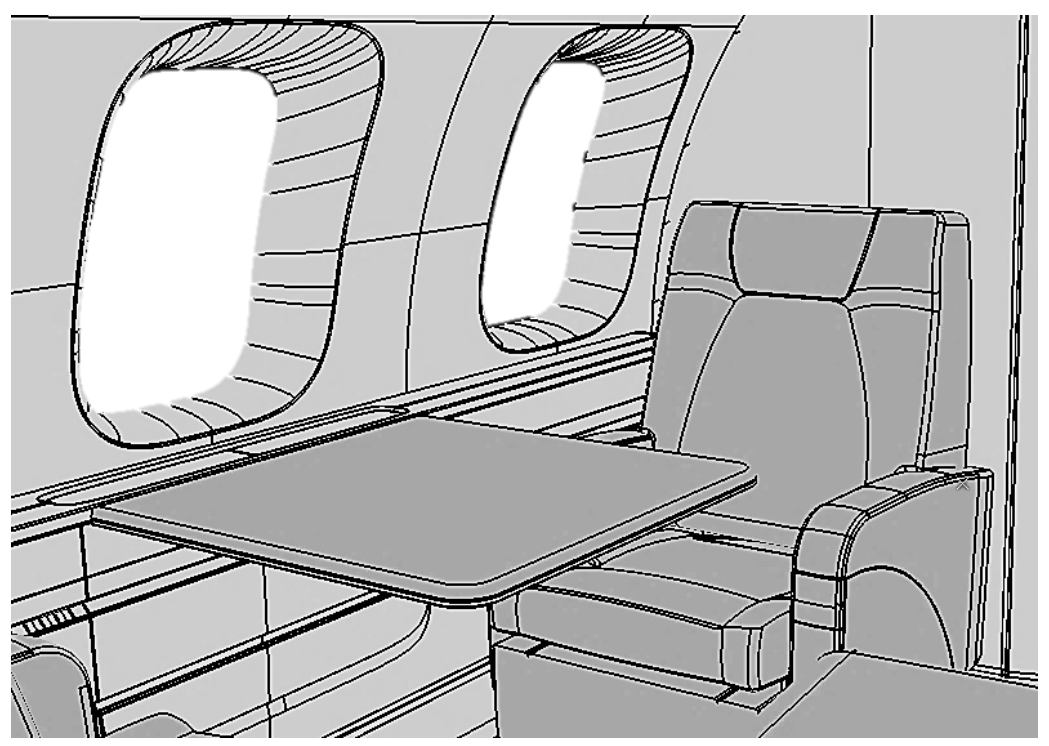

Figure 3.2 Bombardier Aerospace Global 7000 seating arrangement

The reason for this arrangement over others was because of its specific setup as well as the limitation of constructing the mock up. Firstly, the seat was orientated in a way that the passenger was facing parallel to the surface of the window to represent the actual aircraft seating. Secondly, the seat was positioned right beside the window. In this case the passenger would be most prone to the influence of daylight. This set up also created a better scenario to study the influence of both daylight and interior artificial lighting. Thirdly, there was a table present in front of the passenger which could practically be interpreted as the workplane for the horizontal illuminance detection to take place. However, the limitation was only one electrochromic window available for use. In this case, the primary concern is to reduce discomfort glare from external lighting.

The dimension of the table was $66 \mathrm{~cm}(\mathrm{~L}) \times 67 \mathrm{~cm}(\mathrm{~W})$, and it was located approximately $52 \mathrm{~cm}$ away from the backrest of the chair (Figure 3.3). Considering passenger activities could extend outside the table, for example, when the passenger was simply sitting back reading a book, 
then the actual workplane extends for another $30 \mathrm{~cm}$. Therefore, the area to be considered as a passenger personal zone was $96 \mathrm{~cm}(\mathrm{~L}) \times 67 \mathrm{~cm}(\mathrm{~W})$.

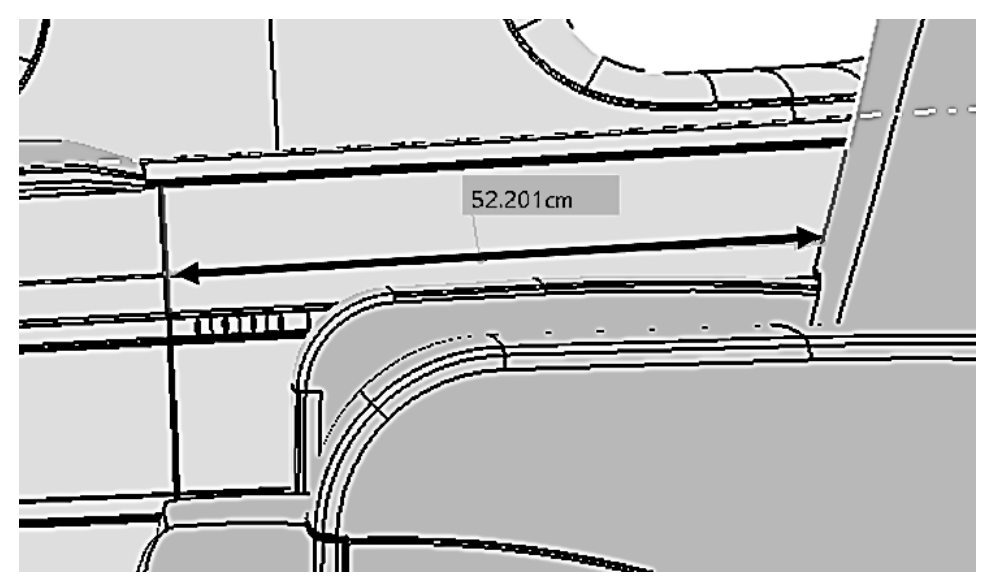

Figure 3.3 Passenger personal zone

Based on the information above, a mockup was constructed (Figure 3.4). A number of major considerations were taken during its development with the main focus on the area of illumination.

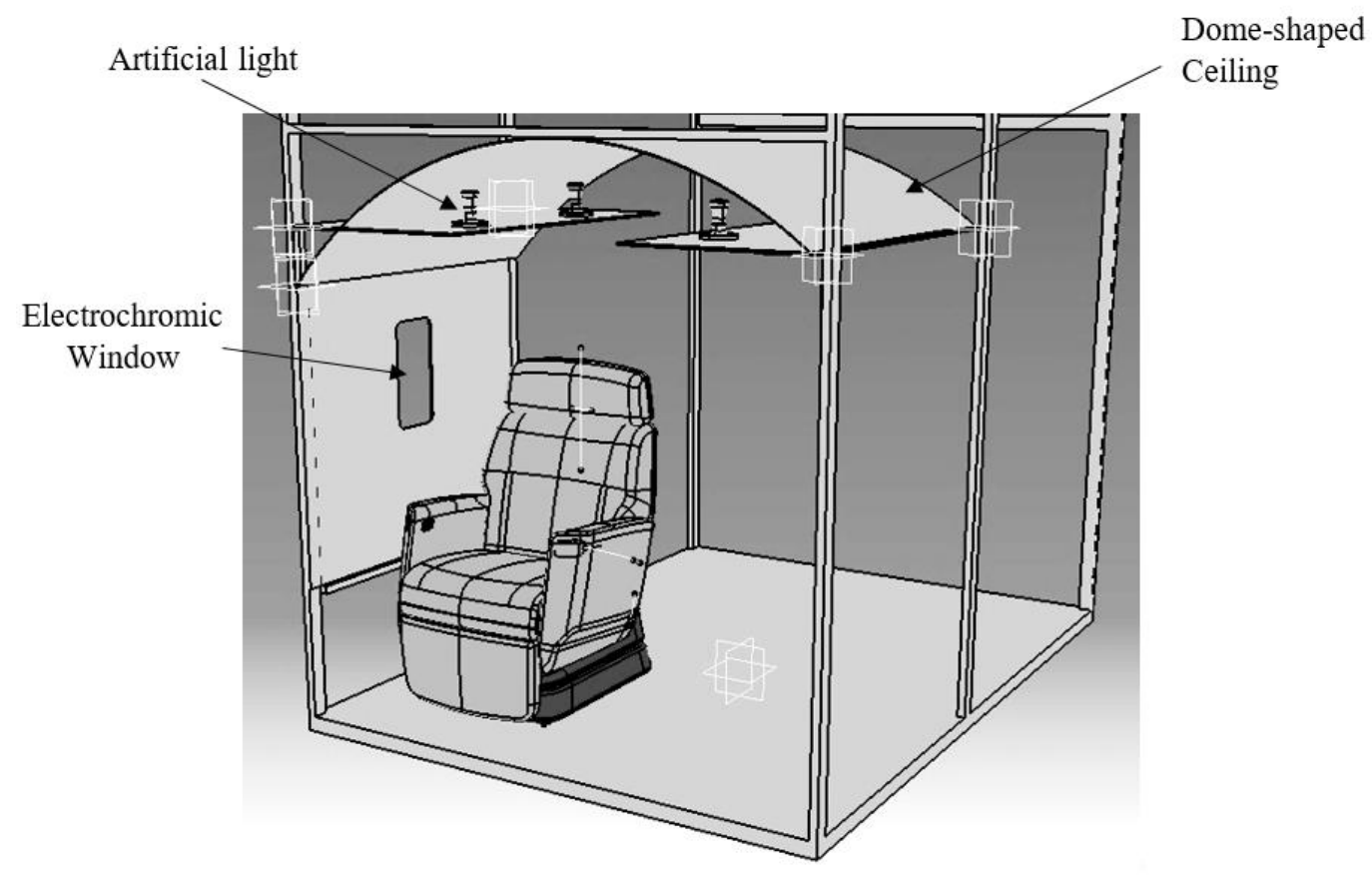

Figure 3.4 Cabin mockup environment 
The mockup was set up in a laboratory area, with a dome shaped white ceiling that resembles an aircraft cabin ceiling with a radius of 48inches. The curved ceiling could act as a concave reflective surface to retaining ambient or external light inside the cabin.

The lab environment was illuminated solely by fluorescent lamps without any sun light contribution, with an average horizontal illuminance at working height, $75 \mathrm{~cm}$ above ground. An average of 420 lux was detected using a photodiode (the preparation of the use of diode will be discussed later in section 3.4). There was no control of variation in illumination except for turning on and off the lights. That could hardly be used as a light source to simulate various glare scenarios for testing. What was needed was an enclosed area that could minimize the contribution of the uncontrollable lighting and allow the light sources to be dedicated for the experiment within the set up.

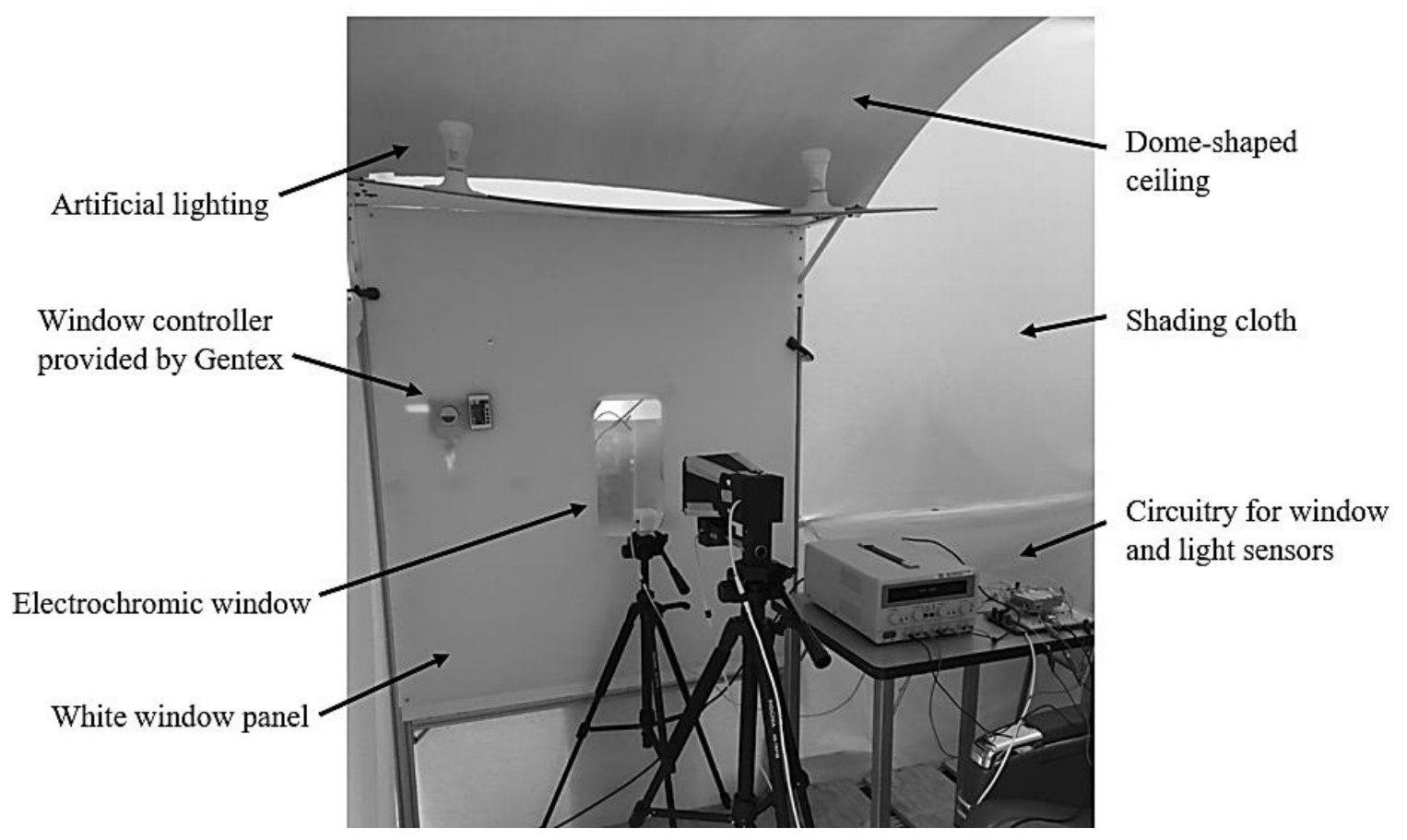

Figure 3.5 View from inside the mock up 
In order to do so, the cabin mockup was covered entirely with light brownish-yellow cloth (Figure 3.6). Not only was the cabin environment isolated from the surroundings, but also the overall color temperature was warmer which allowed the occupant to easily feel more comfortable when the window dim level was adjusted. The occupant sense of discomfort would not be affected by the cabin color itself. Otherwise no matter how the window dim level was adjusted, the occupant might claim to be uncomfortable. Furthermore, white cloth was not chosen because it could cause the color temperature in the set up to vary by few thousand Kelvins [40].

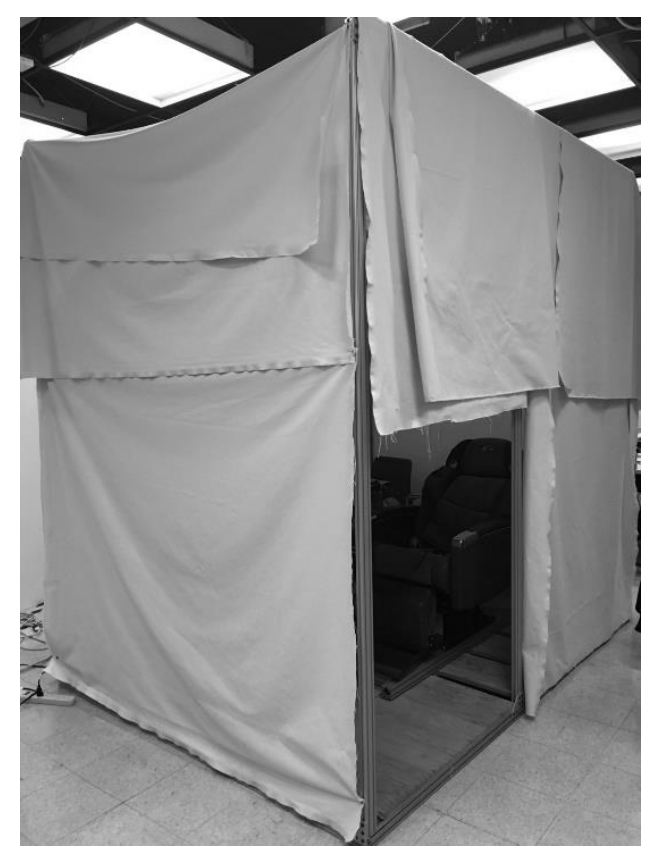

Figure 3.6 View from outside the mockup

According to a window design by Gentex (Figure 3.7), the placement of the EC window should be between the dust panel and the aircraft structural window. It was assumed that the light transmissibility percentage was close to 1 . Therefore, during the mockup design, except for the electrochromic window itself, the influence of other window components on light transmissibility was considered negligible. Therefore, they were not installed in the mockup. 


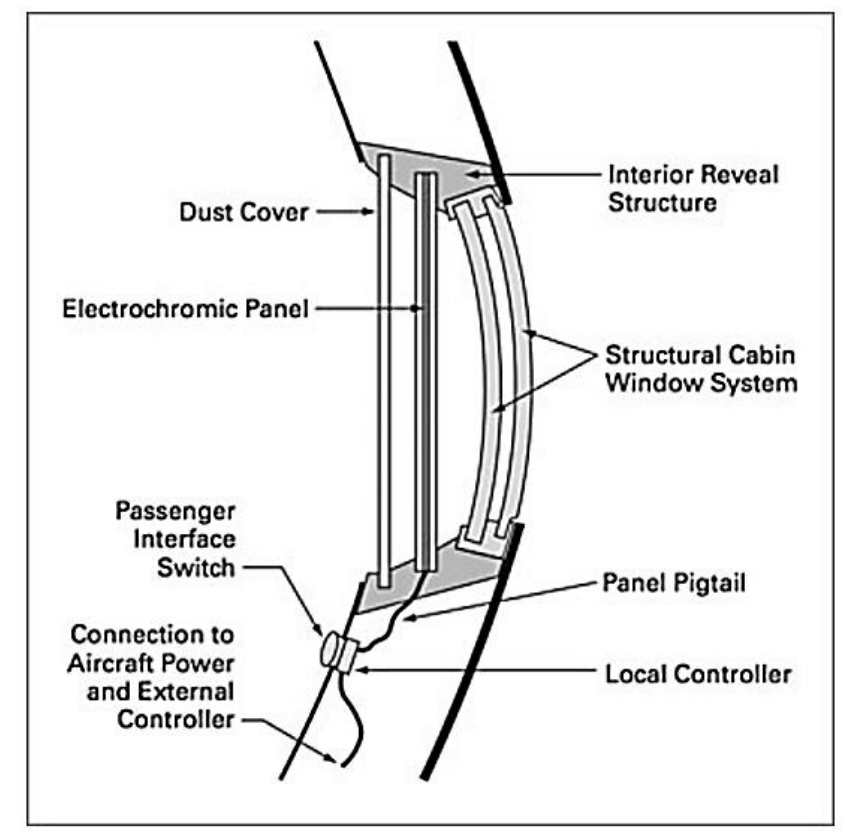

Figure 3.7 Placement of electrochromic window in aircraft cabin (picture provided by Gentex)

\subsection{Testing the Properties of Electrochromic Window}

The EC window sample used for this project was manufactured by Gentex. This window has a dimension of $28.5 \times 47.5 \mathrm{~cm}$ in an oval shape, and the actual usable dimension is $36 \times 18$ $\mathrm{cm}$. The only information provided about this window is $99.99 \%$ blockage of the visible light when $1.2 \mathrm{~V}$ is applied to dim it to the darkest. However, due to the high light intensity that would be encountered at high altitudes, this could still mean a significant level of visible light that would be noticed by occupants. It was expected that such level can be as high as 100 lux. In this research, the experiment was conducted indoor and there was no available artificial light source that could emit light intensity as high as 10,000 lux, which is the lowest usual daylight illuminance level. The principle of this project is to provide a methodology to maximize passenger comfort. The same procedures could be applied to different EC windows by adjusting appropriate parameters. An assumption was made that the artificial external light source would be completely blocked by the electrochromic window. This was true since the maximum 
illuminance that could be detected from the light source was only 3000 lux that the EC window could shield.

EC window operates based on chemical reaction from $0 \mathrm{~V}-1.2 \mathrm{~V}$ with $0 \mathrm{~V}$ being the clearest and $1.2 \mathrm{~V}$ being the darkest and the window remains fully clear under $0.3 \mathrm{~V}$. In order to change from clear to dark, a voltage that corresponds to the respective tint has to be applied directly to the window and the chemical reaction inside would take place. However, from understanding the window experimentally, in order to change the window from dark to clear, a polarity inversion is required to trigger the reverse of reaction. Then the positive voltage of the respective desired tint state is applied and the window would start bleaching. If this step is neglected, the initial transition time would be much longer.

To illustrate the window performance, a test was done by placing a sensor right below the window (Figure 3.8) and allowed the window to undergo the transition from fully tinted to fully clear state and vice versa. It took approximately 220s (Figure 3.9) for the whole process to complete. During the first 30 seconds, less than 25 lux change was detected. This change would normally be too small to be noticed. To the author's knowledge, there is no other effective way to speed up the process. On the other hand, it took less than 90 s to transition from clear to fully tinted (Figure 3.10).

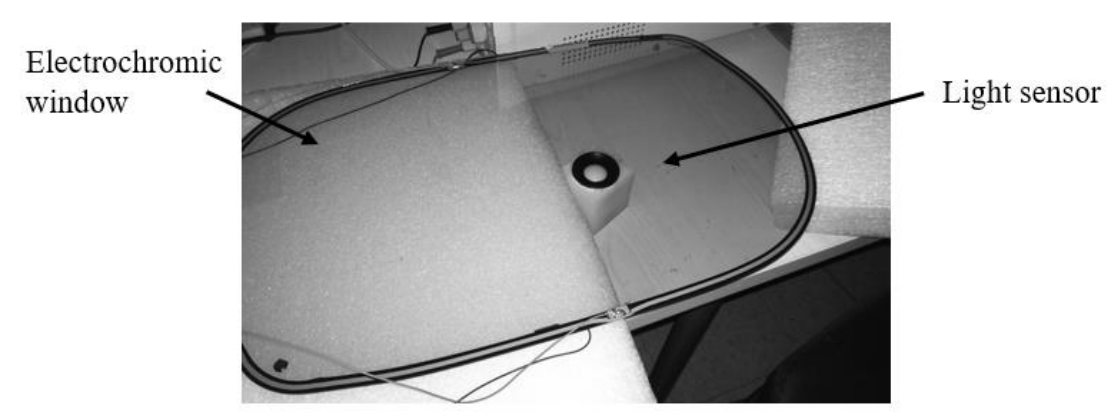

Figure 3.8 Window dimming and clearing test 


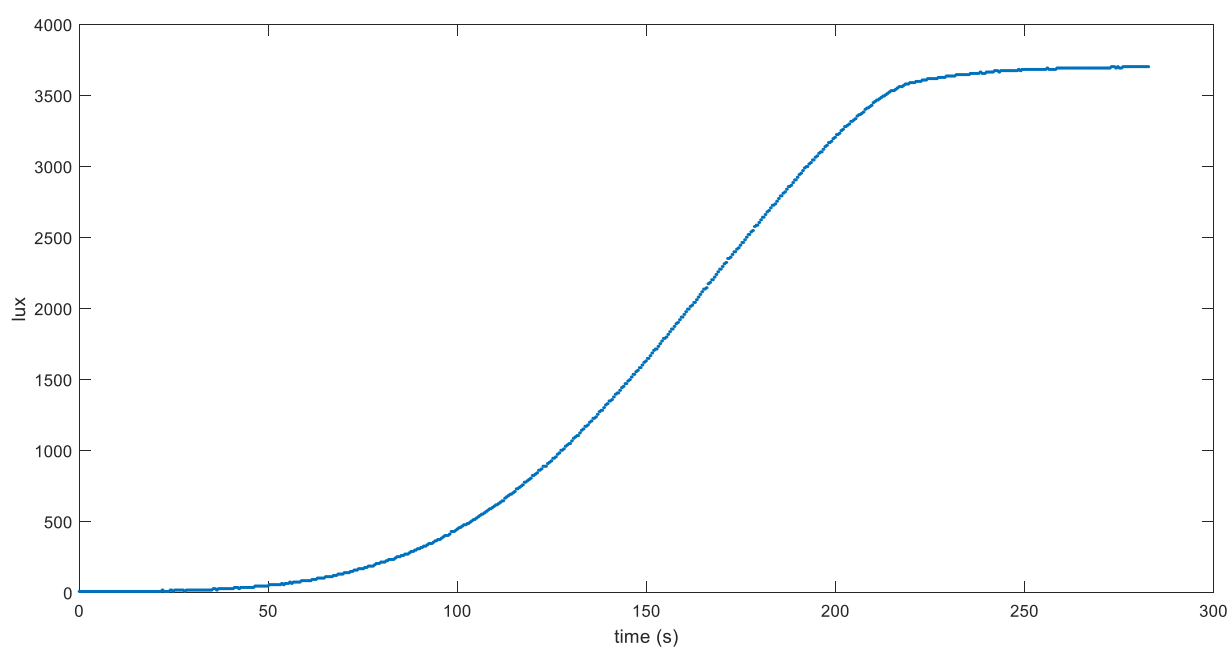

Figure 3.9 Electrochromic window from fully dimmed to clear

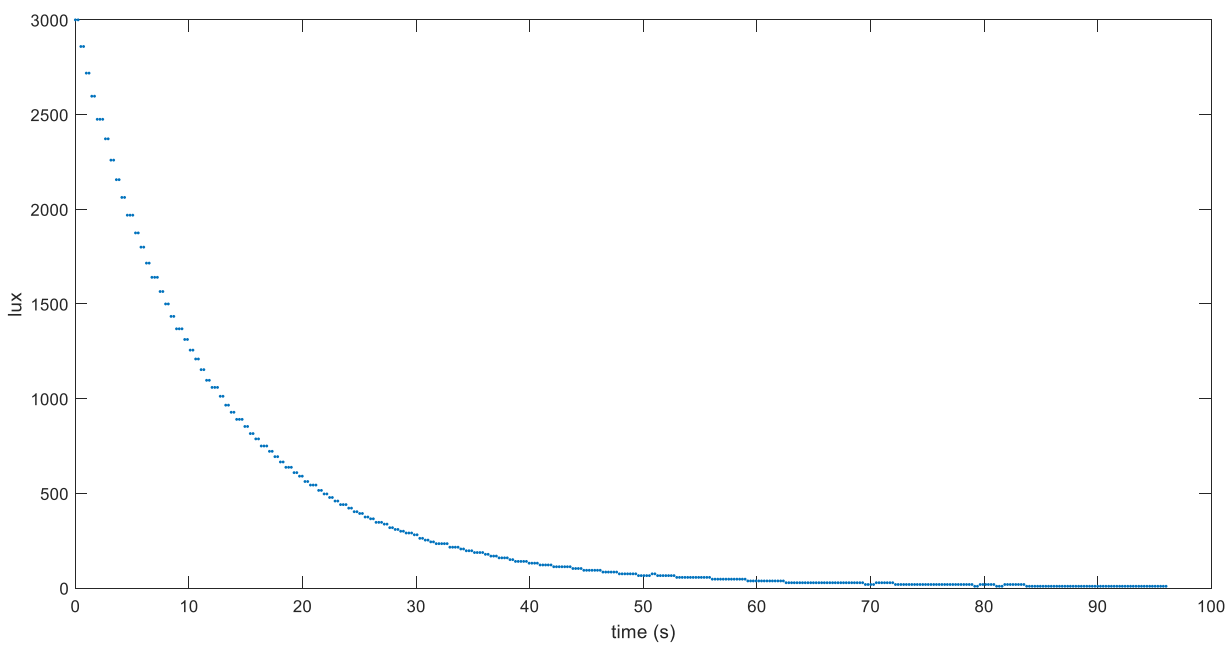

Figure 3.10 Electrochromic window from clear to fully dimmed

The power supply for the EC window was a controller provided by Gentex (Figure 3.11). It consisted of four buttons that allowed the user to manually select four levels of dimness. It did not contain other functionalities that allowed users to choose between intermediate dim levels. In terms of continuous tint control, it was determined that a RoboteQ (RQ) motor controller be an appropriate device for the task. 


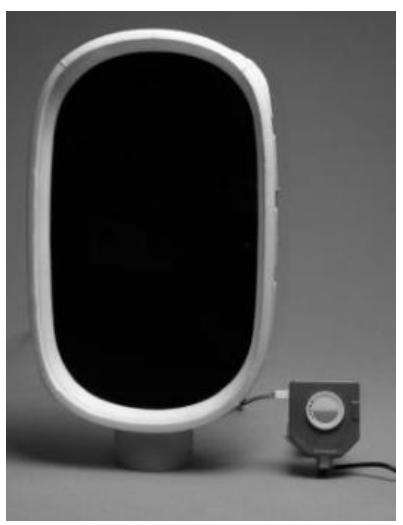

Figure 3.11 Electrochromic window manual controller provided by Gentex

The RQ controller is capable of outputting voltage that is linearly proportional to the desired percentage in a resolution of $0.1 \%$ (Figure 3.13). The lowest voltage that the RQ controller receives is $8 \mathrm{~V}$, and the controller outputs the percentage of voltage that corresponds to the input. Since the operating limit of the window is $1.2 \mathrm{~V}$, the range of usable values of the RQ controller percentage is from $0 \%$ to $15 \%$, and to command the RQ controller output, the range read by the controller is 0 to 150 .

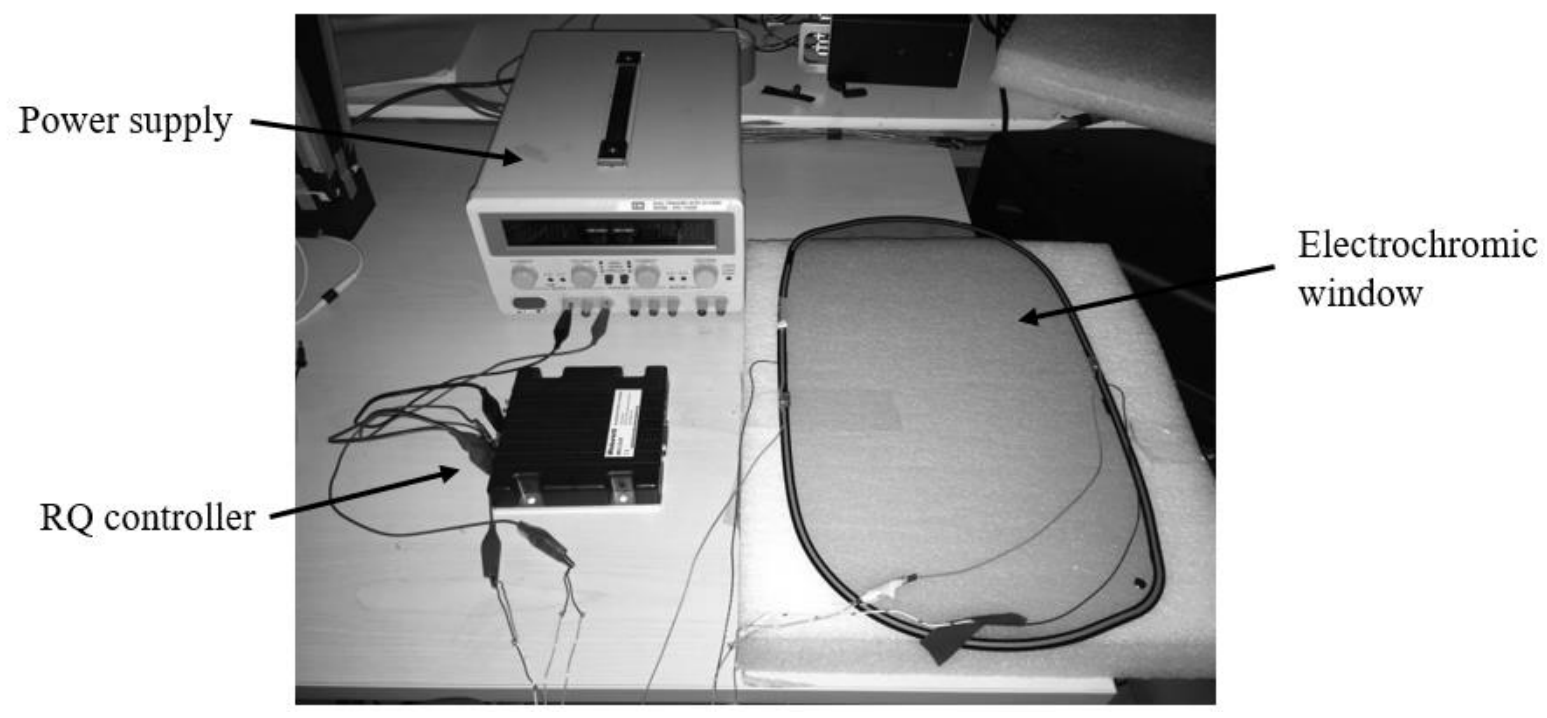

Figure 3.12 Picture of RoboteQ motor controller connecting to the electrochromic window 


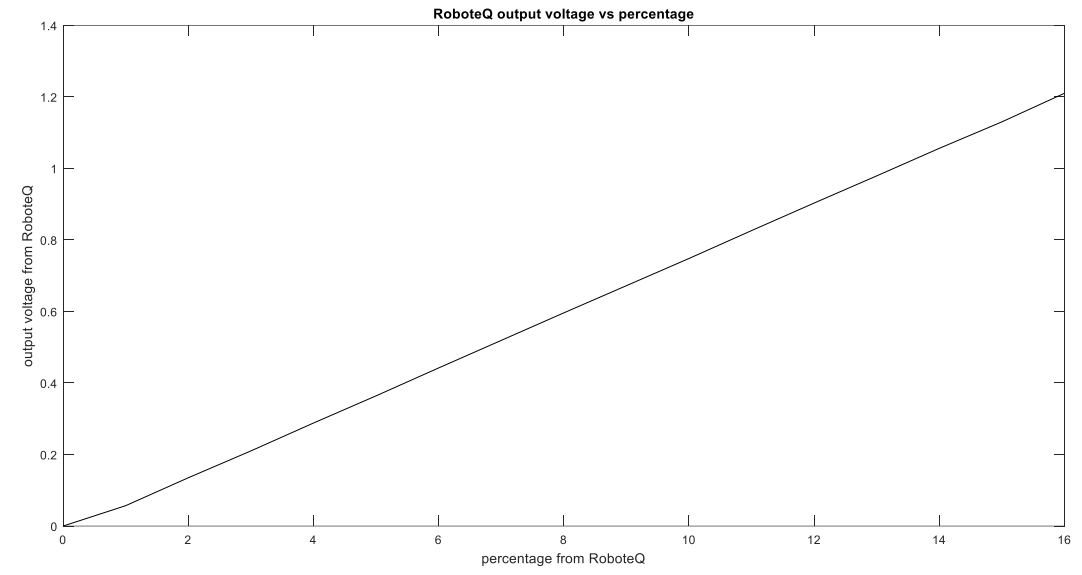

Figure 3.13 RoboteQ motor controller output voltage vs percentage of controller input voltage

A problem was noticed when using the RQ controller to control the window. When the RQ controller continuously powered the window, the window eventually turned to its dimmest when it was not supposed to. This happened when a sufficiently high voltage was applied, approximately at $0.7 \mathrm{~V}$, which caused set point settling problems. In regards to this, the developed software that controlled the RQ controller included a stop time between each feed to prevent this situation from happening. The stop time was carefully chosen by performing a number of tests. A stop time that was too long would cause the RQ controller not able to maintain a stable tint since the window had a tendency to return clear without voltage input. When a stop time that was too short, it would not be able to solve the mentioned problem. The stop time finally chosen was $0.2 \mathrm{~s}$.

\subsection{Lighting Setup}

Figure 3.14 shows the back of the mockup where an external light source chamber was located. It was constructed using white foam boxes for the high reflectance surfaces. A chamber was required such that the external lighting as seen by the user from inside the mockup was consistent in terms of the color temperature perceived as well as in terms of the overall 
luminance. The chamber blocked the view of the laboratory environment such that the occupant would not get distracted by other objects.

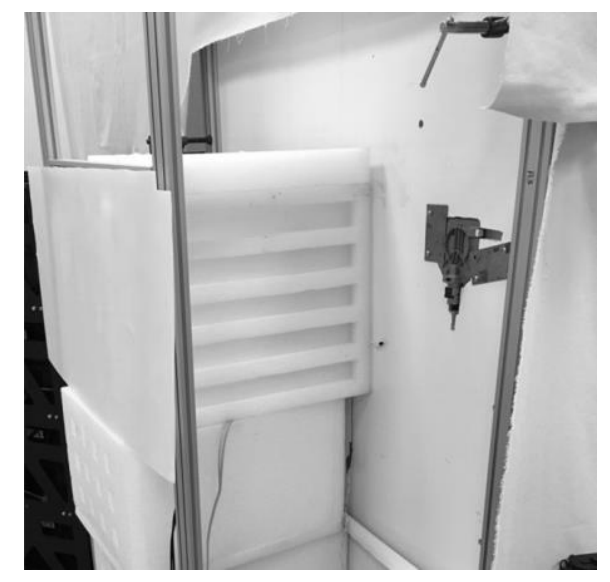

Figure 3.14 External light source chamber

Inside the chamber (Figure 3.15) two Philips Hue LED bulbs were installed with each emitting 840 lumens. The benefit of this light bulb was that color, color temperature and light intensity were wirelessly controllable which favors the re-making of various scenarios for glare testing. Aluminum foil layers were inserted surrounding the window to further increase the glare effect.

With the setup using the above description, it was noticed that from the inside of the mockup, the external light source consisted of two separate concentrated spots rather than a uniform and evenly distributed lighting area. This would cause the occupants to feel higher discomfort than the values reflected. On the other hand, the light sensors assume an evenly distributed light area for calculating glare. Therefore, it was required to make adjustment in order to remove the concentrated effect. This was solved by adding a condenser lens between the light source and the window, and also adding a concave mirror behind the light source (Figure 3.16). This concept of optics enhancement was taken from the design of overhead slide projectors, in 
which the glass surface where the film slides was evenly illuminated because of such lighting setup. The contrast of before and after having the optics enhancement is shown in Figure 3.17.

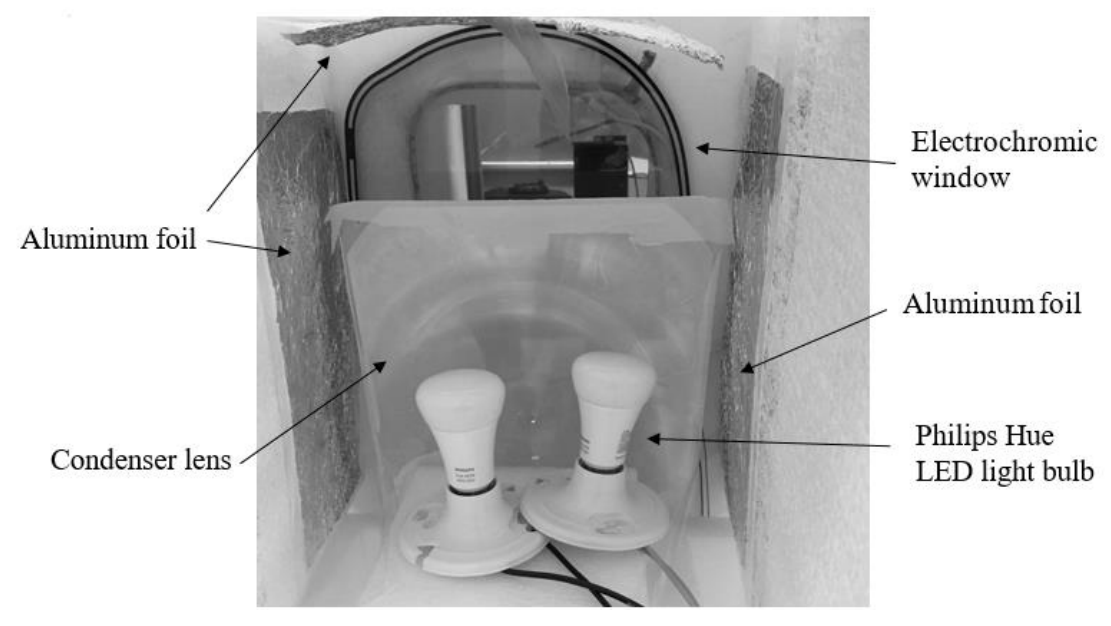

Figure 3.15 Inside the external light source chamber

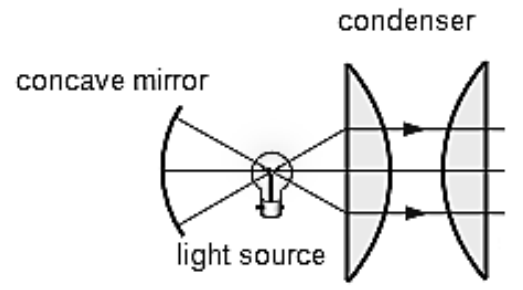

Figure 3.16 Illustration of optics enhancement for the light chamber
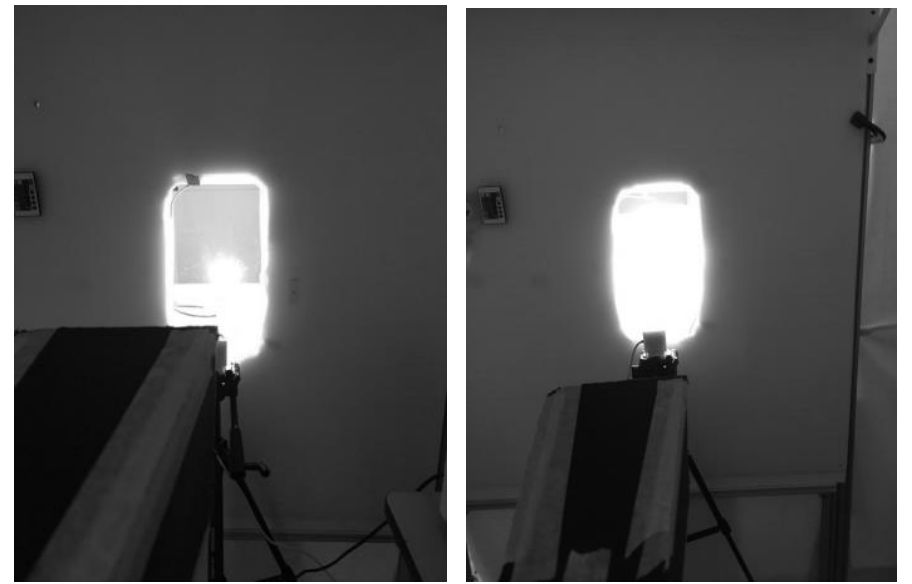

Figure 3.17 View from inside the mock up without optics enhancement (left) vs with optics enhancement (right) 


\subsection{Light Sensing}

The glare detection method chosen for this project was DGI method proposed by Nazzal for the reason mentioned in Chapter 2. The following is a summary of the experiment setup. The glare testing unit consisted of three light sensors and an electrochromic window. The sensors used were spot sensors to detect light mainly coming in from one specific direction. Each sensor was responsible for sensing different light component. The first sensor was to detect the vertical luminance of the window $\mathrm{L}_{\text {window }}\left(\mathrm{cd} / \mathrm{m}^{2}\right)$. Since this sensor should not detect any lighting from other sources, it was shielded with a pyramid made of a black and irreflective material. The construction of the black box will be described shortly. The second sensor was to detect the average vertical luminance of the surroundings or the adaptation luminance $\mathrm{L}_{\text {adaptation }}\left(\mathrm{cd} / \mathrm{m}^{2}\right)$, and the third sensor was to detect the vertical luminance $\mathrm{L}_{\text {exterior }}\left(\mathrm{cd} / \mathrm{m}^{2}\right)$ caused by diffused sunlight and any reflection from outside.

Before setting up the equipment, the shield (circled in Figure 3.18) for sensor 3 needed to be built. Since it was made of a black and non-reflective material, a black cardboard with the needed rigidity for a box in a pyramid shape was chosen. In addition, an additional layer of black cloth was placed on the surface to eliminate the reflectance.

The angle of the opening of the pyramid should be directed to the edges of the EC window, and placed at the distance $d$ between the window and the observation place [51]. This distance was set at $67 \mathrm{~cm}$, i.e. the width of the table mentioned in section 3.1. According to the geometry calculation demonstrated by Nazzal, the smallest possible bottom opening of the pyramid was $5.9 \mathrm{~cm}$, the pyramid depth $d^{\prime}$ was $20.6 \mathrm{~cm}$, and the wider opening $a^{\prime}$ of the pyramid was $9.4 \mathrm{~cm}$. 


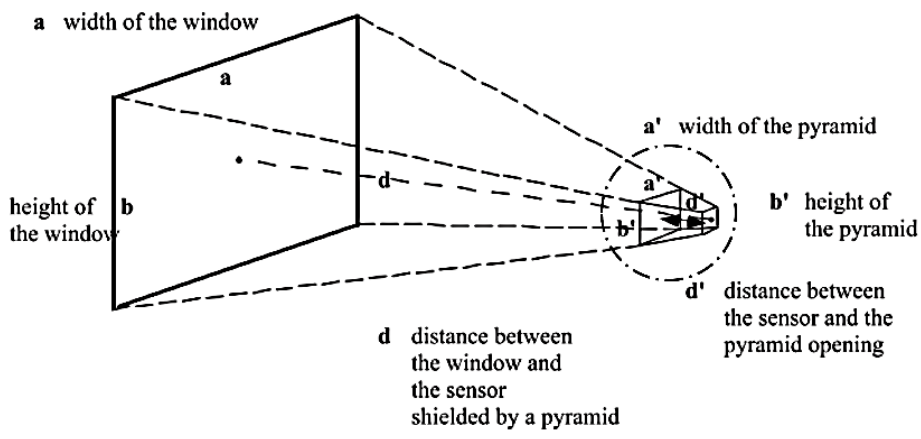

Figure 3.18 Shape of pyramid (black box)

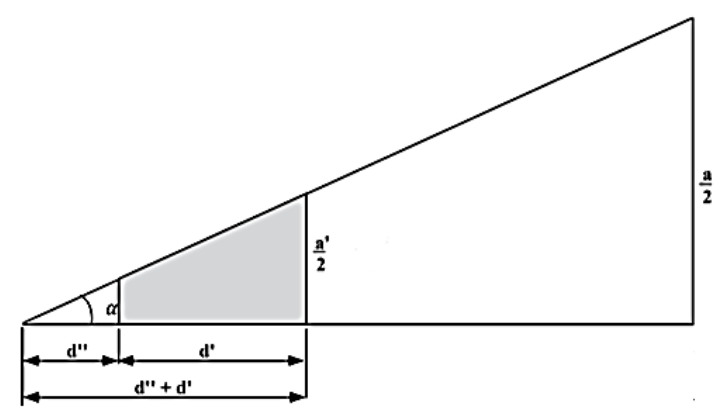

Figure 3.19 Dimensions of pyramid

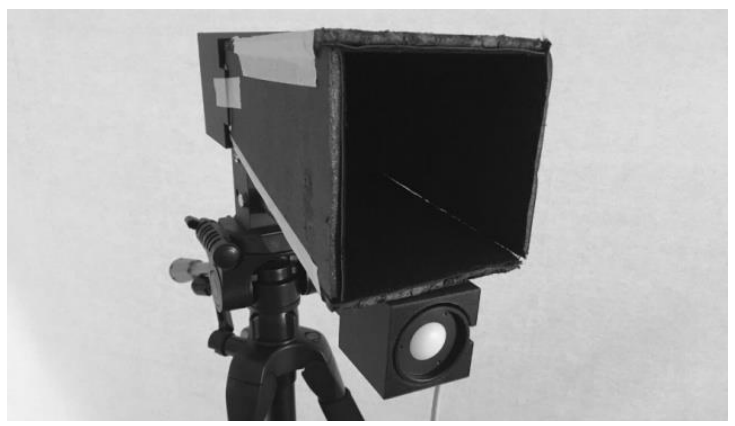

Figure 3.20 Picture of the light sensing pyramid

The following describes the placement of the sensors. Sensor 1 was placed right in front of the window located right below sensor 2's line of sight. Sensor 3 was placed at the back of the pyramid from the specified location mentioned above. Sensor 2 was placed right under the opening of the pyramid. Figure 3.21 shows the schematic of the set-up, and Figure 3.22 shows the actual setup that was done. 


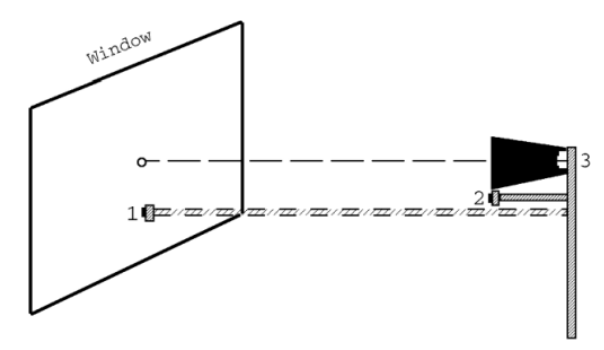

Figure 3.21 Placement of the three sensors and the pyramid [7]

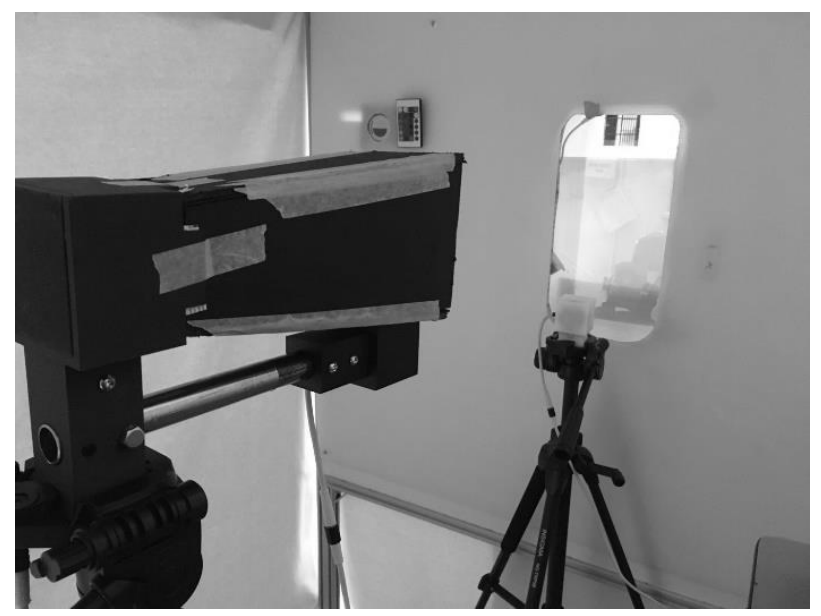

Figure 3.22 Picture of DGI detection setup

The three spot sensors used for the experiment were the same product from Solar Light Company. They were analog photonic sensors that could detect light from 0 to 150,000 lux and the effective wavelength range was $380-780 \mathrm{~nm}$ with photonic response similar to human eyes (Figure 3.23). The company performed calibration at $2 \mathrm{klux}$, and the sensor would output $4 \mathrm{~mA}$ when the illumination is at 0 lux and $20 \mathrm{~mA}$ at 150 klux. The advantage of this sensor is that it was able to detect a wide range of light intensity linearly proportional the current output. Signal was collected using a $4-20 \mathrm{~mA}$ data acquisition board to communicate with a computer via a RS232 to USB port converter at a baud rate of 9600. 

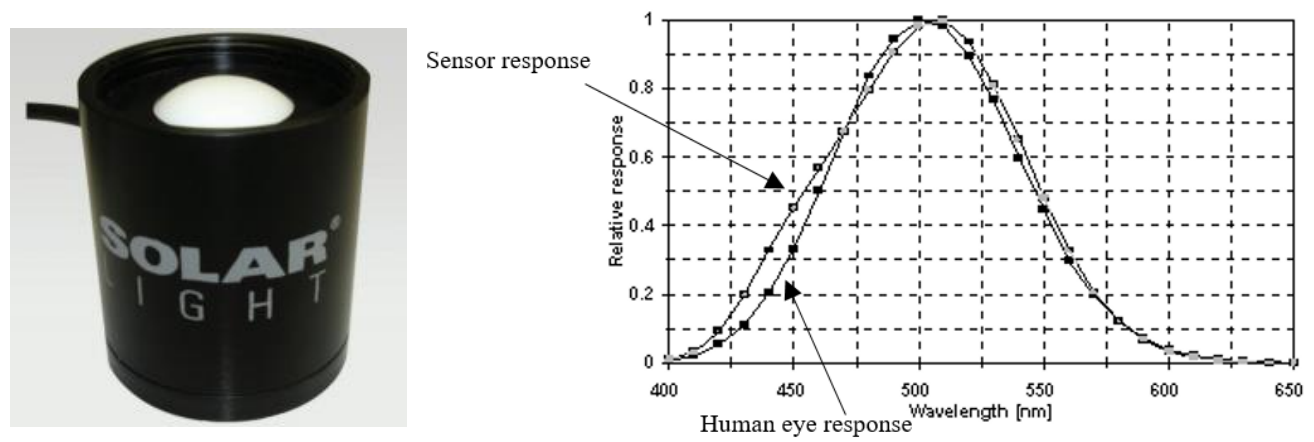

Figure 3.23 Light sensor and its response curve

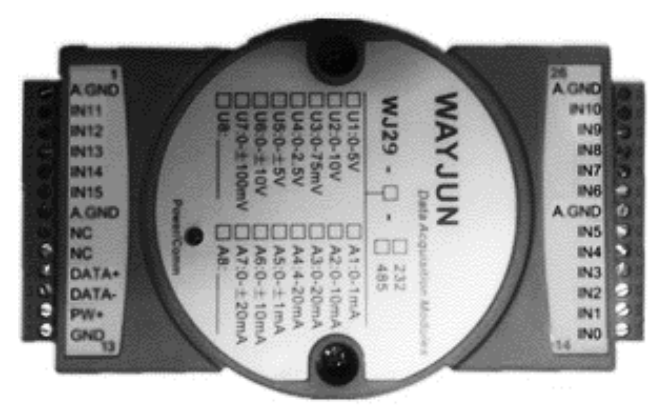

Figure 3.24 An 8 channel 4-20mA data acquisition board

To measure the horizontal illumination on a workplane as mentioned earlier, a reverse biased photodiode was used. Compared to a spot sensor used to calculate the value of DGIN, the advantage of photodiode is that it considers light from any direction that falls on to the receptor area of the photodiode sensed. The photodiode chosen for this project was a Panasonic AMS302 light sensor capable of sensing illuminance ranging $3 \mathrm{~lx}$ to $1,100 \mathrm{~lx}$ with photocurrent linearly proportional to illumination. Referring to Table 2.3, the range of horizontal illuminance is normally required for usual activity that a passenger would do on a plane such as reading and resting at less than $750 \mathrm{~lx}$. This is well below the operation limit of the diode. Another property of this diode was the dependency of the responsiveness on the wavelength that falls under the 
detection area. The response curve was similar to that of the photonic vision curve of human eye, with the visible light wavelength ranging from 350 to $800 \mathrm{~nm}$.

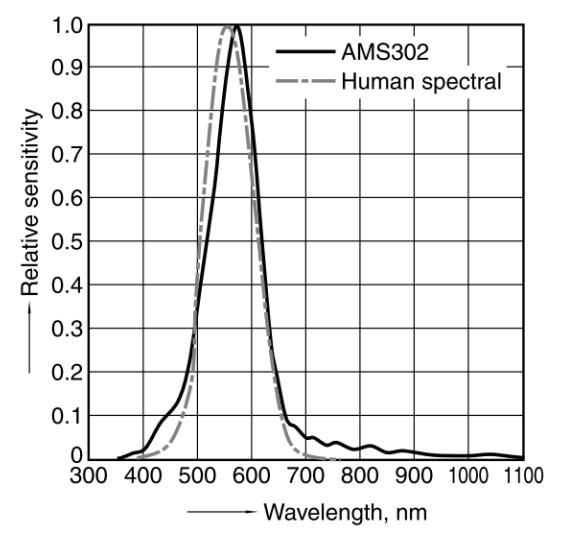

Figure 3.25 AMS302 photo diode response sensitivity vs wavelength

The circuit for the operation of this diode is shown in Figure 3.26. $V_{0}$ is the voltage across the resistor of an appropriate resistance and $I_{L}$ is the current output from the photodiode. The fact that this diode was "reverse-biased" means that the current that flows from $V_{c c}$ was blocked and theoretically $I_{L}$ was solely the photocurrent. Since photocurrent was relatively weak compared to a "forward-biased" diode, the contribution to the overall current flowing through the resistor was much higher and made it easier to detect. However, since the diode was thermal sensitive, there was a leakage of the current even in the absence of incident light. At room temperature, the dark current was approximately $0.004 \mu \mathrm{A}$. Since the useful photocurrent ranges from $8 \mu \mathrm{A}$ to $5 \mathrm{~mA}$, the dark current was small enough deemed negligible.

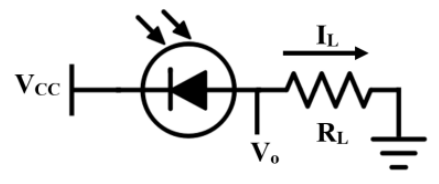

Figure 3.26 Circuit diagram for Panasonic AMS302 photodiode

The value of $R_{L}$ was not given and therefore had to be chosen experimentally. As the absolute maximum useful photocurrent was $5 \mathrm{~mA}$, the theoretical minimum value of $\mathrm{R}_{\mathrm{L}}$ to avoid 
an excessive voltage applied on the diode was $100 \Omega$. But to be safe, the test started with $110 \Omega$. After shining the light of adjustable brightness onto the diode in a dark box, the plots of the current versus the increasing illuminance were obtained with various resistors. Referring to Figure 3.27, the resistance that was too high would cause saturation at high illuminance value before the maximum photocurrent reached $5 \mathrm{~mA}$. A resistance too low would cause irresponsiveness at low illuminance value and the current would not be proportional to increasing illuminance. There would be large fluctuations in readings, leading to misreading of the illuminance values taken from the resistors of incorrect resistance.
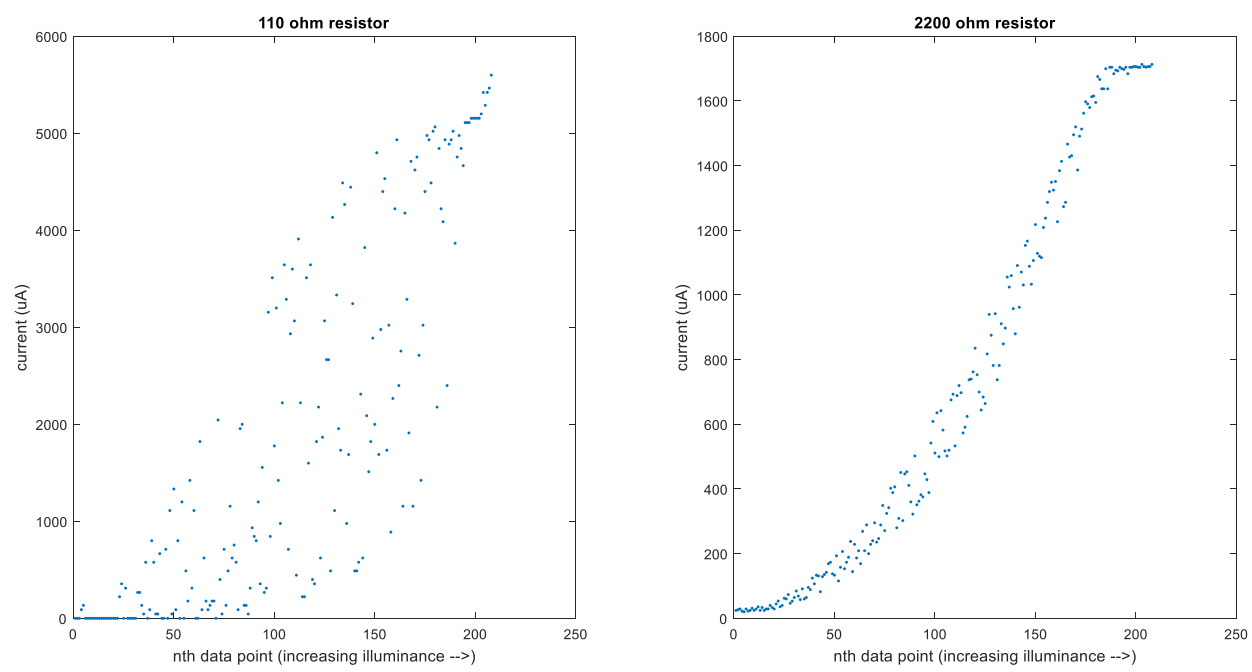

Figure 3.27 Resistance too low (left) vs resistance too high (right) for photo diode circuit

In contrast, a properly selected resistor for the circuit would solve the aforementioned problem. As seen in Figure 3.28, over $5 \mathrm{~mA}$, the higher the illuminance, the higher the photocurrent. At low illuminance environment, the detected photocurrent was not always close to zero as demonstrated by a $110 \Omega$ resistor. Under a strong luminous power, the saturation of current would not matter, because any current of magnitude higher than $5 \mathrm{~mA}$ was not useful according to the data sheet. Also, the current of 5mA corresponded to roughly $1,8001 \mathrm{ux}$. It was 
not expected that under proper control of lighting, the horizontal illuminance of workplace would exceed 600lux. Therefore, $520 \Omega$ resistor was a highly possible choice for this specific photodiode with a Vec of $5 \mathrm{~V}$. Together with a log-log plot of photocurrent versus approximate illuminance (Figure 3.29), which demonstrated a highly linear relationship with $\mathrm{R}^{2}=0.9911$, it was concluded that $520 \Omega$ of resistance was the appropriate choice for the circuit.

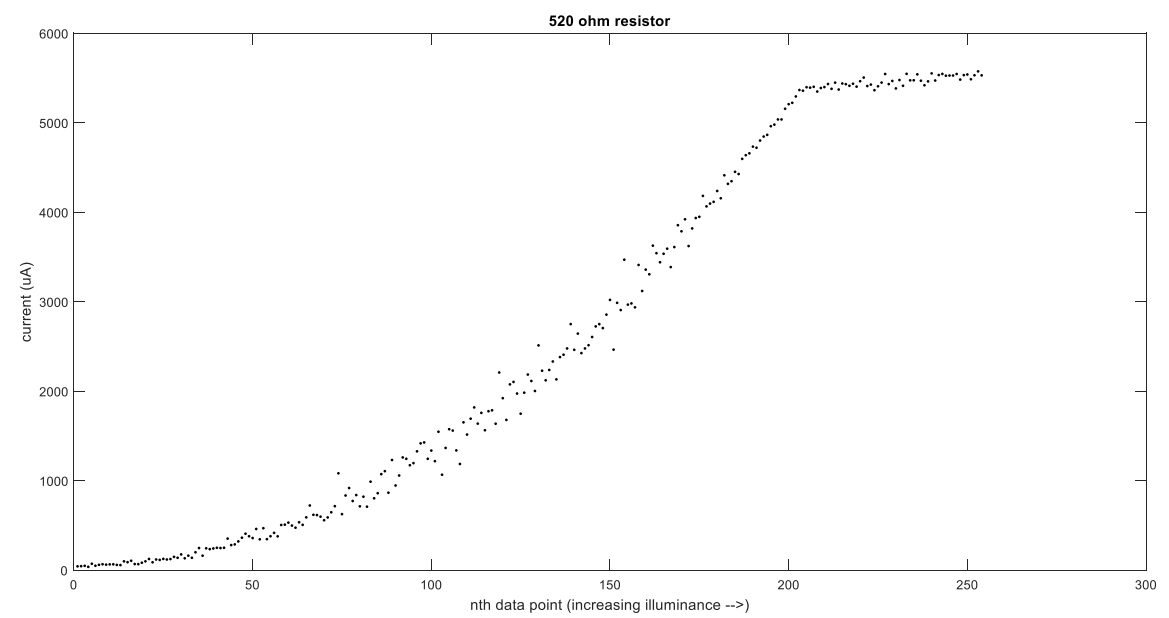

Figure 3.28 A proper choice of resistor for photo diode circuit $-520 \Omega$

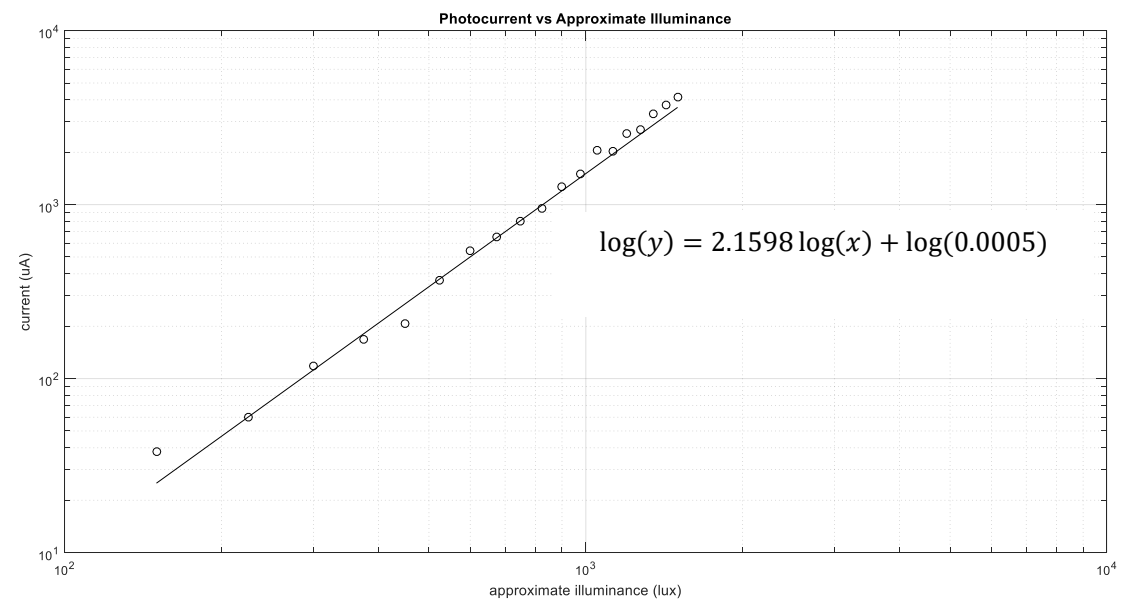

Figure 3.29 Photocurrent versus horizontal illuminance 


\section{Chapter 4 Fuzzy Logic Control}

\subsection{Fuzzy Logic Introduction}

As discussed in preceding chapters, the measurement used for discomfort measure is DGI, The DGI values can be used to form the membership functions in fuzzy logic and the output of the fuzzy logic can adjust the transparency of the window for control.

Fuzzy logic was chosen because of the fact that it mimics human decision making. Human often comment uncertain things using words such as "kind of", "somehow". In the case of glare perception, similar scenario applies. Unless the glare is so high that it is definitely not comfortable, we use descriptions like "it is a bit irritating". In daily life, we use this logic to adjust the roll angle of Venetian blinds (Figure 4.1), and the same logic behind adjusting the transparency of a smart window can be used.

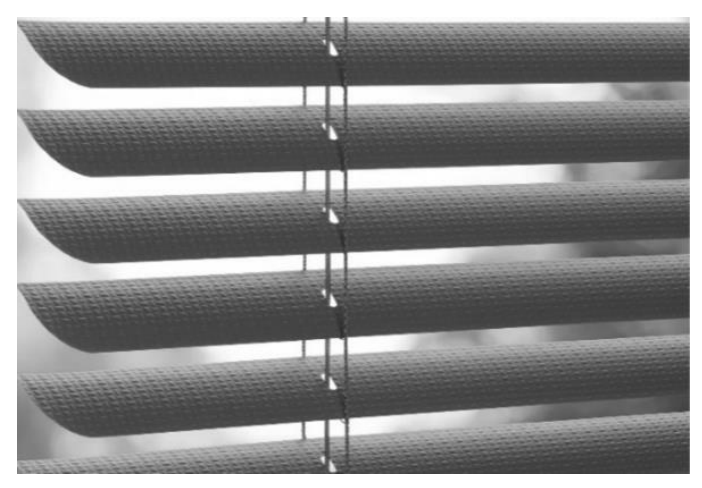

Figure 4.1 Picture of Venetian blinds [52]

The logic behind controlling a Venetian blind is based on how uncomfortable the occupant feels. Rolling the blinds downwards lets in more sunlight. But if the sun is too bright, then the user will roll the blind upwards until there is a point or a certain range that the user does not feel the discomfort glare anymore. Then one will stop rolling and keep that angle there until 
after some time the room is too dark or the current roll angle is not enough to get rid of the discomfort glare.

In terms of a smart window, the logic is the same. Instead of rolling the blinds, or dealing with the blind's angle, the parameter to be adjusted is the transparency. The window is dimmed until the user feels that the discomfort glare is eliminated and not necessary to dim it further.

\subsection{Fuzzy Logic Classification}

A fuzzy logic control method for adjusting the transparency based on various measured DGI ranges was proposed, and Figure 4.2 shows the results. The idea was that various DGI ranges which reflected different levels of discomfort corresponded to different window transparencies (Figure 4.3). But there were few problems associated with the design.

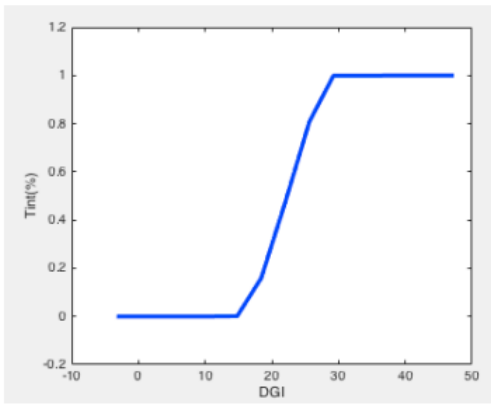

Figure 4.2 Fuzzy logic window tint classification result

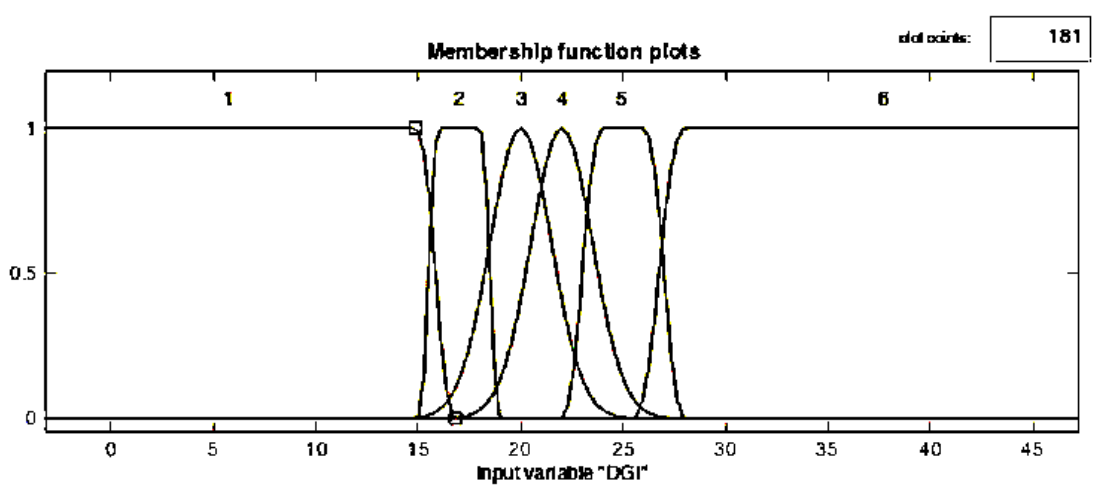

Figure 4.3 Fuzzy logic membership functions with DGI classification 
The first problem was the meaning of the curve. It implied that at a specific measured DGI, the corresponding window tint should always be at the corresponding value such that the user would not experience visual discomfort. The other way to interpret this graph is that in order to achieve a specific DGI value, the window should be tinted to the corresponding level. Either statement was based on the assumption that the luminance conditions would always be the same. Therefore, after knowing the user preference, a relationship of how the user reacted and the operation of the window could be drafted out. In reality, this would never happen as the lighting conditions are forever changing. Relying on this graph would not give justifiable control results.

The second problem with plotting such a relation was the nature of data that the light sensors were gathering. The light sensors were all placed inside the cabin, and the values obtained were the luminance value after the external light passed through the window. This means that if the current DGI value was 30 , the window started dimming since the required tint according to the graph was $100 \%$. Accordingly, the measured DGI values would start to drop. Once the DGI value dropped to say 25 , the system would recognize $50 \%$ tint as the target which contradicted with the original target tint of $100 \%$. As the system kept running, the target values would be constantly changing and oscillating. There would be two possible results: 1) the window tint would keep oscillating and never stay at any constant tint value, and 2) since the window reacted very slowly compared to the ever-changing target values, eventually the window tint would settle down at a tint state where the target values do not oscillate as much and also where the window tint could not catch up with the oscillation frequency. The settling point would not represent that the user was satisfied with the tint state. This exposes another issue there was no actual set point or settling point. The user was unable to specify which level of DGI 
scale was actually appropriate for oneself. Therefore, the entire system could only behave on its own.

\subsection{Initial Fuzzy Logic Controller Design}

The objective in this thesis is to design a controller that can allow the window to dim or brighten up as fast as the window can inherently do, and ideally there should not be overshoot when the window transparency reaches the desired set-point. If there is, it should be within a range that the change of window tint should not be noticeable by the user at all.

A few participants, age between 19 to 54 at the time of experiment, were asked to sit in front of the window and notice the change of window tint. They were asked to signal when they started to notice the slightest change. The window was then slowly dimmed by performing a manual control using the RQ controller and with the light sensors in place to detect the DGI values. It was concluded that any tint changes that was within 1 unit of DGI difference was not noticeable by the participants. This result is regarded as the range of allowable overshoot and if there is any oscillation, this range would be regarded as negligible. In addition, by performing multiple tests with two light bulbs as external source, the maximum allowable time for window to dim from its clearest state is $90 \mathrm{~s}$, while to brighten up from the darkest stage is $200 \mathrm{~s}$.

Although the method proposed in the previous section was not practical, it did provide an insight of what was required in order to achieve automation for adjusting window transparency based on DGI detection. The first adjustment towards the design was to change the output.

The linguistic rule proposed was "IF DGI is...(comfort level), THEN tint\% should be...(transparency level)". Now with the modification, the proposed linguistic rule is "IF DGI is...(comfort level), THEN the window should ...(Do Nothing/Dim/Brighten Up)". The idea of 
such is that DGI represents different levels of comfort. At the range that the user feels comfortable, the window should not change the current tint. The following preliminary fuzzy controller was developed. Due to lack of initial data, the construction of it was completely based on stipulation, with the input membership functions (IMFs) similar to that of Table 2.1. Minor adjustment was made to the classification of MFs because initially the controller was tested with 5 output membership functions (OMFs) and 5 IMFs.

Figure 4.4 shows the control diagram of the fuzzy controller design, where the classification of various comfort levels served as the inputs to the fuzzy logic controller. $\Delta \mathrm{V}$ is the change in voltage required. When added with the current applied voltage $V_{0}$, the final next voltage $\mathrm{V}$ would be applied towards the window from the RQ controller. Finally, the light sensors would detect the DGI value of the window to close the control loop.

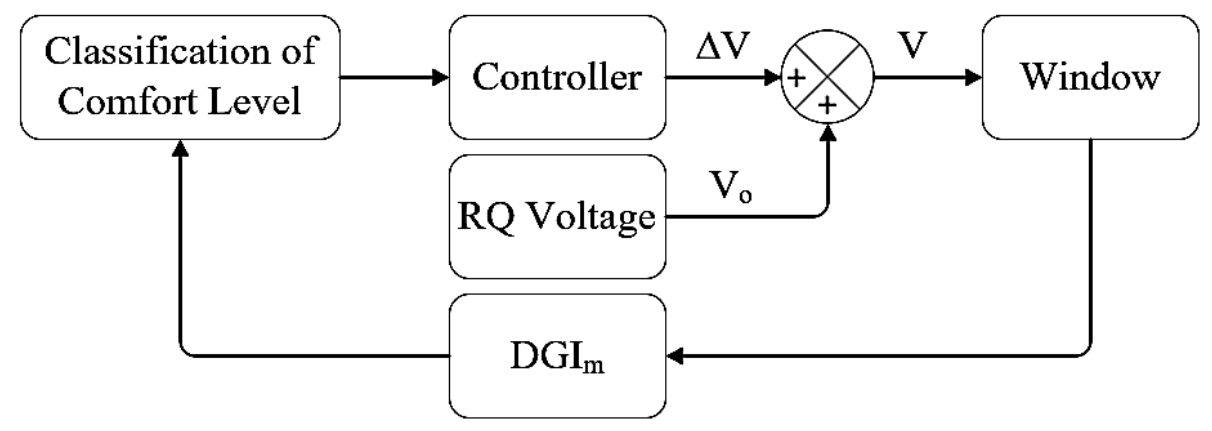

Figure 4.4 Control diagram with version 1 fuzzy logic controller - pure linguistic approach

Triangle MFs were chosen first for the preliminary design to obtain a basic idea of how well the controller would perform. Different shapes of MFs give various output values, and based on observations, triangular MFs gave more discrete output values compared to Gaussian/Z-shaped/S-shaped MFs. At the preliminary stage, triangular MFs were easier to work with, and the goal of achieving objectives stated earlier were less unaffected by shapes of MFs. 


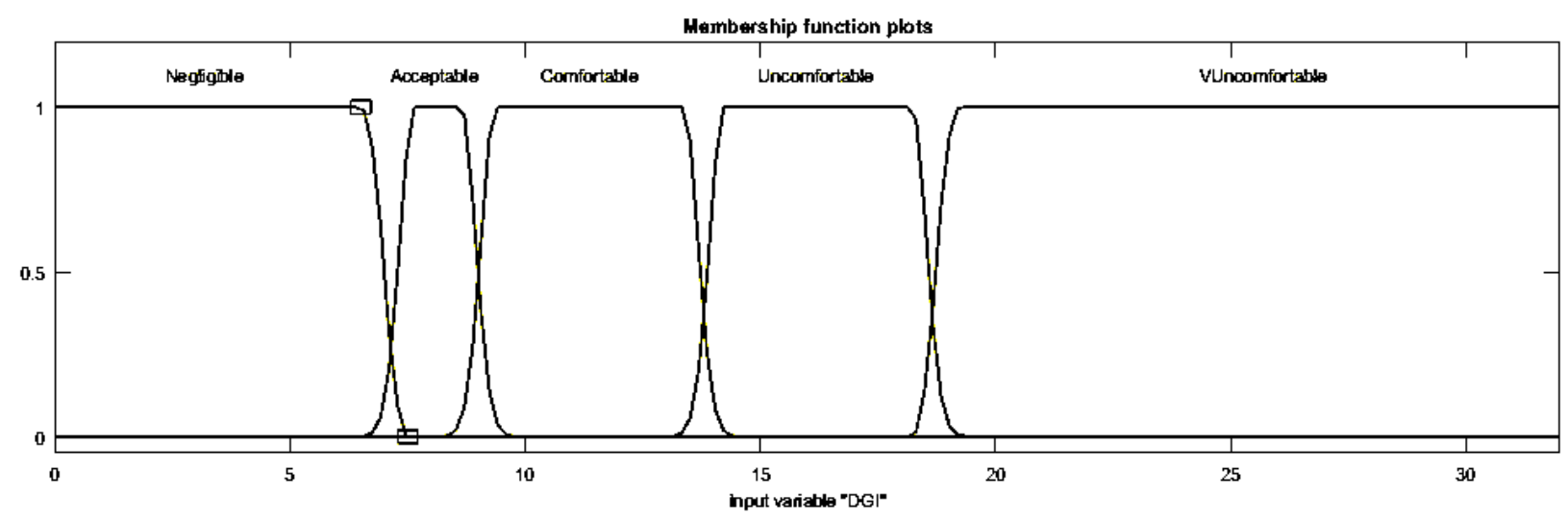

Figure 4.5 Input membership functions of version 1 fuzzy logic controller - Test 1

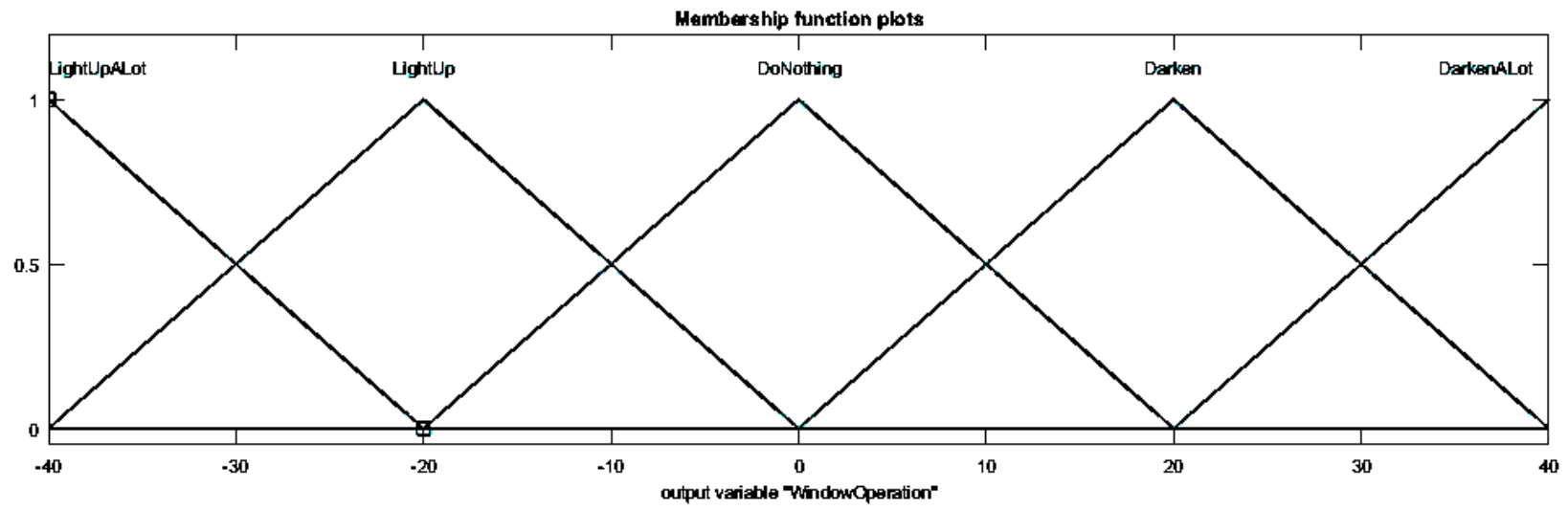

Figure 4.6 Output membership functions of version 1 fuzzy logic controller - Test 1

Gaussian combination membership function is expressed as:

$$
f(x ; \sigma, c)=\left\{\begin{array}{cc}
e^{\frac{-\left(x-c_{1}\right)^{2}}{2 \sigma_{1}{ }^{2}}}, & x \leq a \\
1, & a \leq x \leq b \\
e^{\frac{-\left(x-c_{2}\right)^{2}}{2 \sigma_{2}{ }^{2}}}, & b \leq x
\end{array}\right.
$$


Z-shaped membership function is expressed as:

$$
f(x ; a, b)=\left\{\begin{array}{cc}
1, & x \leq a \\
1-2\left(\frac{x-a}{b-a}\right)^{2}, & a \leq x \leq \frac{a+b}{2} \\
2\left(\frac{x-b}{b-a}\right)^{2}, & \frac{a+b}{2} \leq x \leq b \\
0, & x \geq b
\end{array}\right.
$$

S-shaped membership function is expressed as:

$$
f(x ; a, b)=\left\{\begin{array}{ccrl}
0, & x & \leq a \\
2\left(\frac{x-a}{b-a}\right)^{2}, & & a \leq x \leq \frac{a+b}{2} \\
1-2\left(\frac{x-b}{b-a}\right)^{2}, & \frac{a+b}{2} & \leq x \leq b \\
1, & x & \geq b
\end{array}\right.
$$

Where $a$ and $b$ are the boundaries of each segment of each membership function.

The output variable is called "Window Operation", which means the actions of the window should either dim, light up, or do nothing (remain present tint). It was known that the range of RQ values available for use on electrochromic window was -150 to 150 (corresponding to $-1.2 \mathrm{~V}$ to $1.2 \mathrm{~V}$ ). If the output range was set between these values, the window would react swiftly and as a result there would be overshoot and oscillation of window could be foreseeable. Therefore, the output range was first chosen to be a smaller range -40 to 40 for initial testing. The current goal is to achieve a DGI range from 10 to 14 (comfortable range).

Figure 4.7 shows the result of using the designed controller. With such design, the window dimmed and lit up continuously with no sign of settling at all. This means that the output range was still too large. The range of the output values were experimented with a small range (7 to 7). There was still a large overshoot, but then the window transparency settled at the value 
determined from the design of controller (Figure 4.8). If the output range was even smaller, then there would be a situation with no overshoot but a long settling time.

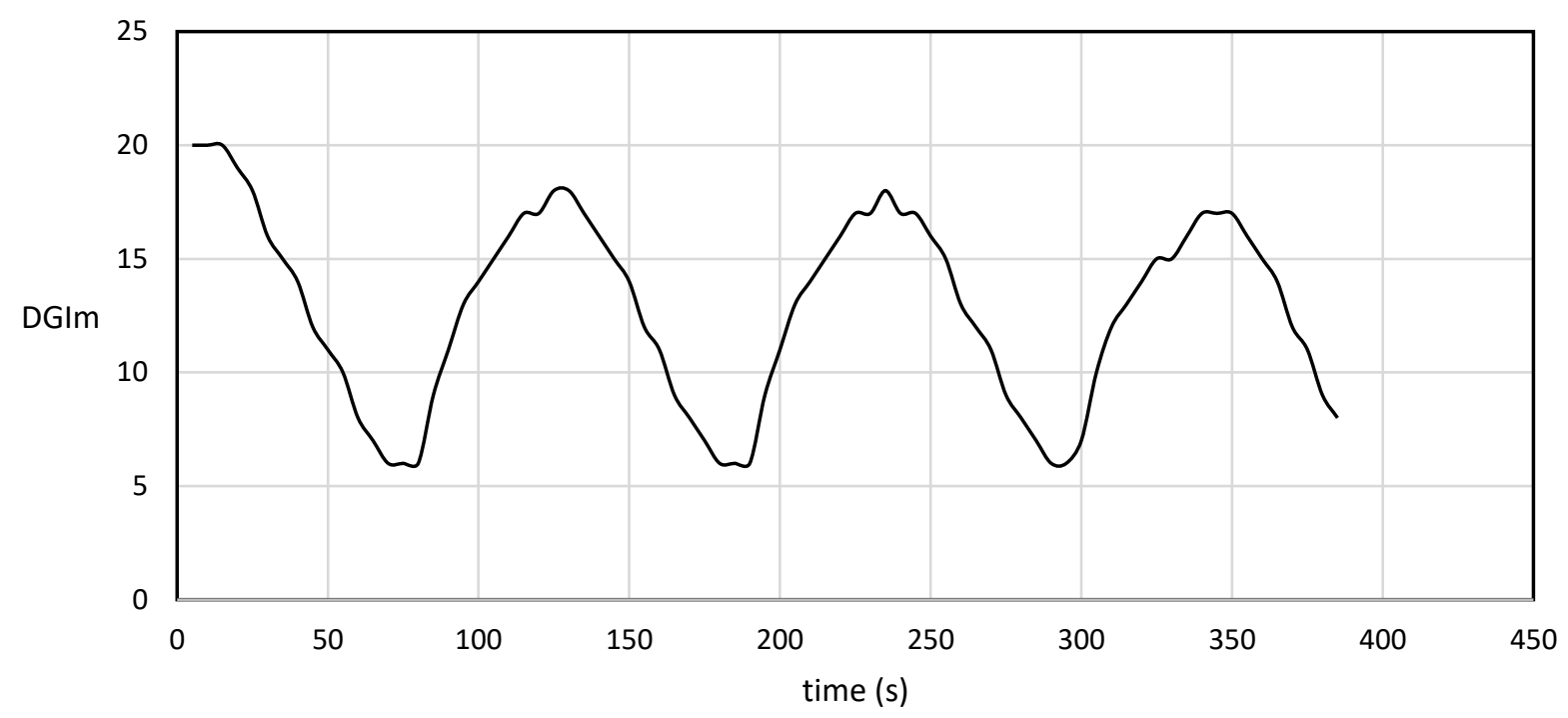

Figure 4.7 Window performance - Test 1

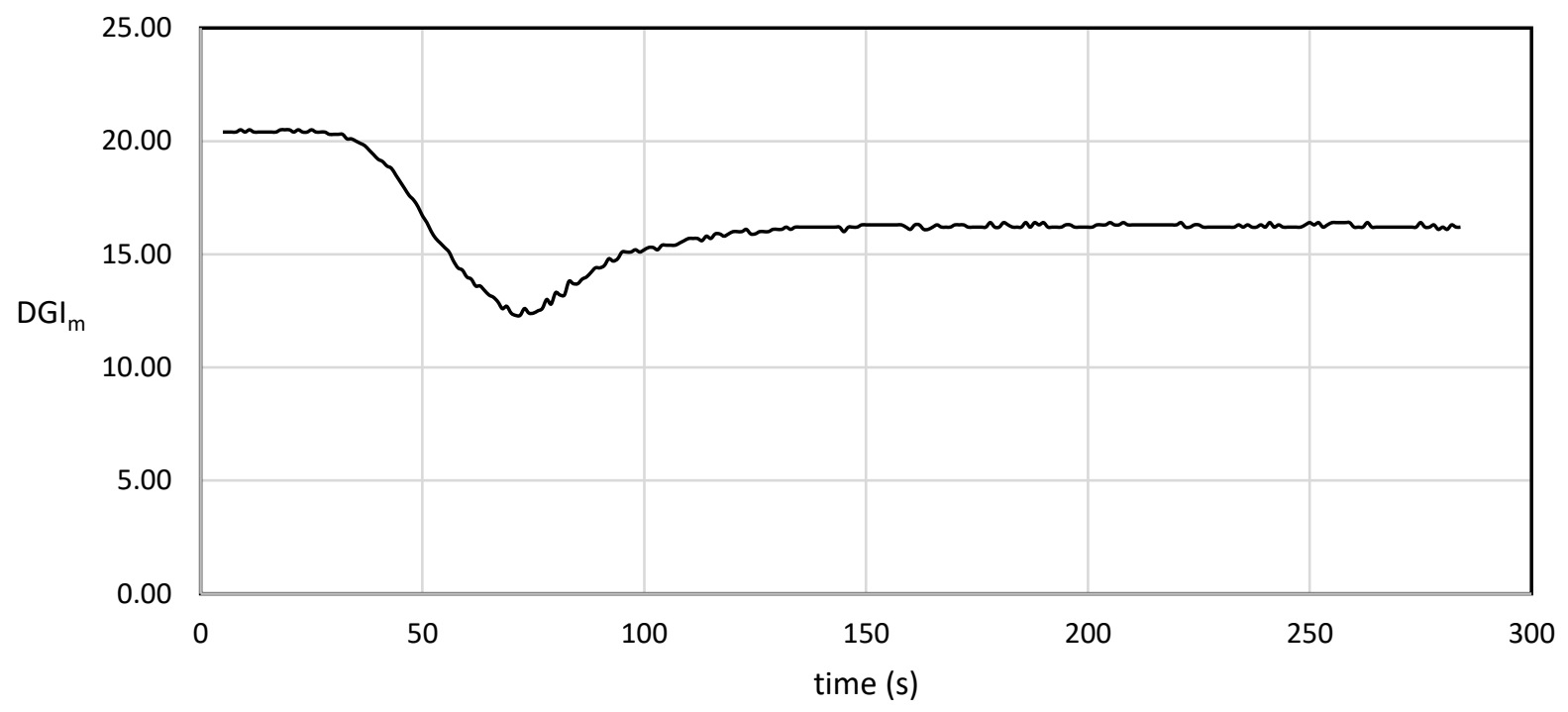

Figure 4.8 Window performance - Test 2

During the experiments, it was found that it was important to take note of the surface diagram (control surface). It plots out all the inputs and corresponding outputs (Figure 4.9). The 
more linear the relationship, the closer to the desired result, especially when the error was close to zero. This finding is important in developing the final version of the controller.

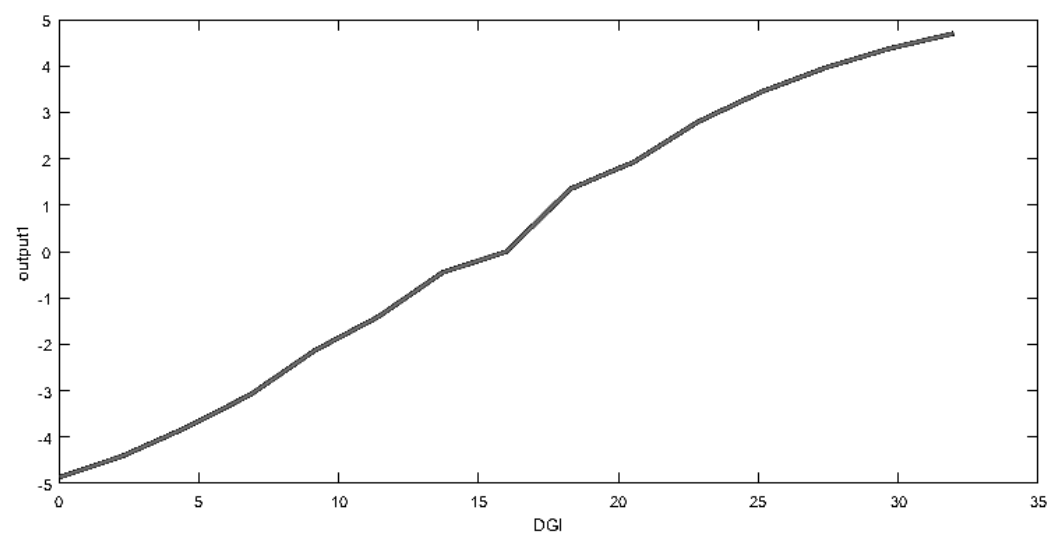

Figure $4.9 \Delta$ V output versus DGI

Through the surface diagram, it is also easy to understand that even though the midpoint of the IMF "comfortable" is 15 , the DGI settled at 16 instead. Through all the changes of IMF (Figure 4.10) and OMF (Figure 4.11) with the near linear relationship developed, when DGI was at 16 , the output of the controller was 0 . Furthermore, the "Do Nothing" output range had to be narrowed down to ensure that once the "set point" was reached, the window transparency could remain stable.

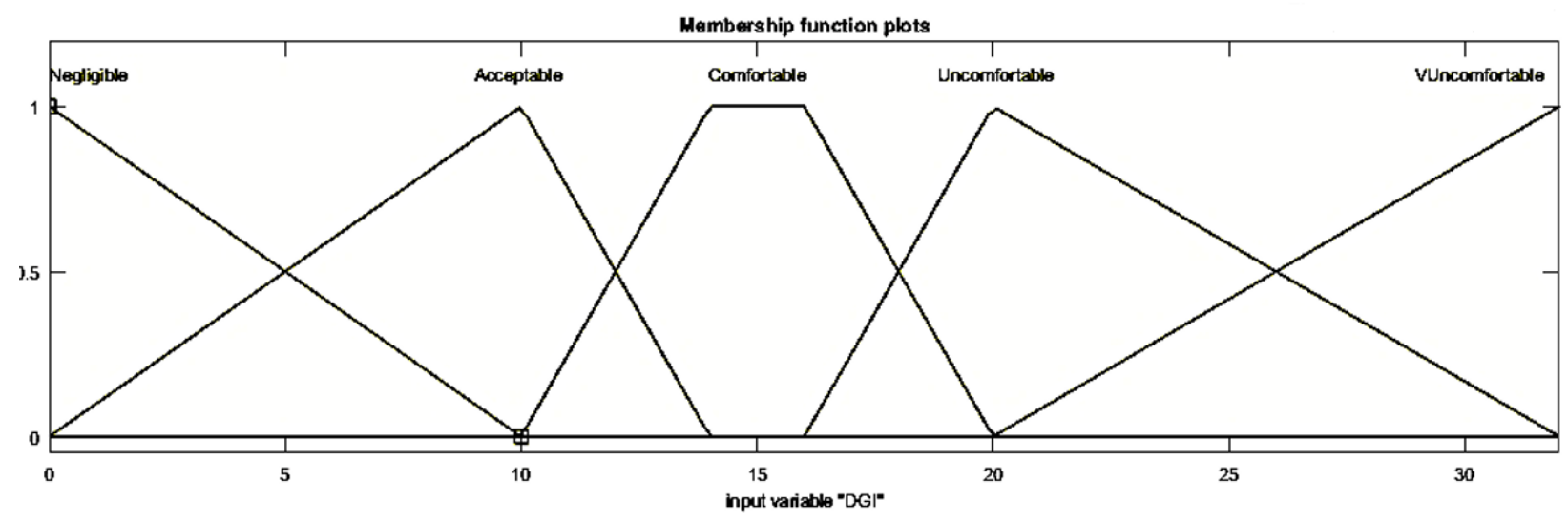

Figure 4.10 Input membership functions of version 2 fuzzy logic controller - Test 2 


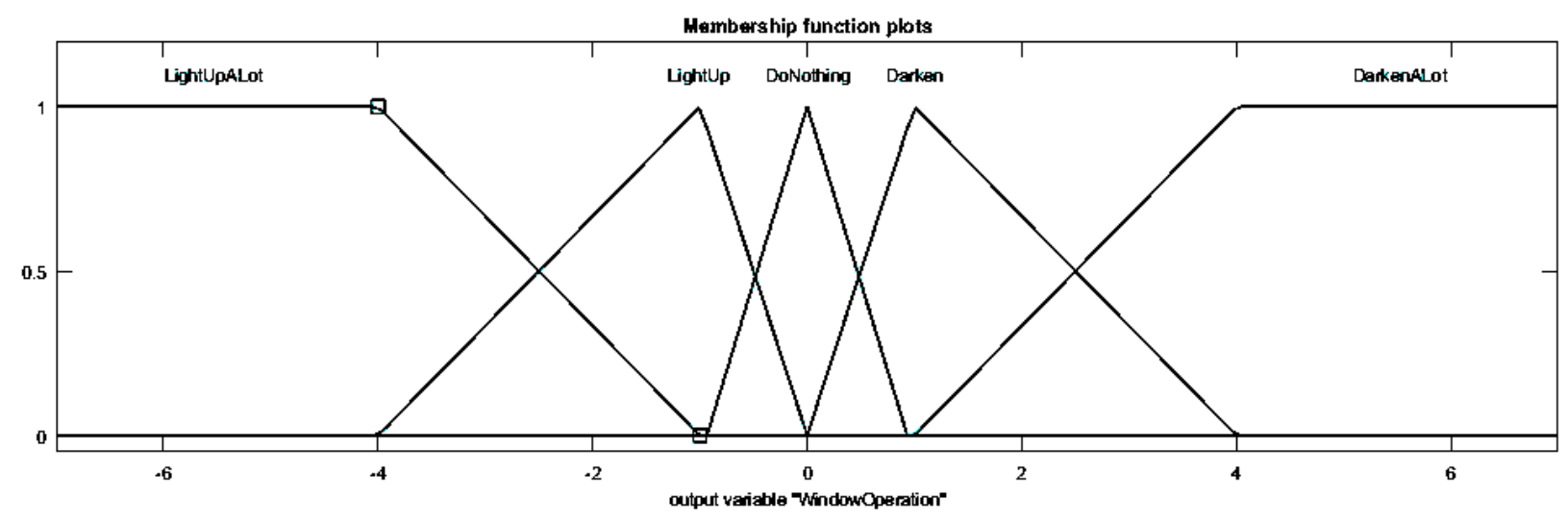

Figure 4.11 Output membership functions of version 2 fuzzy logic controller - Test 2

\subsection{Improved Fuzzy Logic Controller Design}

Through the first version of controller, it was realized that the initially proposed logic was more of linguistic description of the window operation. In reality, it is not practical to design such controller unless the time required for reaching the desired range is not a matter at all. Therefore, to obtain a practical controller, the rate of change of window transparency needs to be considered. Figure 4.12 shows the new control diagram with "Rate of Change" as the second input for the fuzzy logic controller. The rest of the diagram remained the same.

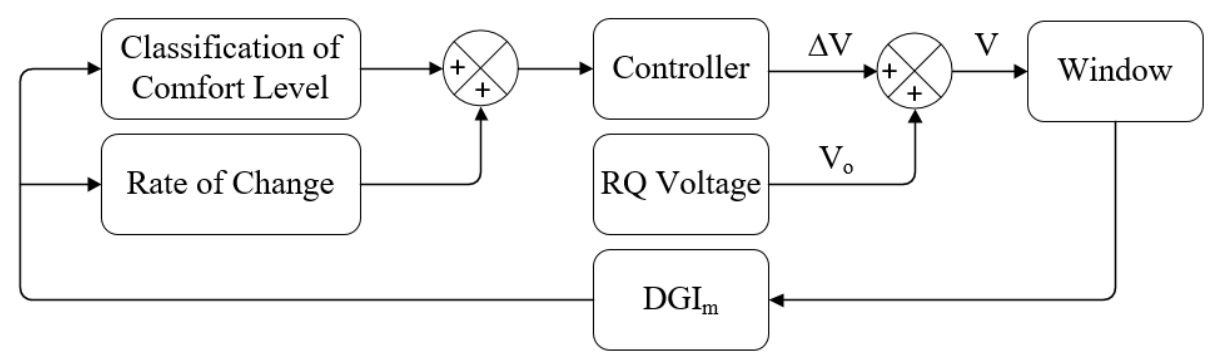

Figure 4.12 Control diagram with version 2 controller - linguistic approach with practical control method

For the same reason during the development of the first fuzzy logic controller, five MFs were established. Based on several experiments, the rate of change of DGI were recorded, and 
the suitable range of "rate of change" was determined to be within -0.4 to 0.4 (Figure 4.13), and Table 4.1 shows the fuzzy rules.

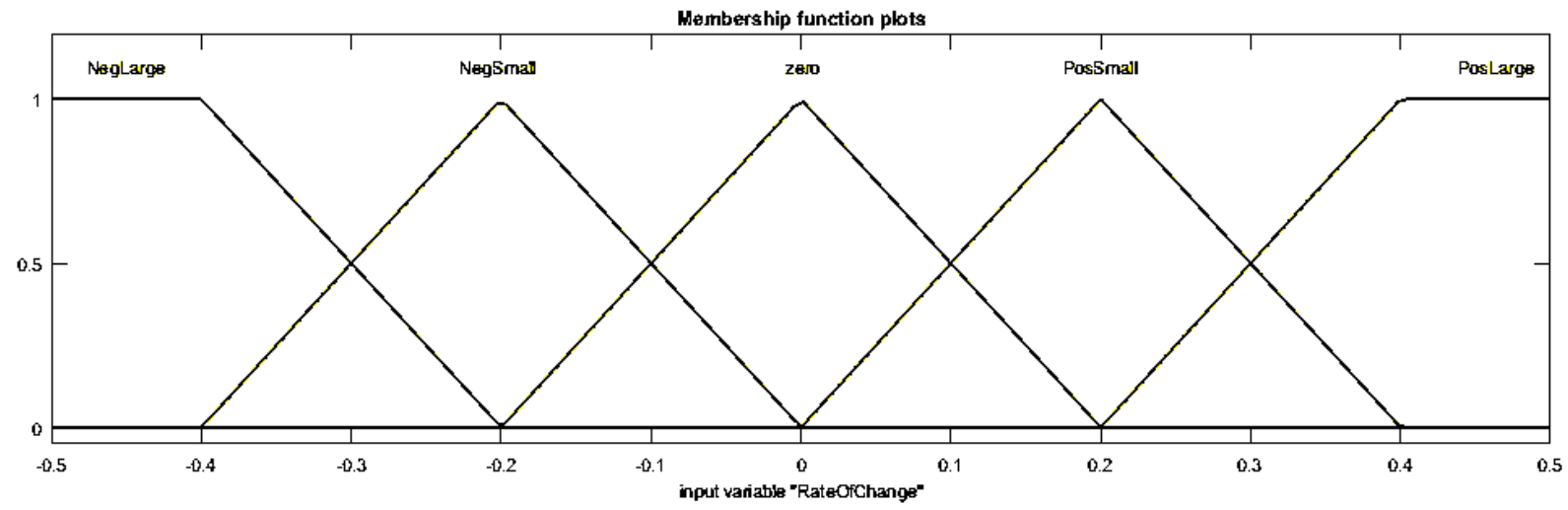

Figure 4.13 Rate of change membership functions

Table 4.1 Fuzzy rules for the improved fuzzy logic controller

\begin{tabular}{|c|l|c|c|c|c|c|}
\hline \multicolumn{2}{|c|}{} & \multicolumn{5}{c|}{ If DGI is } \\
\cline { 3 - 7 } & Negligible & Acceptable & Comfortable & Uncomfortable & $\begin{array}{c}\text { Very } \\
\text { Uncomfortable }\end{array}$ \\
\hline \multirow{3}{*}{$\begin{array}{c}\text { If } \\
\text { rate of } \\
\text { change } \\
\text { is }\end{array}$} & Negative Large & LUAL & LU & LUAL & D & DAL \\
\cline { 2 - 7 } & Negative Small & LUAL & LU & LU & D & DAL \\
\cline { 2 - 7 } & Negligible & LUAL & LU & DN & D & DAL \\
\cline { 2 - 7 } & Positive Small & LUAL & LU & D & D & DAL \\
\hline
\end{tabular}

LUAL is "Light Up a Lot", LU is "Light Up", D is "Darken", DAL is "Darken a Lot" and DN is "Do Nothing".

A test was conducted to dim the window such that the DGI maintained at 16 (Figure 4.14). The result was decent with a settling time of $42 \mathrm{~s}$. The fluctuation of DGI $\mathrm{m}_{\mathrm{m}}$ was within 1 unit. Therefore, the controller was put to an overall test (Figure 4.15) to examine the dimming and brightening up performance. 


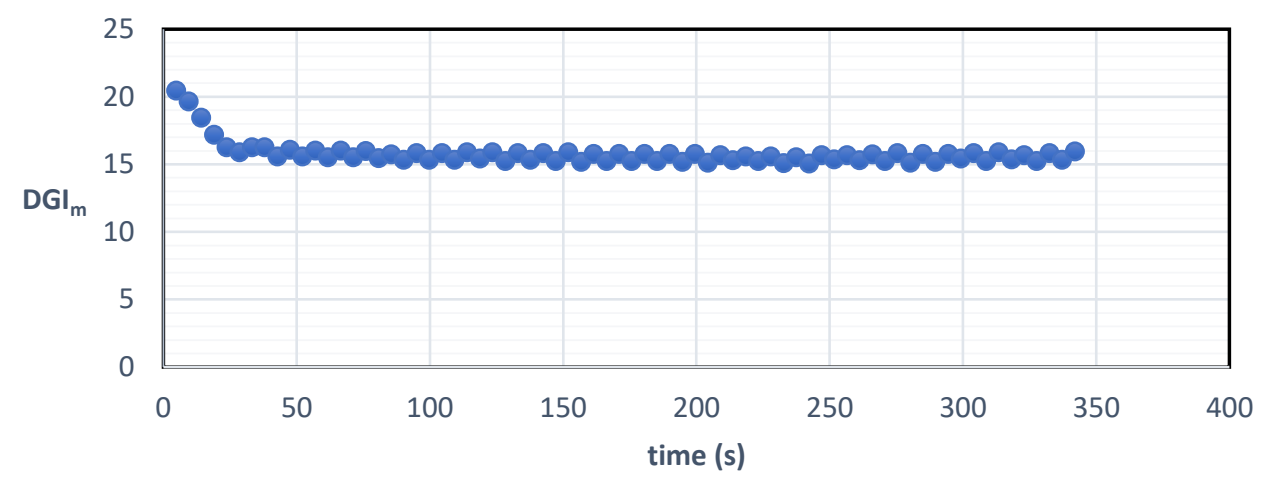

Figure 4.14 Step response of the revised fuzzy controller

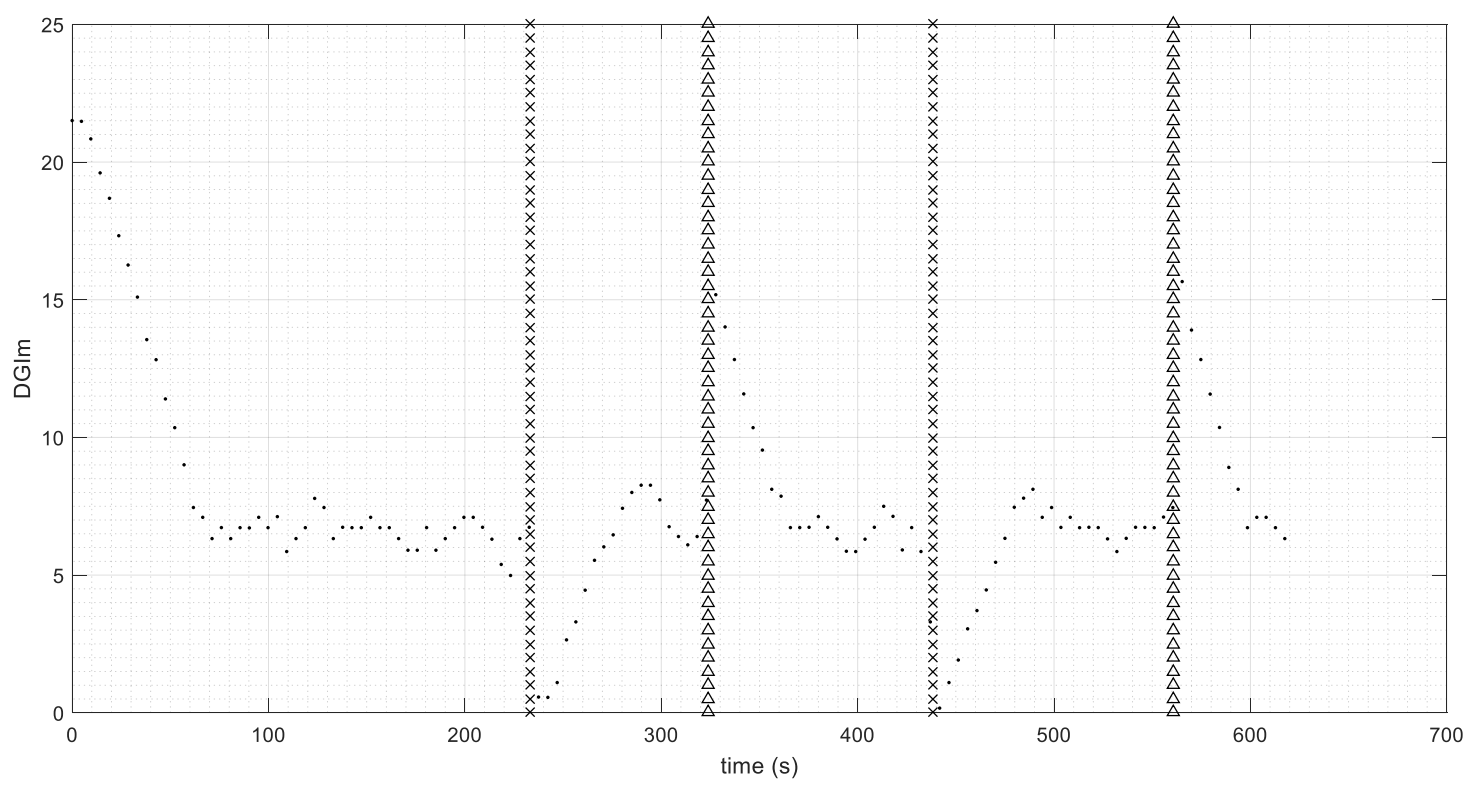

Figure 4.15 Results using the revised controller

In Figure 4.15, the four vertical lines with shapes ' $x$ ' and ' $\Delta$ ' represent the instant that the external light source was dimmed and lit up respectively, causing the detected DGI to change, and therefore the window cleared or dimmed correspondingly.

It can be seen that even though the small step response seemed to perform quite well with no overshoot and fast settling time. But when it came to more realistic testing, the controller was not able to control the window tint properly with large overshoot and inability to maintain a stable tint. The following are the reasons that caused the problems: 
1. The controller was tested with small step response, from DGI of 20 to 16 . But in the actual test, it required the DGI to be at 7 . It suggested that during the design process, a large step response should be investigated and then the small step response would be more likely to be performing well.

2. During the design of IMF, the "comfortable" range was from 3 to 5 . This means that when the $\mathrm{DGI}_{\mathrm{m}}$ reached this range, the fuzzy controller output a value that was less sensitive towards the inputs. This caused the voltage values not sufficient to dim or clear up the window quick enough to avoid overshoot.

Even though the problem of the first version of controller was solved, this controller was still problematic. Furthermore, if a similar version of controller was being used, the user would run into problem of not being able to change the desirable range of comfort since the adjustment would have to be made through the fuzzy IMFs.

\subsection{Final Fuzzy Controller Design}

With the experience learnt from previous designs, it is known that an explicit set-point needs to be implemented. The input "rate of change" remained the same, while the other input was no longer DGI $\mathrm{I}_{\mathrm{m}}$. Rather, it was changed to $\Delta \mathrm{DGI}_{\mathrm{m}}$. This control concept is a PD controller. But instead of having a constant gain value that is proportional to the error or change in error in conventional PD controllers, the gain values are inherently calculated constantly through fuzzy logic. Figure 4.16 shows the final control diagram with the required changes. Figure 4.17 shows the results of window tinting. 


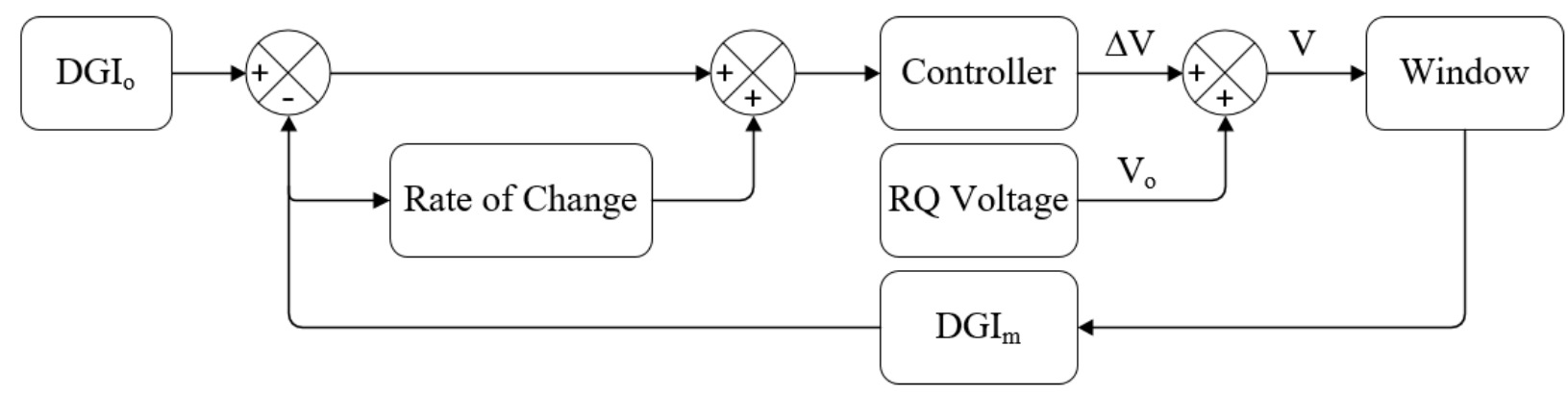

Figure 4.16 Control diagram for version 3 controller - most practical approach (PD controller)

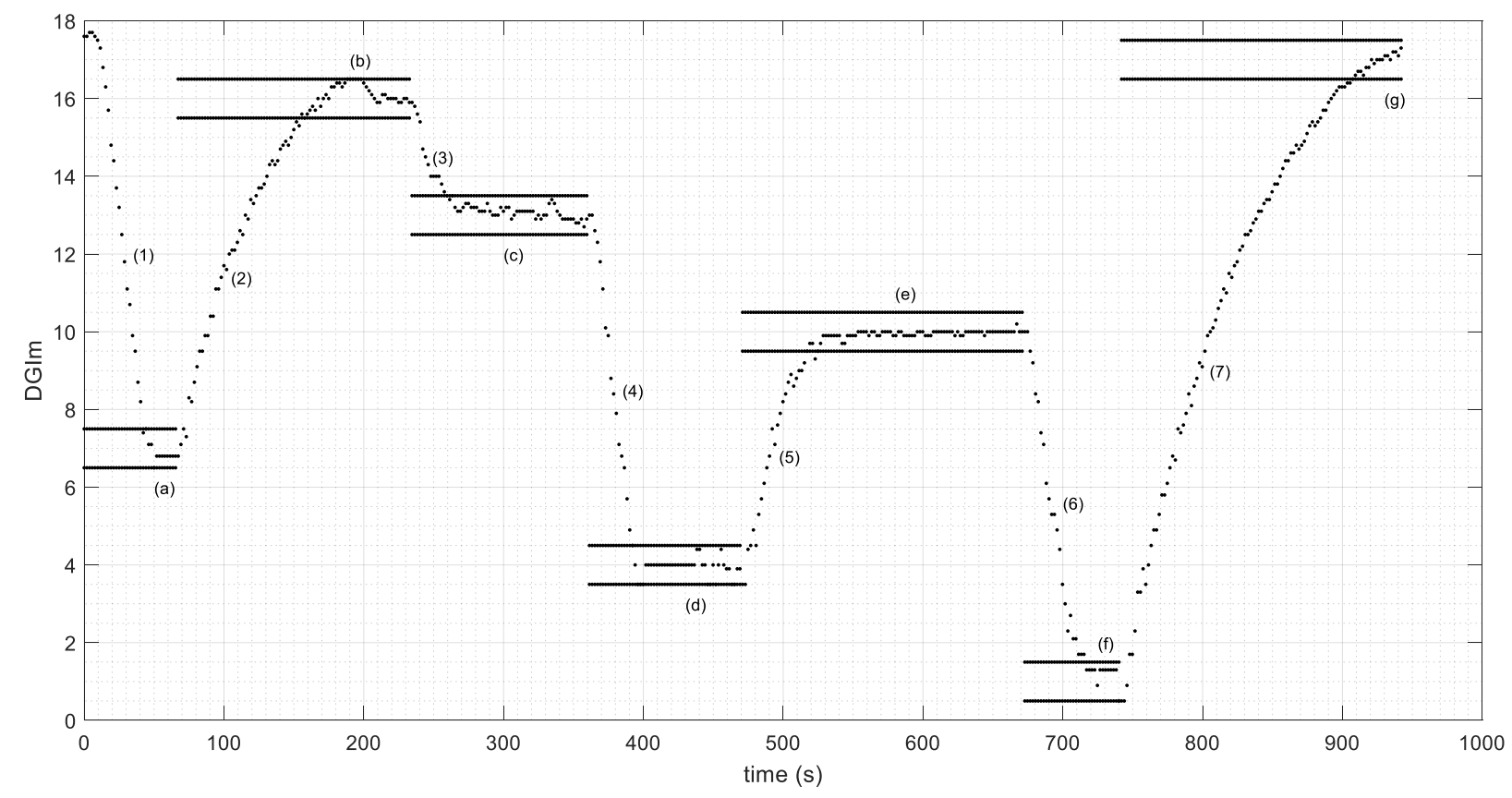

Figure 4.17 Results using final fuzzy logic controller version

There are a few things to know before understanding how the controller performed:

i. The horizontal lines represented the boundaries of acceptable overshoot and range of oscillation. It was mentioned that DGI fluctuation within 1 unit was considered negligible. The midpoint of the boundaries was from the various set-points $\mathrm{DGI}_{\mathrm{o}}$, and the boundaries were set as $\mathrm{DGI}_{0} \pm 0.5$. 
ii. A few different set-points, $\mathrm{DGI}_{\mathrm{o}}$ were demonstrated. Not only it showed the performance of the controller, but also showed that the user could easily change the desired glare level.

iii. When the window was fully clear, the DGI level was approximately 18 . When the window was fully dimmed, the DGI $\mathrm{m}$ level was approximately 1 .

iv. A dropping of $\mathrm{DGI}_{\mathrm{o}}$ means that the window shall start dimming while an increase of $\mathrm{DGI}_{0}$ means that the window shall start clearing.

v. The external light source remained constant throughout the entire experiment. Whether changing the light source illuminance or changing the set point of the desired glare level, the analysis for controller performance remains unchanged. The controller output voltage changed values but the set point was maintained throughout the entire test.

Table 4.2 shows that the transition times were all well within the requirement of $90 \mathrm{~s}$ for dimming and 200s for clearing. Table 4.3 shows that when reaching the set points there is minimal or no overshoot. From the graph, even though there were fluctuations where DGI $\mathrm{m}_{\mathrm{m}}$ was supposed to be settled, their amplitudes were well within the acceptable range. Therefore, this controller met all the requirements and shall be the final version in terms of performance for practical use. Also, Figure 4.18, Figure 4.19 and Figure 4.20 show the IMFs and OMFs used to design the fuzzy logic version of PD controller, and Figure 4.21 shows the resulting control surface diagram.

Table 4.2 Transition times for various dimming and clearing sections as shown in Figure 4.17

\begin{tabular}{|c|c|c|c|}
\hline dimming sections & transition time (s) & clearing sections & transition time (s) \\
\hline$(1)$ & 52 & $(2)$ & 143 \\
\hline$(3)$ & 32 & $(5)$ & 49 \\
\hline$(4)$ & 33 & $(7)$ & 180 \\
\hline$(6)$ & 45 & \multicolumn{2}{c}{} \\
\hline
\end{tabular}


Table 4.3 Amplitude of overshoot

\begin{tabular}{|c|c|c|c|}
\hline section & overshoot amplitude & section & overshoot amplitude \\
\hline (a) & $/$ & (e) & $/$ \\
\hline (b) & 0.5 DGI & (f) & N/A \\
\hline (c) & $/$ & (g) & \multicolumn{2}{c}{} \\
\hline (d) & $/$ & \multicolumn{2}{c}{}
\end{tabular}

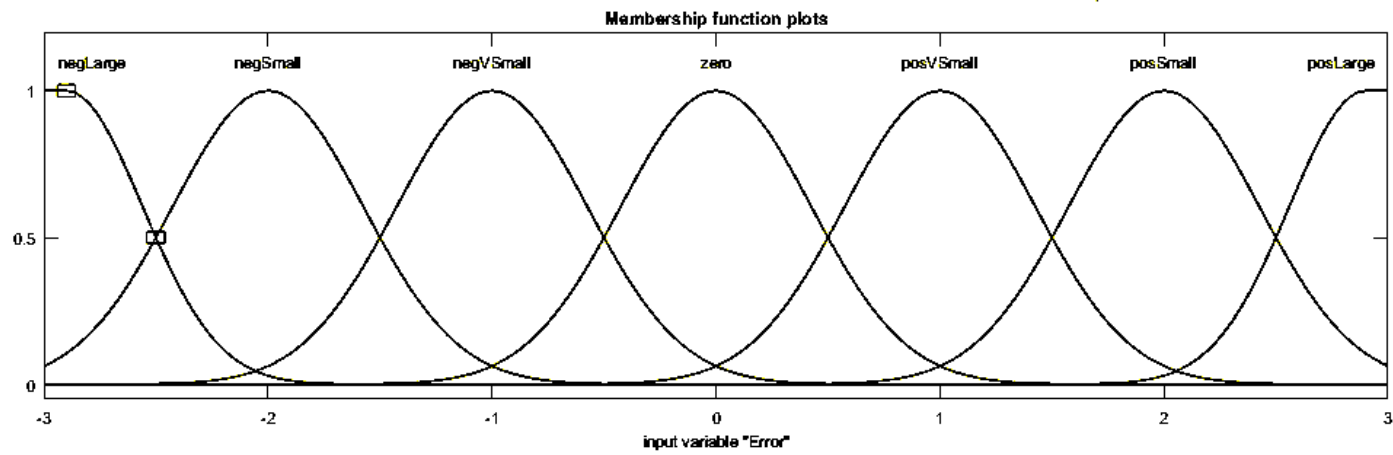

Figure 4.18 Input membership function "error" for final fuzzy logic controller

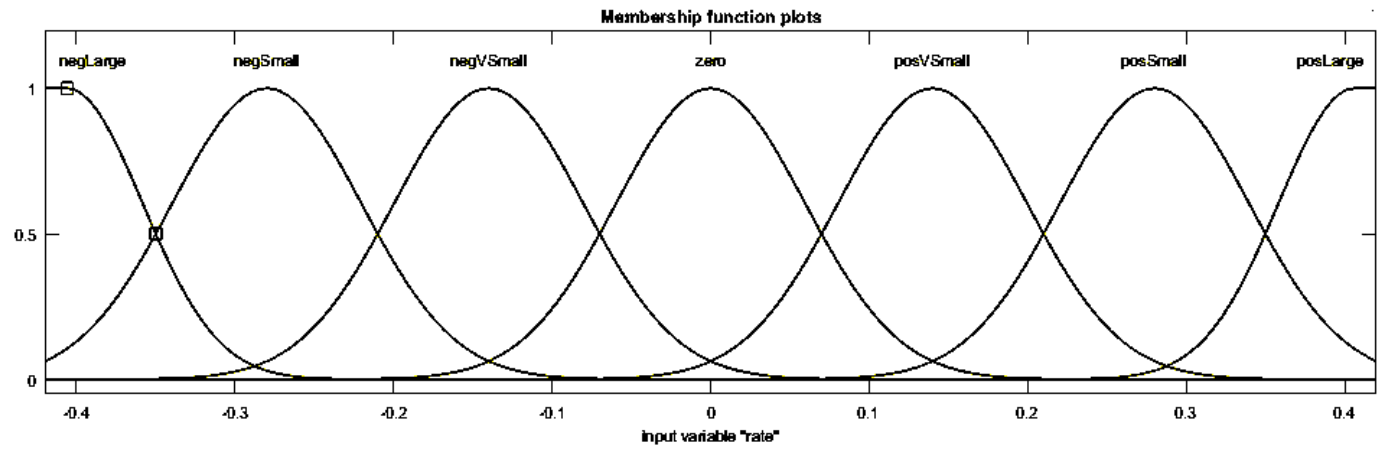

Figure 4.19 Input membership function "rate of change" for final fuzzy logic controller

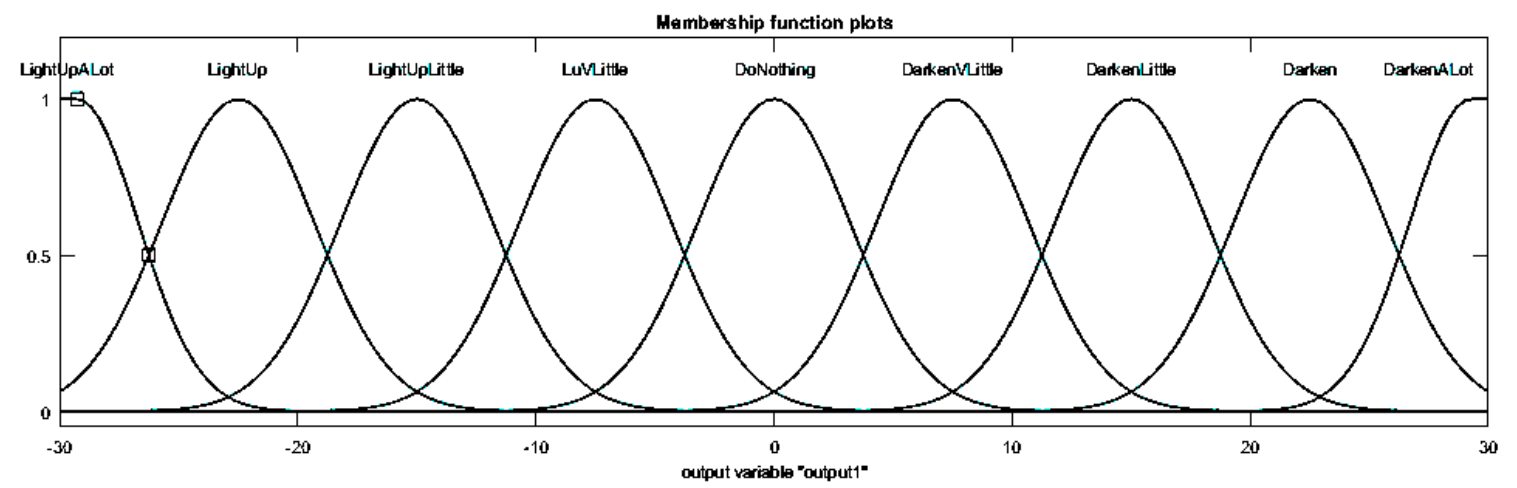

Figure 4.20 Output membership function "window operation” for final fuzzy logic controller 


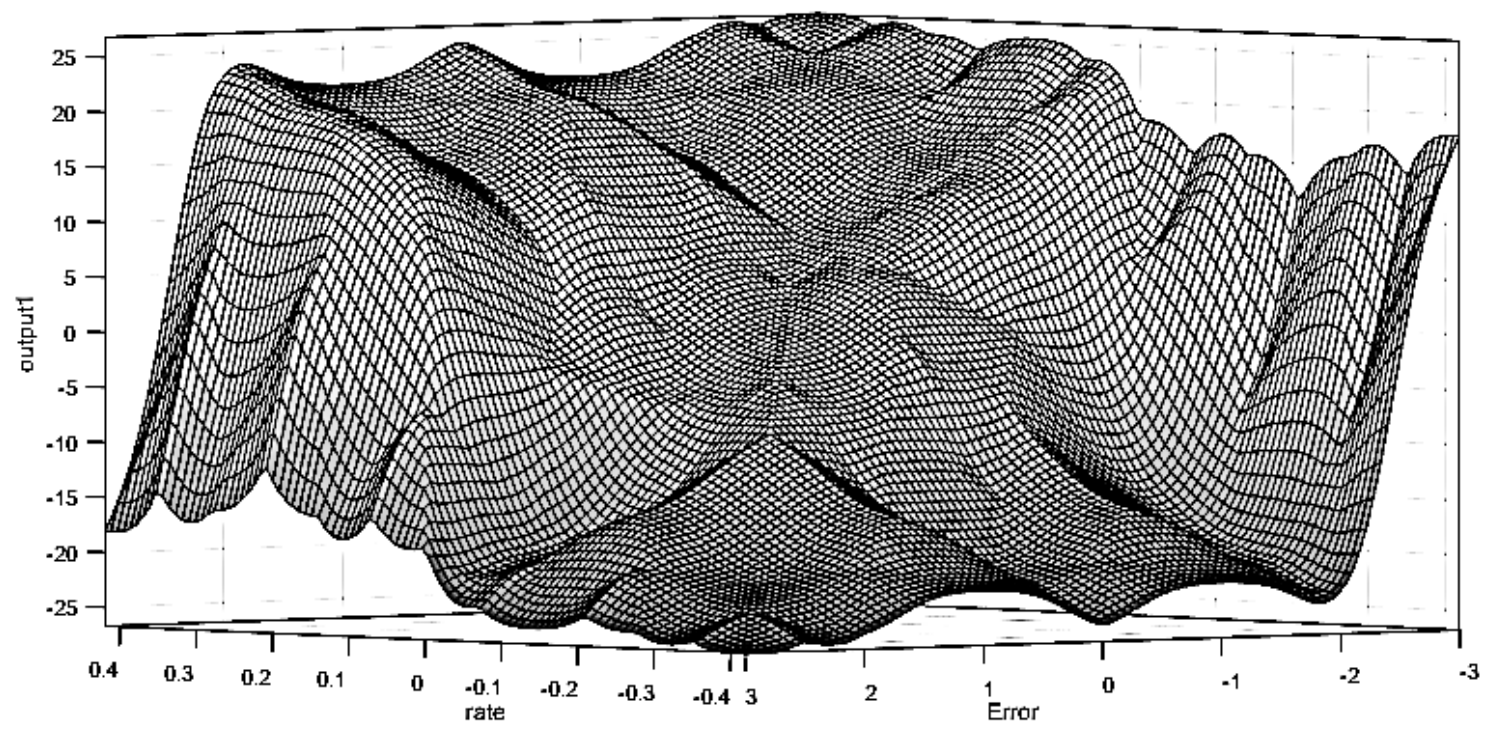

Figure 4.21 Control surface diagram of final fuzzy logic controller

Table 4.4 shows the fuzzy rules used for this controller. D stands for "darken", LU stands for "light up", AL stands for "a lot" and v stands for "very". The membership functions were completely changed compared to the previous controllers, and such design of configurations was to allow the synthesis for the desired control surface.

Table 4.4 Fuzzy rules for final fuzzy logic controller

\begin{tabular}{|c|c|c|c|c|c|c|c|c|}
\hline \multicolumn{2}{|c|}{} & \multicolumn{7}{|c|}{$\Delta$ DGI $_{\mathrm{m}}$} \\
\cline { 2 - 9 } \multicolumn{2}{|c|}{} & $\begin{array}{c}\text { Neg } \\
\text { Large }\end{array}$ & $\begin{array}{c}\text { Neg } \\
\text { Small }\end{array}$ & $\begin{array}{c}\text { Neg } \\
\text { Very Small }\end{array}$ & zero & $\begin{array}{c}\text { Pos } \\
\text { Very } \\
\text { Small }\end{array}$ & $\begin{array}{c}\text { Pos } \\
\text { Small }\end{array}$ & $\begin{array}{c}\text { Pos } \\
\text { Large }\end{array}$ \\
\hline \multirow{5}{*}{$\Delta D G I_{m}$} & neg Large & DAL & LUAL & LU & LUAL & LU & LUAL & LUAL \\
\cline { 2 - 9 } & Neg Small & DAL & LU & Lulittle & LU & Lulittle & LU & LUAL \\
\cline { 2 - 9 } & $\begin{array}{c}\text { Neg Very } \\
\text { Small }\end{array}$ & DAL & Lulittle & Luvlittle & LuVlittle & Luvlittle & Lulittle & LUAL \\
\cline { 2 - 9 } & zero & DAL & Dlittle & Dvlittle & DN & Luvlittle & LU & LUAL \\
\cline { 2 - 9 } & $\begin{array}{c}\text { Pos Very } \\
\text { Small }\end{array}$ & DAL & Dlittle & Dvlittle & Dvlittle & Dvlittle & Dlittle & LUAL \\
\cline { 2 - 9 } & Pos Small & DAL & D & Dllittle & D & Dlittle & D & LUAL \\
\cline { 2 - 9 } & Pos Large & DAL & DAL & D & DAL & D & DAL & LUAL \\
\hline
\end{tabular}


The control surface diagram can be split into five main sections (Figure 4.22) to understand how the controller performed according to the fuzzy rules by correlating it with the results shown in Figure 4.17.

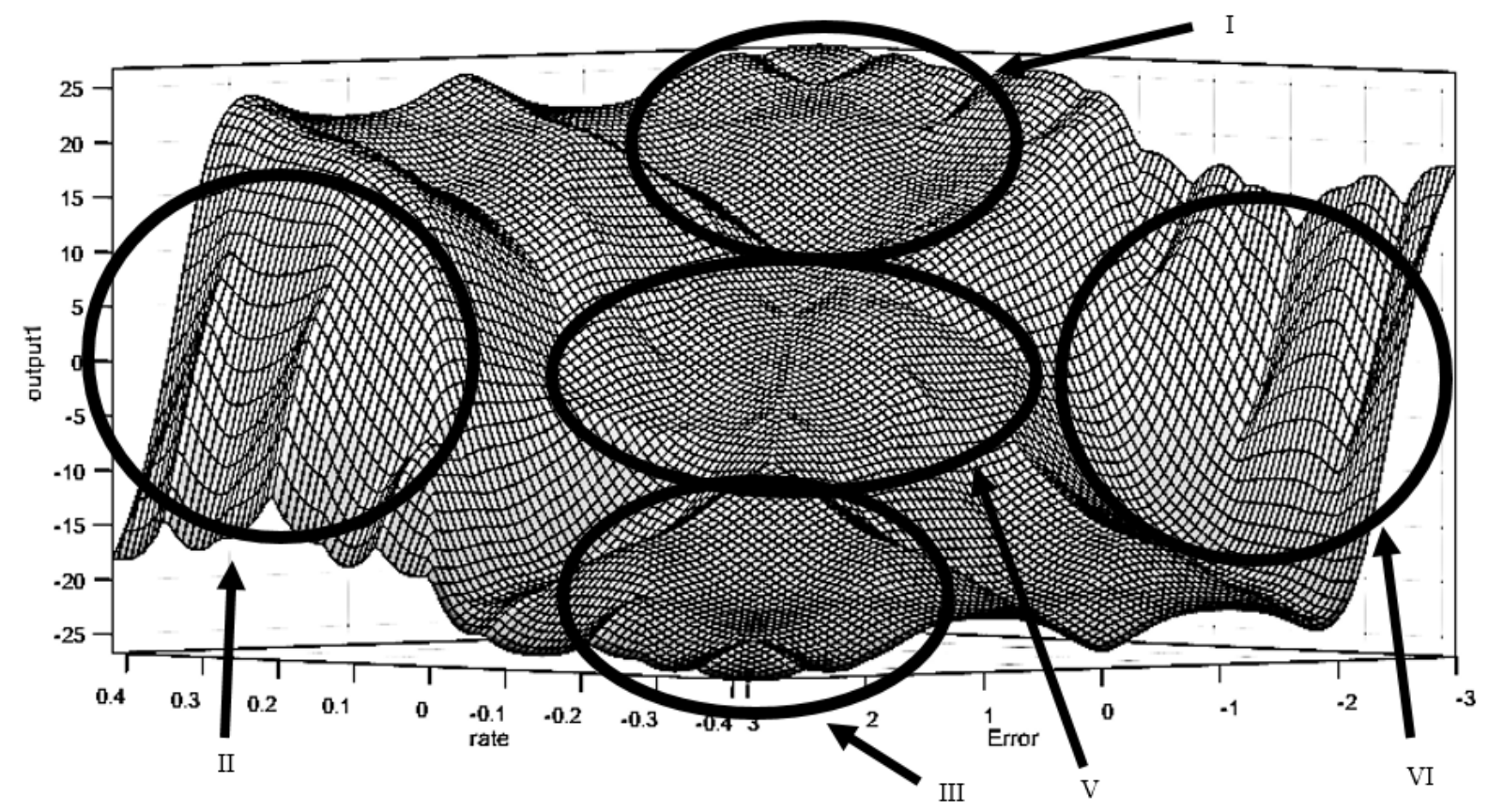

Figure 4.22 Control surface diagram categorized into five sections

Section I \& III (representing (i) in Figure 4.23): These two sections are the extreme areas where the error compared to the set point was large. Regardless of what the rate of change was, the controller still needed to output a large value to reduce the error - a negative value to clear the window and a positive value to dim the window.

Section II and VI (representing (ii) in Figure 4.23): The bottom part of section deals with the scenario where the error was gradually getting less positive, meaning that the window turning clear and approaching to the set point, but the rate of change was still largely positive. In this case, the window should receive a lower voltage value to slow down the clearing process. Then the diagram jumps up to positive values as the error approaches zero. When the rate of error was 
still positive (which means clearing quickly), a dimming value needed to be added to bring down the rate. Ideally As the error gets to zero, the rate should be close to zero. By observing horizontally within the section, the closer the rate is to zero, the lower the magnitude of "the jump". Section IV is the exact opposite of section II, which deals with quick window darkening. When the error approached zero, a negative value was added to bring the rate slower to approach to zero.

Section V (representing (iii) in Figure 4.23): This section of control surface is responsible for maintaining a constant $\mathrm{DGI}_{\mathrm{m}}$ level as stable as possible. It was designed such that at zero error and zero rate of change, the output $(\Delta \mathrm{V})$ was exactly zero. At the areas that the error or rate of change is slightly deviated from zero, the control surface is slightly concave inwards. This is similar to the scenario that a rolling ball tries to settle at the bottom of a trough, but the slope around this area was designed to be very gentle such that the output values were very small to minimize the unwanted fluctuations. Ideally this area should be flat but if it was completely flat, it would pose the problem of irresponsiveness to small changes. However, having this compromise to sacrifice how well the systems settled at the set point, this explains why there are some small fluctuations, but the amplitudes were too small to affect our eyes. Therefore, the slope demonstrated in the control surface is acceptable.

Two other major changes made to the development of final controller were the shape of membership functions and also the number of membership functions. Figure 4.24 shows the results of using a fuzzy controller with five IMFs and OMFs with triangular shaped membership functions. It can be seen that such controller only satisfied the settling time. It was not able to maintain the DGI $\mathrm{m}_{\mathrm{m}}$ within the acceptable region of oscillation, and there are offsets of 0.5 DGI. It can also be noticed that the fluctuation of $\mathrm{DGI}_{\mathrm{m}}$ at the settled state was quite rigorous. 


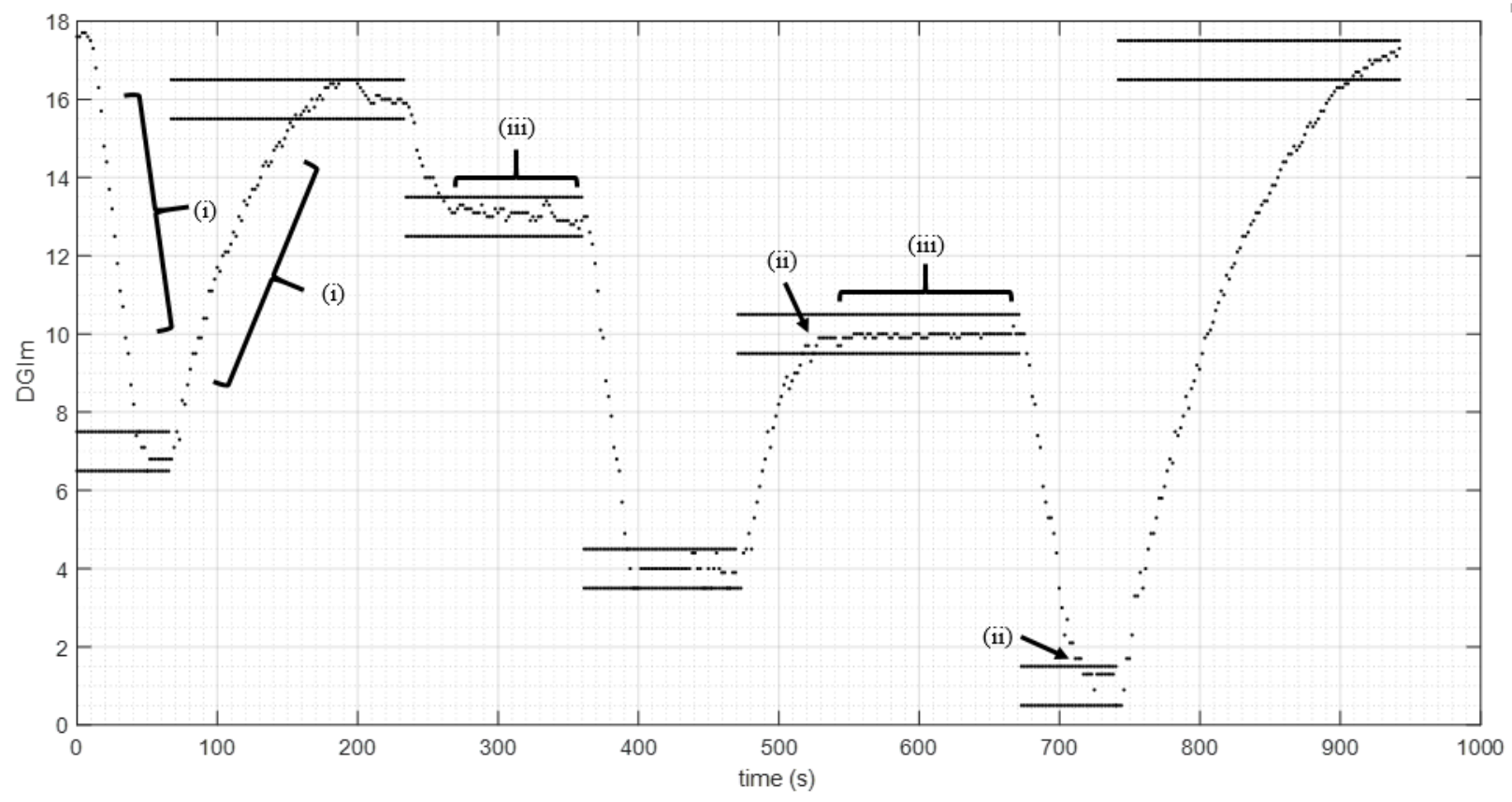

Figure 4.23 Co-relation of result with the control surface

This means the controller was attempting to correct every little change in $\mathrm{DGI}_{\mathrm{m}}$ value by outputting a $\Delta \mathrm{V}$ value that was just too large pushing the DGI into the other direction. This can be explained by the lack of gentleness and smoothness in section $\mathrm{V}$ of control surface discussed in Figure 4.22 earlier. To solve this settling problem two more IMFs and OMFs were added explaining the increased number of MFs in the final design.

Now that the reason behind seven IMFs was explained, the last issue to discuss is why Gaussian shaped MFs were used instead of triangular ones. The advantage of using Gaussian MFs can be seen by comparing Figure 4.24 and Figure 4.25 . Both with the same number and similar configuration of MFs. Using Gaussian MFs yielded a much smoother DGI $\mathrm{m}_{\mathrm{m}}$ control especially during the state that the controller needs to maintain $\mathrm{DGI}_{\mathrm{m}}$ at the set-point. The reason behind such difference was because of the defuzzification method that was used called centre of 
gravity method. This method was to find the centroid of the total area under the triggered membership functions by the input to the corresponding output inferred by the fuzzy rules.

$$
\text { Output result }=\frac{\sum_{n=A}^{n=I}\left[\left(F O_{n}\right)\left(\text { FGrade }_{n}\right)\right]}{\sum_{n=A}^{n=I} \text { FGrade }_{n}}
$$

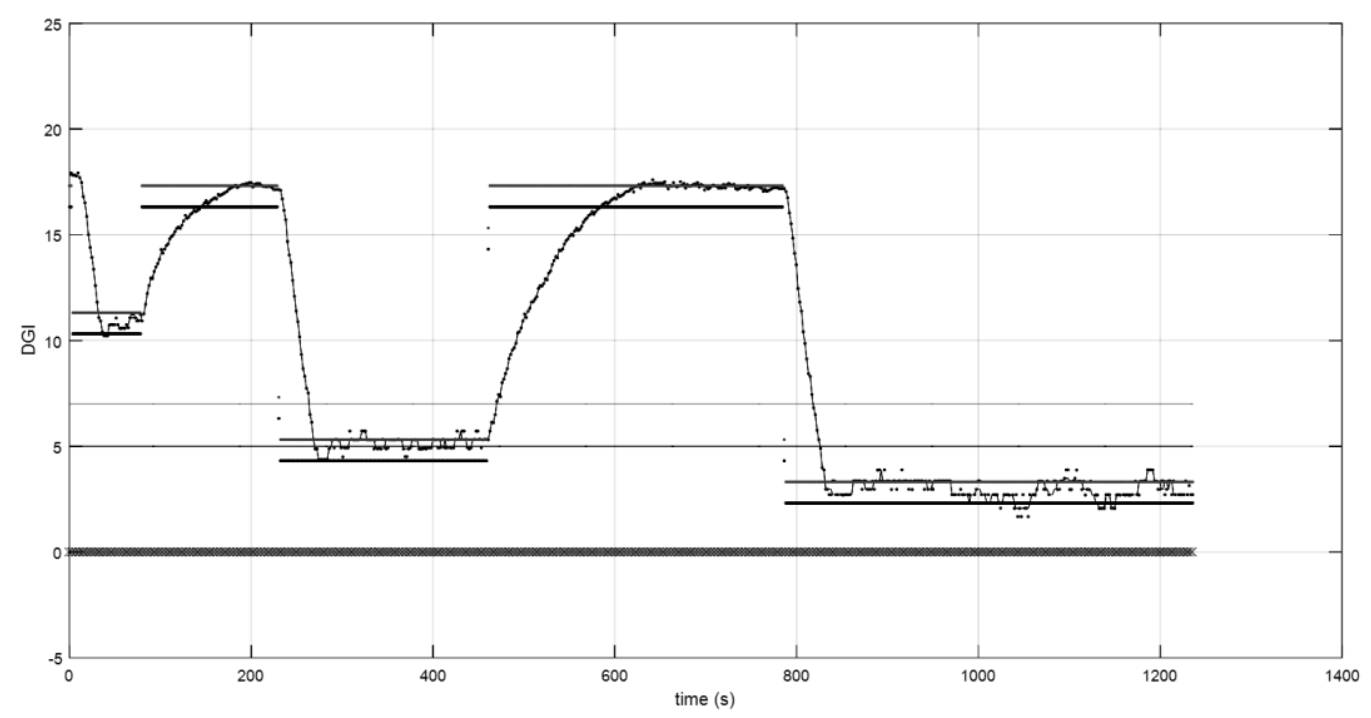

Figure 4.24 Results of using fuzzy logic controller with 25 rules and triangular shaped membership functions

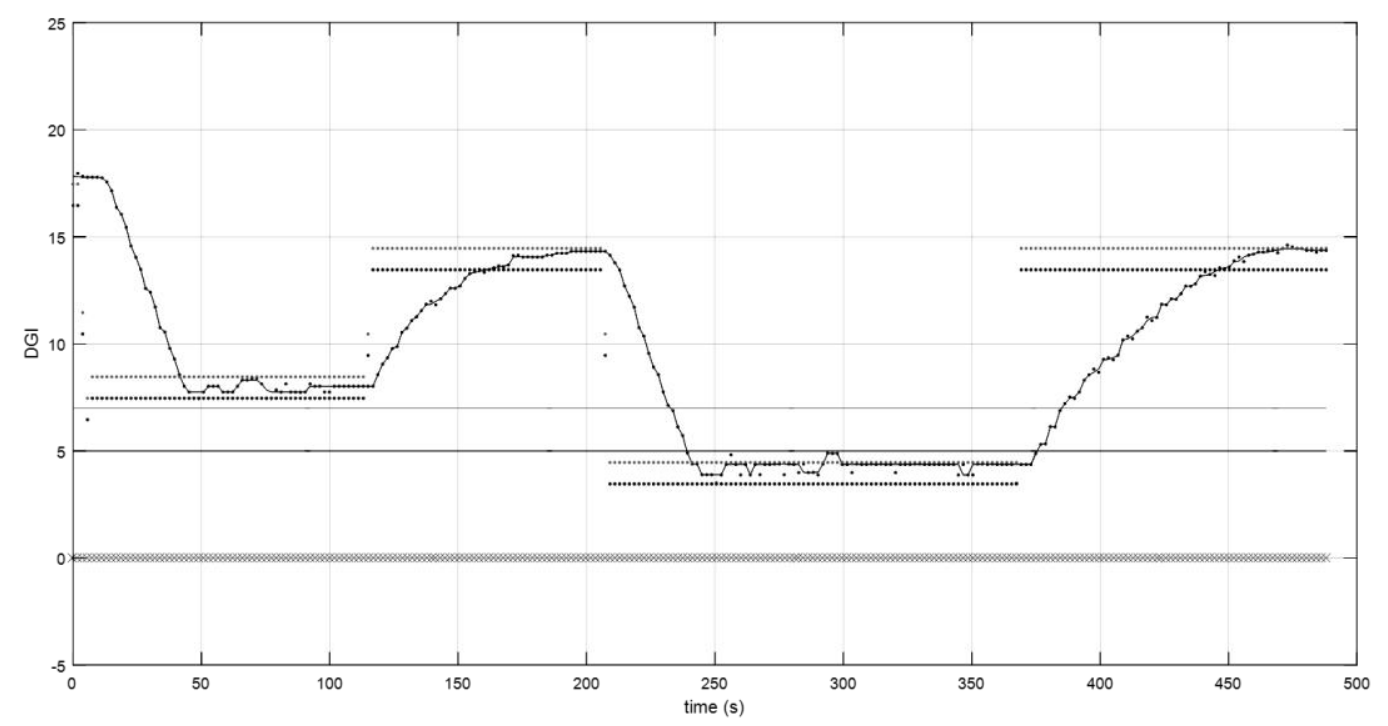

Figure 4.25 Results of using fuzzy logic controller with 25 rules and Gaussian shaped membership functions 
The following demonstration further elaborates on the different shapes of membership functions. It is a very simply fuzzy logic design with only 3 IMFs and OMFs to represent a portion of the designed PD controller. Figure 4.26 to Figure 4.29 show the membership functions used for demonstration.

The Gaussian membership functions are designed in a way that is identical to the ones used in the fuzzy PD controller and the triangular membership functions are designed in a way such that the output is similar to that of Gaussian. Three membership functions are required to show the transition between adjacent membership functions. The range of values replicate that of the PD controller. The actual numerical values in all the figures do not mean anything, and what is important is the shape of the output diagram.

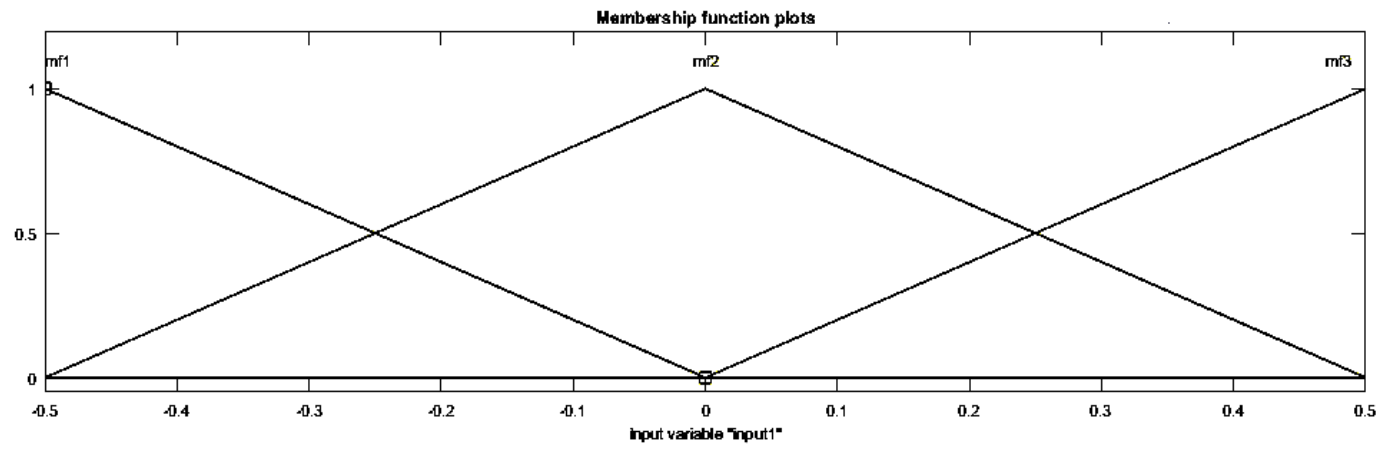

Figure 4.26 Demonstration - triangular input membership functions

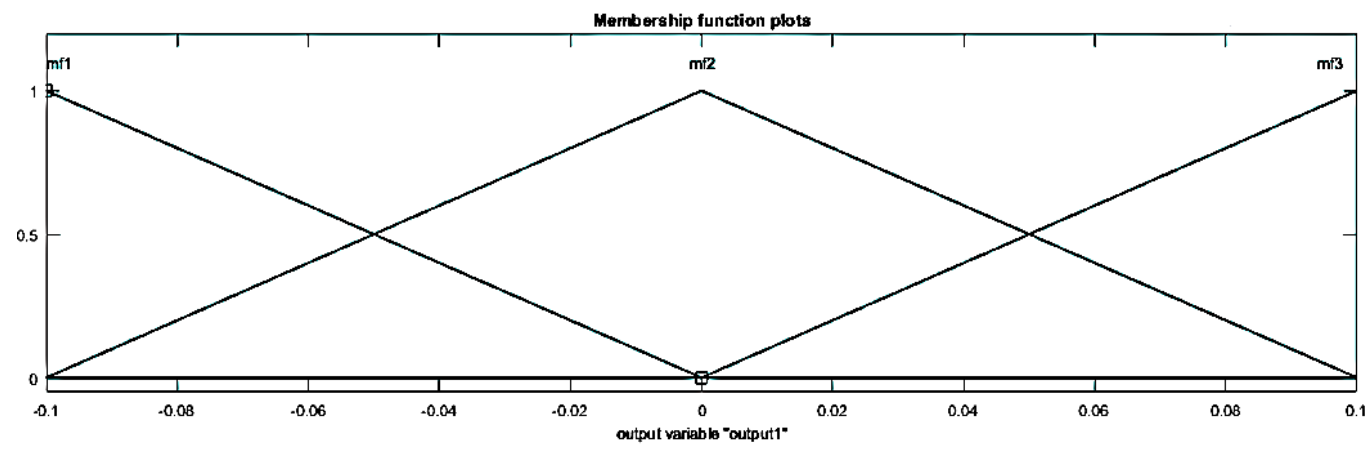

Figure 4.27 Demonstration - triangular output membership functions 


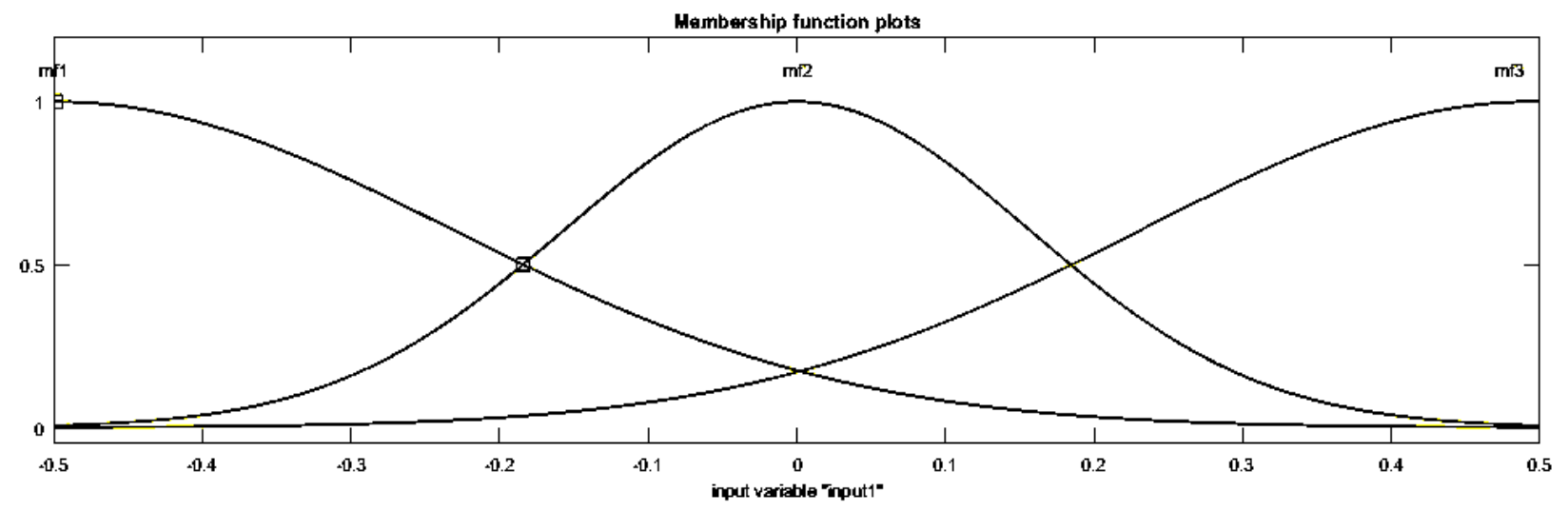

Figure 4.28 Demonstration - Gaussian input membership functions

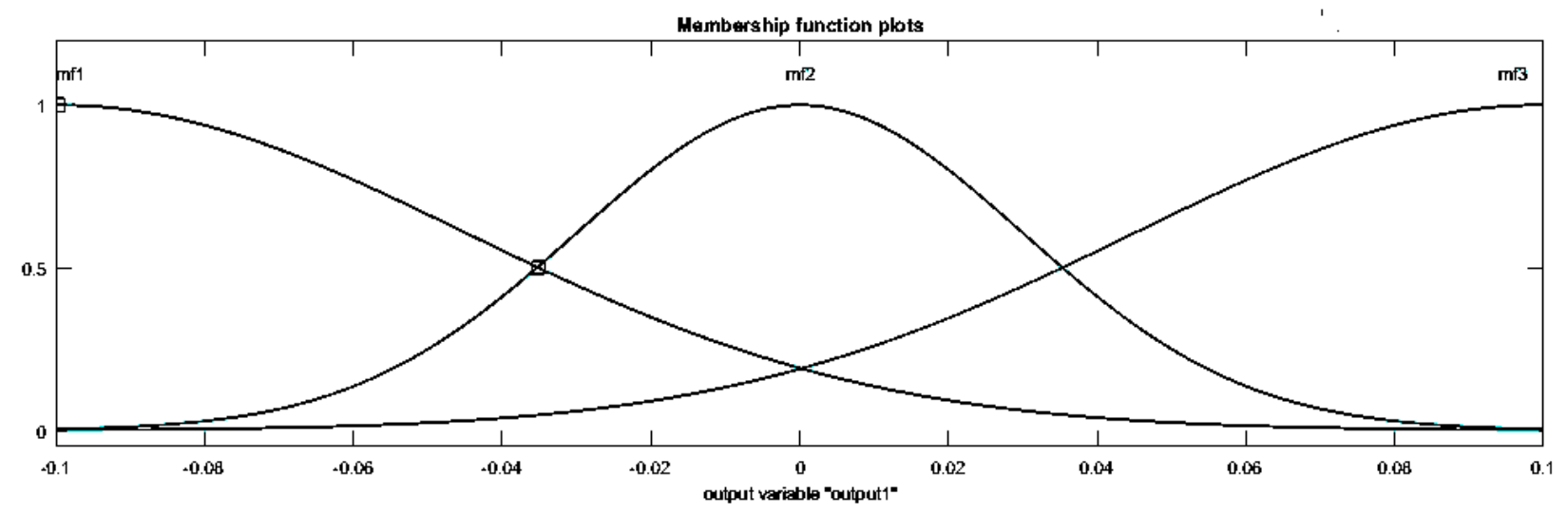

Figure 4.29 Demonstration - Gaussian output membership functions

Figure 4.30 shows the output results using triangular IMFs and OMFs. Through the transition of the MFs, it can be seen that the middle section of input yields almost a constant value of output due to the plateau through a fairly large range of values. This property is not desirable at all since if input values falls within this range, it means that the controller basically would have no change in output value, or in other words, the controller is not reacting to the change of input at all. 


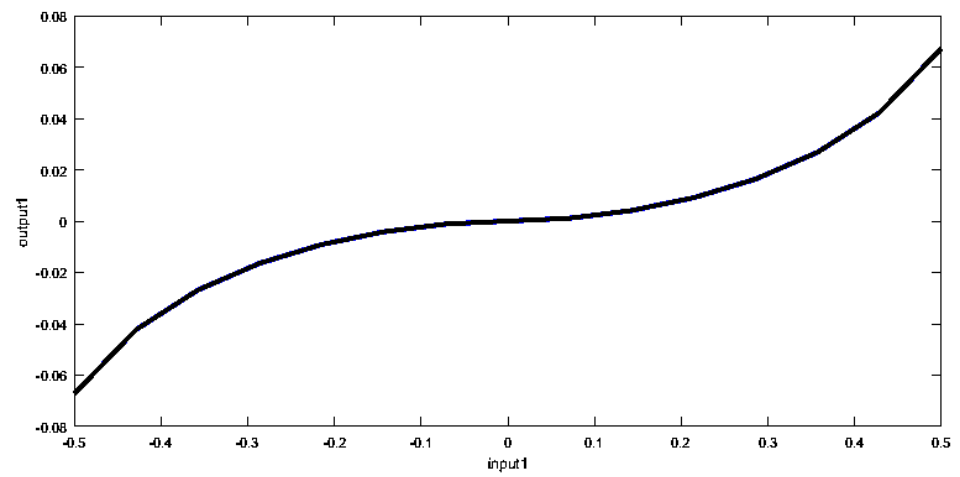

Figure 4.30 Demonstration - output results using triangular input and output membership functions

On the other hand, outside the plateau portion, the transition of smaller output values to the larger output values is too slow, which will cause the controller not responsive enough for the inputs. These two properties explain the offset problem in Figure 4.24, as well as the inability to maintain $\mathrm{DGI}_{\mathrm{m}}$ at the set-point.

Figure 4.31 shows the output results using Gaussian IMFs and OMFs for the fuzzy PD controller. Using this shape, the middle section of the output curve is close to a linear increase, which means that the controller will be responsive towards error that slightly deviates from 0 and able to maintain $\mathrm{DGI}_{\mathrm{m}}$ at the set-point with no offset. The rest of the curve demonstrates a much more sensitive response of controller to error or rate of change with large output values between the range of $[-0.4,-0.1]$ and $[0.1,0.4]$ when compared to Figure 4.30 .

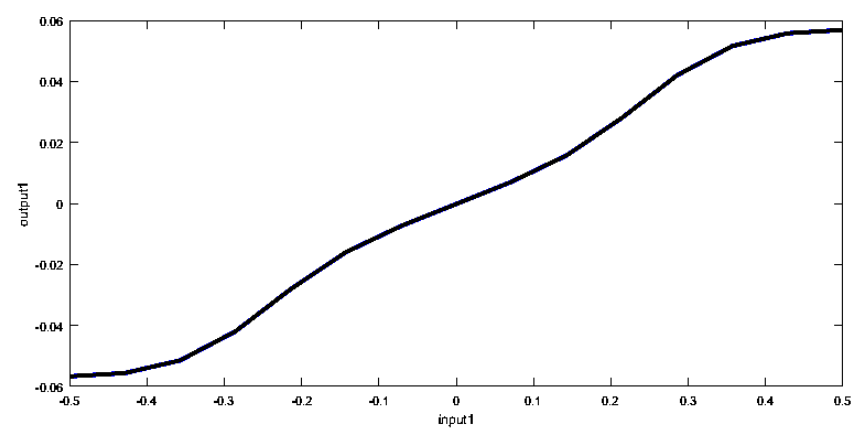

Figure 4.31 Demonstration - output results using Gaussian input and output membership functions 
For the overall shape, as mentioned earlier, the more linear the output curve, the better the performance when dealing with error. However, it is very difficult to manually design a controller with strictly linear output. If not possible, then the overall curvature demonstrated in Figure 4.31 should yield reasonably well controller performance.

With the completed discussion of curve shapes, the reason behind the design of shape of output curves of PD controller can be understood better. Figure 4.32 shows the output curve of the PD controller for the entire range of error values. The curve was designed as linear as possible especially within the range of $[-1,1]$. Figure 4.33 shows the output curve for various rate of change values. For this parameter, it is intentionally designed (by adding the extra two membership functions) such that the slope at $[-0.1,0.1]$ is smaller. This is done so to ensure the controller less sensitive to the rate of change when $\mathrm{DGI}_{\mathrm{m}}$ is settled at the set-point.

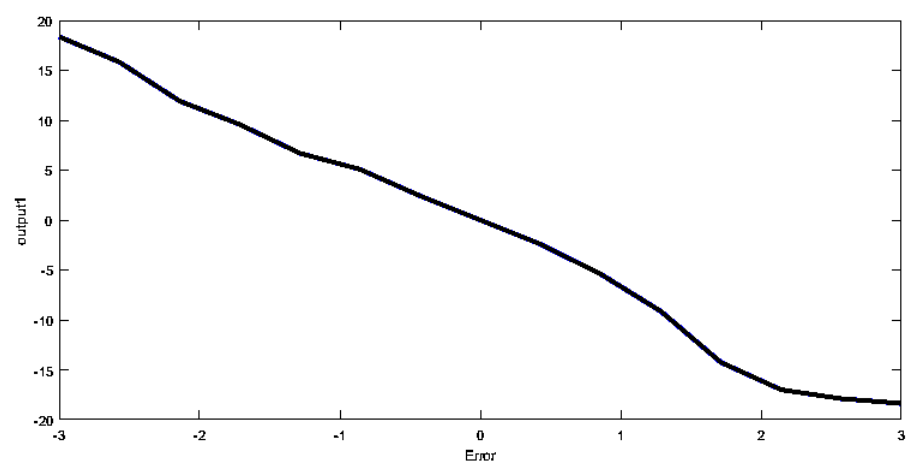

Figure 4.32 Designed fuzzy PD controller output curve - output vs error

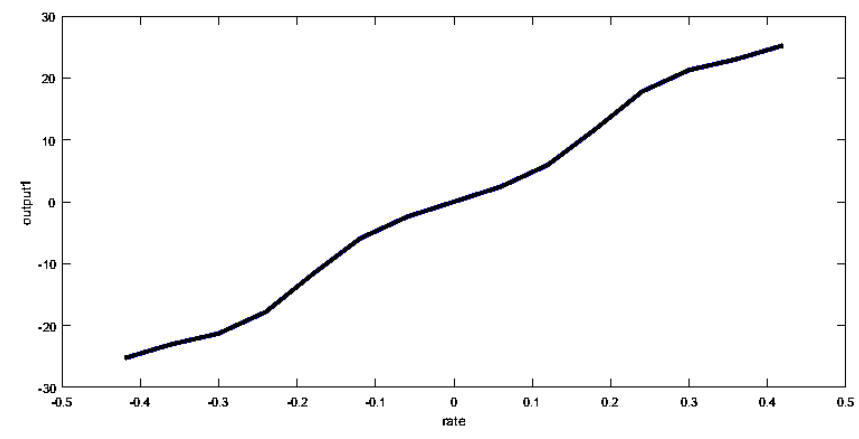

Figure 4.33 Designed fuzzy PD controller output curve - output vs rate of change 


\subsection{Chapter Summary}

This chapter discussed about the development of fuzzy logic controller designed to control the electrochromic window. Through trial and error and by studying the control surface diagram that fuzzy logic generates, a final version was developed deemed applicable for this project. The final control performs with no overshoot and a settling time within 90 s from clear to dark and within 200s from dark to clear. Although there were small fluctuations when $\mathrm{DGI}_{\mathrm{m}}$ is supposed to settle at the set-point, the amplitudes were small enough to be neglected. Seven Gaussian membership functions for both inputs (error and rate of change of error) and nine for the output (change in voltage) with corresponding forty-nine fuzzy rules were created to achieve the desired performance. 


\section{Chapter 5 Control System Implementation}

Chapter 3 presented the environmental and equipment setup. There are still more implementation issues (hardware and software) that need to be discussed in order to complete the entire system.

\subsection{Simulink Model}

Figure 5.1 shows the connection of all the devices that were used to control the electrochromic window. The system consisted of an $8 \mathrm{~V}$ and $10 \mathrm{~V}$ power supply, a $4-20 \mathrm{~mA}$ data acquisition board with RS232 interface that converted light sensor current into digital signal which in turn can be sent to the computer through a RS232 to USB converter (Figure 5.2).

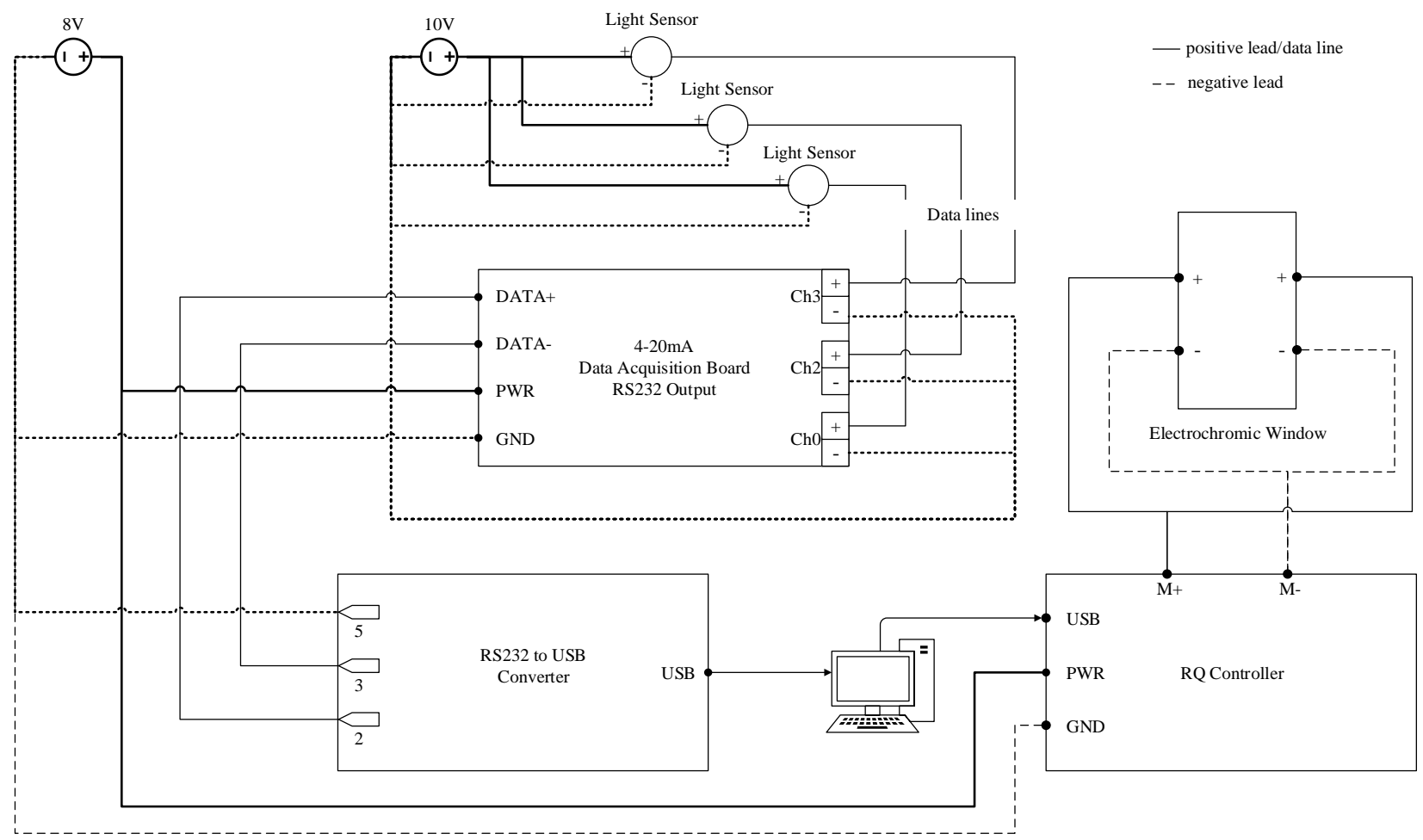

Figure 5.1 Hardware connection wiring diagram 


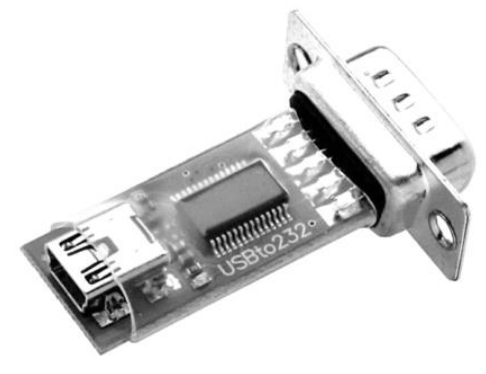

Figure 5.2 RS232 to USB converter

After light sensor signal was collected, the DGI value could be calculated. The calculation was done in the background using Matlab graphical user interface GUI that was linked to Simulink model which contained the fuzzy logic controller (Figure 5.3). The output from the fuzzy controller $\Delta \mathrm{V}$ was then passed back to the GUI, which kept track of the voltage $\mathrm{V}$ applied to the RQ controller, to output a final voltage value for the RQ controller for adjusting the window transparency. It is worth to notice that each positive output $(\mathrm{M}+)$ from RQ controller was connected the two positive leads at the EC window, same for negative leads which were connected to M-.

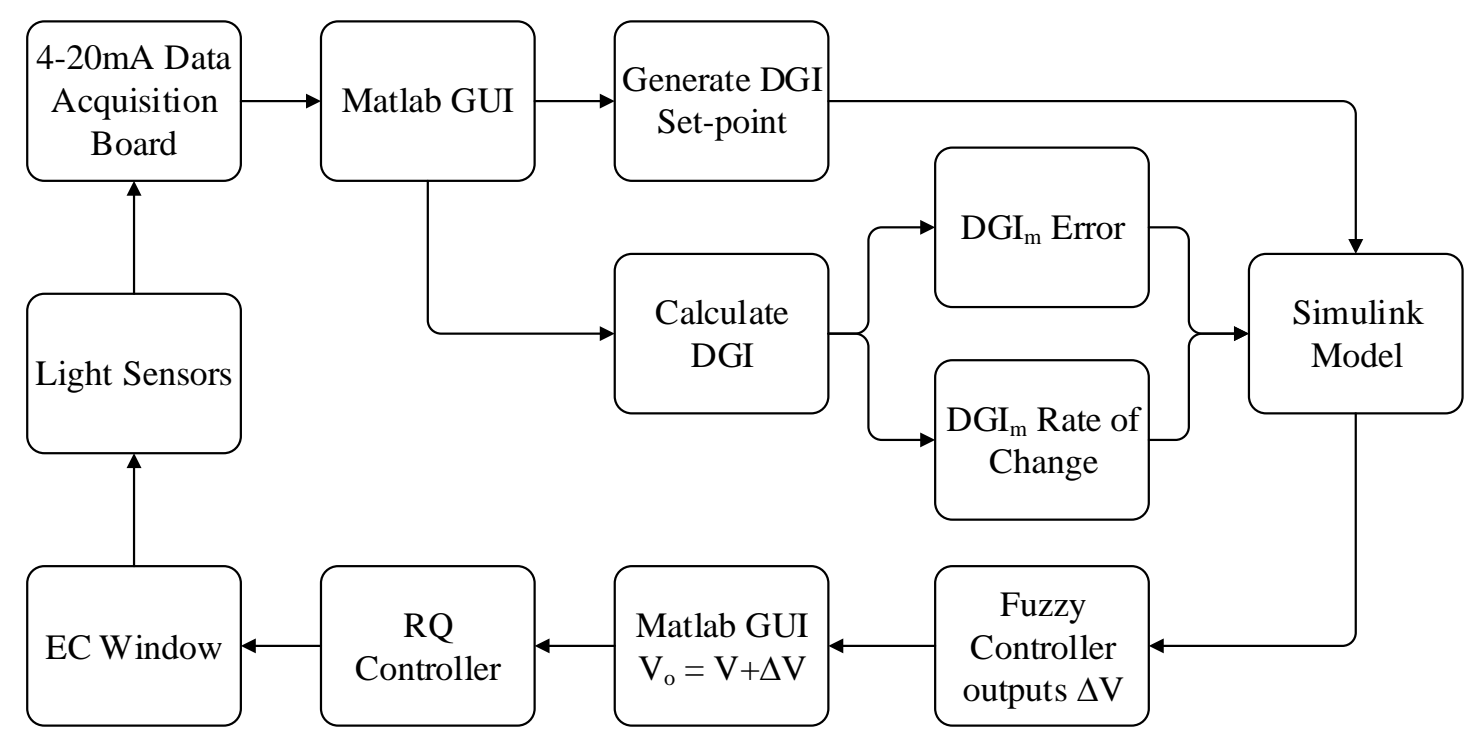

Figure 5.3 Complete flow chart of system 
Since Simulink could not connect to the RQ controller, but Matlab was able to by using the serial communication commands, therefore Matlab GUI was used as an interface to send to output command $\Delta \mathrm{V}$ from the fuzzy logic controller towards the RQ controller.

During the experiment, it was noticed that the controller had made many very small adjustments that were practically meaningless when maintaining a stable tint at the set point, therefore in the GUI code, the values of the fuzzy logic controller output within the range of $[-2$, 2] were neglected.

Figure 5.4 shows the Simulink model that mainly contained the fuzzy logic controller. The GUI passed both the DGI $\mathrm{m}$ and rate of change of error to the "DGI $\mathrm{m}_{\mathrm{m}}$ " block and the ROC block respectively. The desired glare level that was defined by the user, was passed from the GUI to the "DGISP" block. Both constraint blocks at the input of fuzzy controller ensured that the range of values for the controller was within the range that was designed in chapter 4 . The "Window Tint Limit" rather than working as a real constraint, it acted as the block that passed the output value back to the GUI since the controller would not output a value that is beyond expectation.

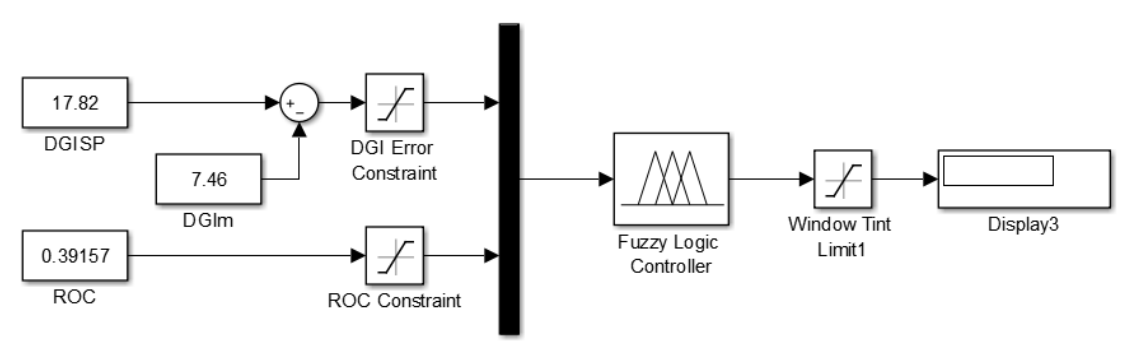

Figure 5.4 Software implementation - Simulink model

The GUI as shown in Figure 5.5 is separated into left and right two parts. The left part is the external light source control panel to control the light bulbs wirelessly, and right below it is the $\mathrm{DGI}_{\mathrm{m}}$ graph that updated continuously throughout the entire experiment, this section was 
mainly used by the author to control and observe the environment and had no significant use for the user. The right part is the user panel, where the user could control the window tint by adjusting the desired DGI level. This included adjusting the window tint by pressing the " $+3 /-$ 3/+2/-2" buttons when "Auto Detect" was enabled. If "Manual Control" was enabled, the same buttons would adjust the window tint by directly controlling the RQ voltage output. In addition, the user could choose to turn the window fully clear or fully dark.

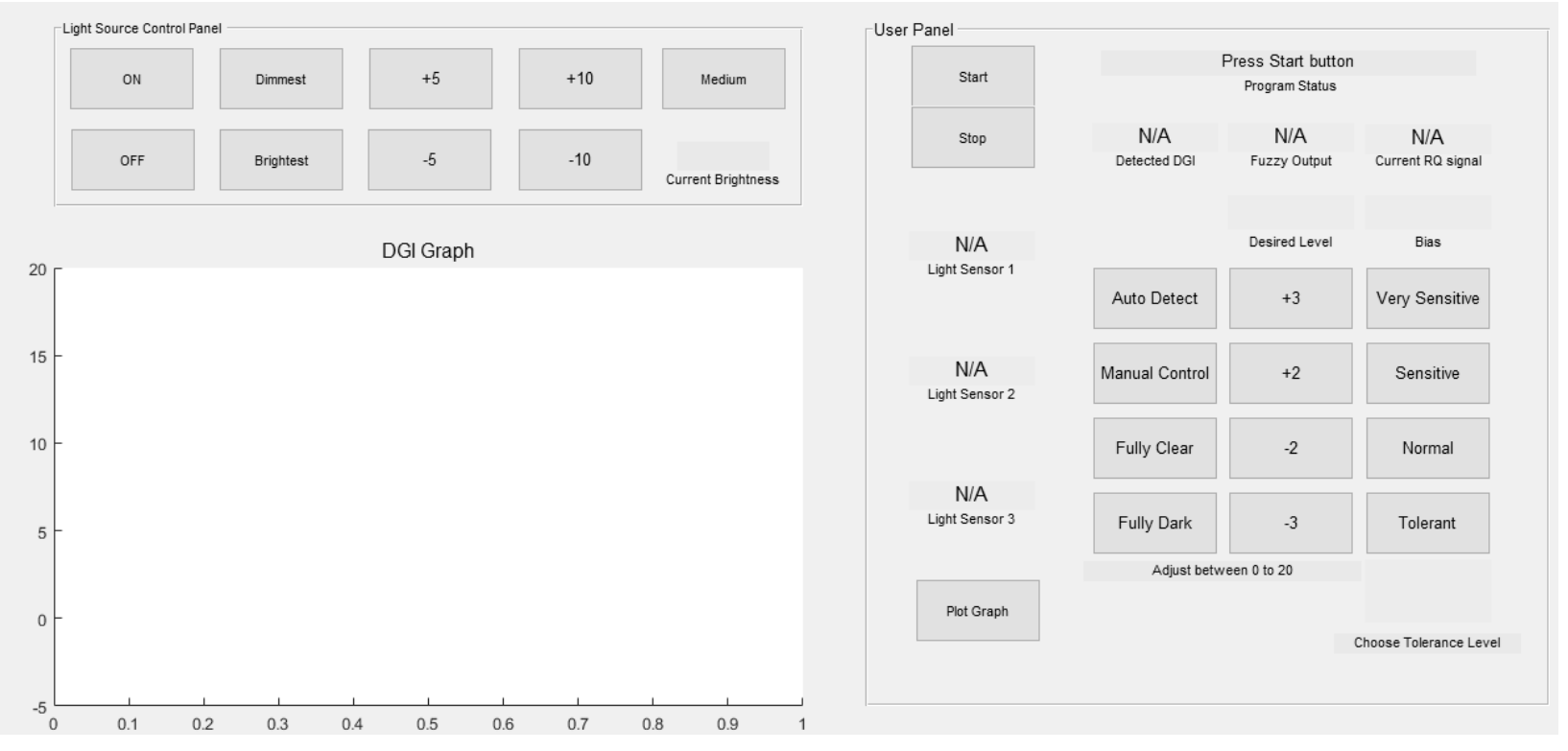

Figure 5.5 Graphical User Interface

Additionally, the user could choose the glare tolerance level on the right-most column based on one's own preference. What would do is to add a constant bias towards the DGISP value (Figure 5.6). This way, when the user chose the setting, the window would be constantly clearer or darker than a normal setting. For example, with a normal setting, if the window got completely dark when DGI was 0, with "Very Sensitive" setting, the window would get completely dark when DGI was 4 . The advantage of doing such is to ease out the operation needed for the user to drastically change the window tint setting. 


$$
D G I_{o}=D G \mathrm{I}_{\mathrm{SP}}+\text { bias }
$$

Where $D G I_{o}$ is the set point for the fuzzy logic controller and $D G I_{S P}$ is the intermediate set point before adding the user bias. If there is no user bias, then $D G I_{o}$ and $D G I_{S P}$ are the same.

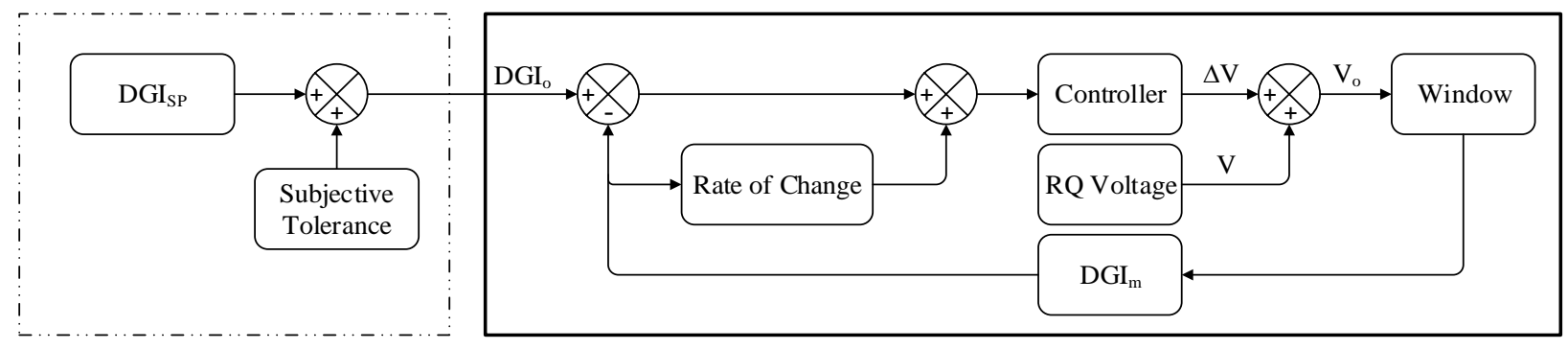

Figure 5.6 Electrochromic window control integrated with user tolerance level

Table 5.1 shows the suggested bias added towards the DGI. It was mentioned in previous chapters that tint changes within 1 unit of DGI is not noticeable, therefore the minimum increment/decrement is 2 units, while 3 units offers more distinct contrasts between normal setting and other settings. In Figure 5.6 the portion with dashed line box represents equation 7 , while the solid line box contains the control system that was discussed in chapter 4 .

Table 5.1 Suggested DGI bias values at various glare tolerance levels

\begin{tabular}{|c|c|}
\hline Tolerance level & bias \\
\hline Very Sensitive & -5 \\
\hline Sensitive & -3 \\
\hline Normal & 0 \\
\hline Tolerant & -3 \\
\hline
\end{tabular}

McCluney [18] suggested that when considering suitable lighting conditions for occupants, the age factor plays an important role generally for specific age groups. Over the 
years, experience showed that the majority of occupants will find the following standard to be perceived as comfortable lighting.

Firstly, Table 5.4 shows the recommend illuminance values for various task categories. Group A to C are tasks that require low visual acuity, while Group D to I require higher to highest acuity that human will ever perform. Due to age considerations, there are ranges of values recommended. For different ages, the weighing factor $(-1,0,+1)$ is being used. For example, for a painting artist, the environment that he requires falls into category F. Considering that he is already 60 years old, according to Table 5.3, a weighing factor of $(+1)$ is required. Let's say the room reflectance is $50 \%$, then a weighing factor of $(0)$ is chosen. Lastly, since accuracy of his work is considered as important, a weighing factor of (0) is chosen. The cumulative weighing factor is $1+0+0=1$, therefore the average illuminance is suitable for him. According to this method, the values of weighing factor for categories D to I can only be between -3 and +3 . If the factor is -3 or -2 , then pick the minimum illuminance value; if the factor is +2 or +3 , then pick the maximum value, otherwise pick the average illuminance. The similar concept applies for categories A to C.

Table 5.2 Weighing factors for categories A to $C$

\begin{tabular}{|l|c|c|c|}
\hline \multirow{2}{*}{} & \multicolumn{3}{|c|}{ Weighing Factor } \\
\cline { 2 - 4 } & -1 & 0 & +1 \\
\hline Occupant's Age & $<40$ & $40-55$ & $>55$ \\
\hline Room surface reflectance & $>70 \%$ & $30 \%-70 \%$ & $<30 \%$ \\
\hline
\end{tabular}


Table 5.3 Weighing factors for categories D to I

\begin{tabular}{|l|c|c|c|}
\hline \multirow{2}{*}{} & \multicolumn{3}{|c|}{ Weighing Factor } \\
\cline { 2 - 4 } & -1 & 0 & +1 \\
\hline Occupant's Age & $<40$ & $40-55$ & $>55$ \\
\hline Room surface reflectance & $>70 \%$ & $30 \%-70 \%$ & $<30 \%$ \\
\hline Speed and/or accuracy & Not important & Important & Critical \\
\hline
\end{tabular}

Table 5.4 Recommended illuminance for various tasks

\begin{tabular}{|l|c|c|c|c|}
\hline \multicolumn{2}{|c|}{} & \multicolumn{3}{|c|}{ Illuminance (lux) } \\
\hline Activity & Category & Min & Ave & Max \\
\hline Public spaces with dark surroundings & A & 20 & 30 & 50 \\
\hline Simple orientation for short temporary visits & B & 50 & 75 & 100 \\
\hline $\begin{array}{l}\text { Working spaces where visual tasks are only occasionally } \\
\text { performed }\end{array}$ & C & 100 & 150 & 200 \\
\hline $\begin{array}{l}\text { Performance of visual tasks of high contrast or large size } \\
\text { Performance of visual tasks of medium contrast of small } \\
\text { size }\end{array}$ & D & 200 & 300 & 500 \\
\hline $\begin{array}{l}\text { Performance of visual tasks of low contrast or very small } \\
\text { size }\end{array}$ & F & 1000 & 1500 & 2000 \\
\hline $\begin{array}{l}\text { Performance of visual task of low contrast and very small } \\
\text { size over prolonged period }\end{array}$ & G & 2000 & 3000 & 5000 \\
\hline Performance of very prolonged and exact visual tasks & H & 5000 & 7500 & 10000 \\
\hline $\begin{array}{l}\text { Performance of very special visual tasks of extremely low } \\
\text { contrast and small size }\end{array}$ & I & 10000 & 15000 & 20000 \\
\hline
\end{tabular}

The method that McCluney [18] suggested dealt with the suitable illuminance condition for various tasks and age group, since the categorization were based on illuminance conditions while the purpose of this project is to deal with glare level, the results cannot be used directly. However, this does provide an insight of how to further improve the automation concept that is being investigated in the project to determine an even more meaningful DGI value to each individual. 


\subsection{Personalized DGI Set Point}

The previous section mentioned two main ideas of categorization: 1) user tolerance level, 2) categorization based on the user's age and activity at the space occupying. Both can actually be integrated together to determine the $\mathrm{DGI}_{0}$. This proposal can supplement the lacking categorization element in the control system presented in chapter 4 , but deems to be the basis for dealing with human perception with fuzzy logic for this thesis.

There are many different factors that can affect a person's perception of glare. For this project, three of the most direct factors are taken into account - age, eye condition, and activity that the user is performing. However, to have very accurate data, it requires a lot of data for statistical analysis which is not available. Therefore, some theoretical scenarios are done as demonstration of the concept.

The idea of the method is to generate a DGIsP from fuzzy logic that can incorporate as many considerations as possible. However, not all conditions can be related directly. Age and activity that the user is performing certainly can be directly related in a way that, the more demanding the work a person is performing, the more lighting one requires. Therefore, the amount of glare that the person feels acceptable would generally be higher. The level of glare is also related to age. The younger the user is, the more sensitive he/she is towards it. Therefore, these two considerations can be put together.

On the other hand, considering a person's eye condition, the logic towards getting a glare level is "compared to a normal person, how much more glare does he/she desire/accept". Instead of considering the glare level directly, what more suitable is to base on the original determination of glare level, adjust it by increasing or decreasing to a certain extent. There are many considerations that are within this category. For example, people with light eye color are more 
sensitive to light than people with darker eye color. Other conditions such as corneal abrasion, various types of uveitis, albinism, iritis, keratitis can all affect the acceptable level of glare compared to normal people from the same age group. The more extensive the research about various eye conditions on the acceptable level of glare, the better the result will be.

Figure 5.7 shows the complete diagram of all the window controlling methods that a user could choose from based on all the discussions in this chapter. As a recap, the user can choose completely automatic mode, which allows the fuzzy logic to determine the suitable DGIsp based on the initial user input (or obtained from database), including age and his/her eye condition, while the user can always select the current activity that he/she is performing to further adjust the fuzzy output. On the other hand, there are two manual modes that are available to choose. The semi-automatic mode allows the user to select certain DGIsP level, and the PD controller would adjust the window tint to maintain the glare level whenever possible. This is called semiautomatic since this requires direct input from user, and the window tint changes according to environment. Lastly, the fully manual mode is a direct user controlling method to adjust window tint by adjusting the voltage applied to the window without any influence from light sensing equipment or fuzzy logic.

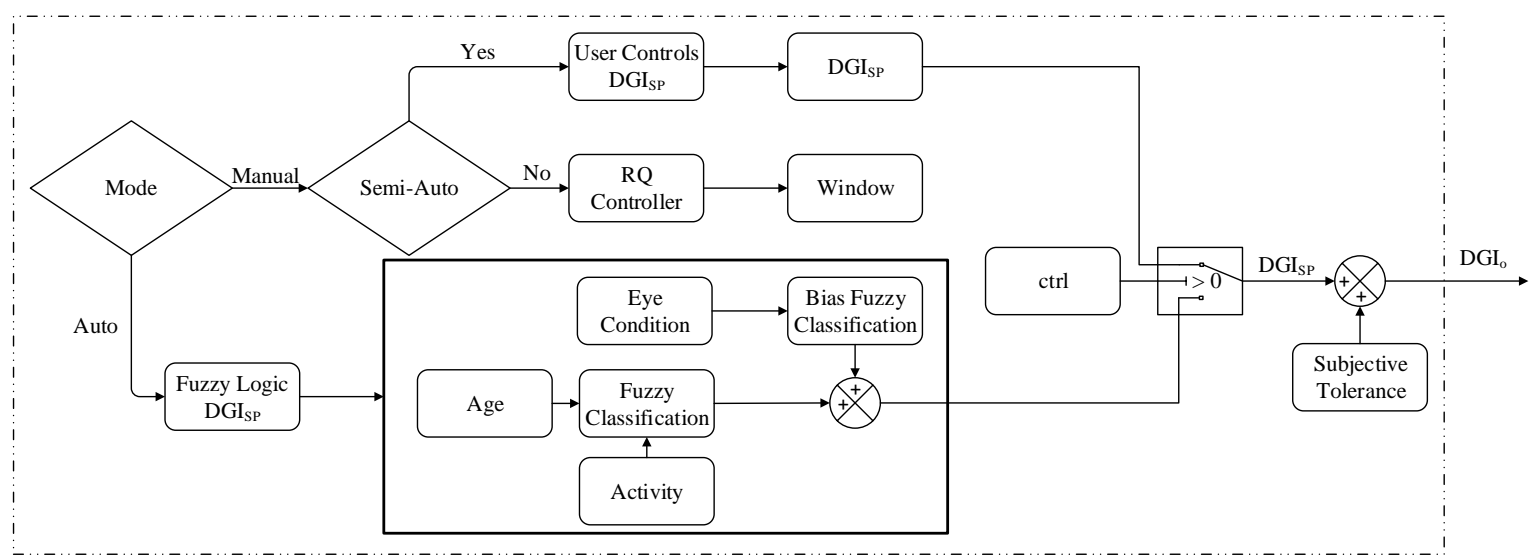

Figure 5.7 Full electrochromic window control integrated with influence of human perception 
Since there is no large amount of statistical data from users to work with, Table 5.5 suggests some preliminary DGISP values that were suggested by a few participants, and random normally distributed data is generated by treating the values as the respective means. An assumption is made that the older the user is, the more lighting he/she requires for various activities. For activities that require more concentration, then more light is required, thus acceptable glare level will also be higher. Another procedure was made with regards to the artificial lighting. For different categories of activities that were being performed, participants were asked to use a mobile application to control the illuminance level inside the mock up. If desired, they can change the color temperature to the better suit their feelings.

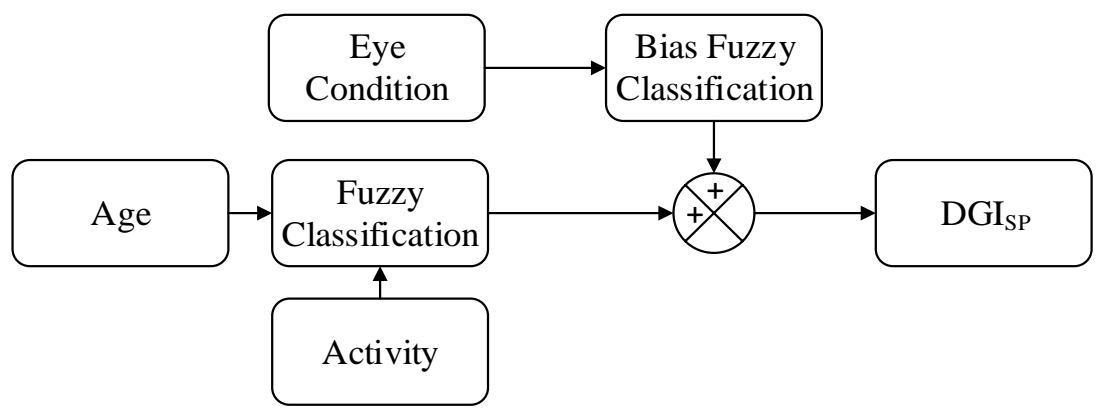

Figure 5.8 Fuzzy Logic for DGISP

Table 5.5 DGISP for various activities performed by different age groups

\begin{tabular}{|c|l|c|c|c|c|}
\hline \multicolumn{2}{|c|}{} & \multicolumn{4}{|c|}{ Activity } \\
\cline { 3 - 6 } Age & Movie/Sleep & Eat & Read & Work \\
\hline \multirow{3}{*}{ Age } & Kid & 0 & 3 & 5 & 10 \\
& Young & 0 & 3 & 8 & 11 \\
& Middle Age & 0 & 4 & 10 & 13 \\
& Old & 0 & 8 & 13 & 15 \\
\hline
\end{tabular}

McCluney [18] suggested that the age of eye boundaries between young and middle age is 40 years old while that of middle age and old is 55 years old. These boundaries are 
incorporated into the design of fuzzy, while all other age boundaries are completely hypothetical. As for "Activities", since it is always required to have an input value for the fuzzy logic to perform calculation, it is required to have a numerical way to distinguish what the user is doing. It can be done in two ways:

1) By using illuminance values based on Table 2.3 in chapter 2, which suggested some mean illuminance values at various environments that is intuitively representing various activities. For instance, the dining room illuminance is about 200 lux, library is 350 lux, office is 500 lux. Though there is no value for a theatre, any low lux values will be treated as the user is watching movie.

2) There can be a record of lighting preference for respective activities, and the average of each preference can be used as the input for the fuzzy logic. This method is desirable when indoor artificial lighting is taken into consideration, and the design of the membership functions for "Activities" can be more tailored towards each user.

The first method is simple and the designer of the system can virtually categorize various activities by inputting any desired value that represent each activity. The values thus do not have to meaningful. The second method requires more work for designer by adjusting the fuzzy logic for each user, but the benefit is that it can fuse with environment by reacting in a way that the more illuminance is required, the clearer the window will be, thus letting in more light and reducing stress on artificial lighting. Since the benefit of the second method overrules the cons, the following discussion will be the design of fuzzy logic system to satisfy the various DGIsP selections based on the concept of the second method. 
Figure 5.9 and Figure 5.10 show the preliminary input membership functions that approximate the range of values for all activities and age groups mentioned earlier. In terms of the output, the theoretically recorded data from users are used as the basis to output a DGISP value.

With some small samples of data, it might be easy to design an FIS that can generate the desired output values, but with multiple categories of data from multiple outputs, the design of an FIS manually using Mamdani model as in chapter 4 would be very difficult.

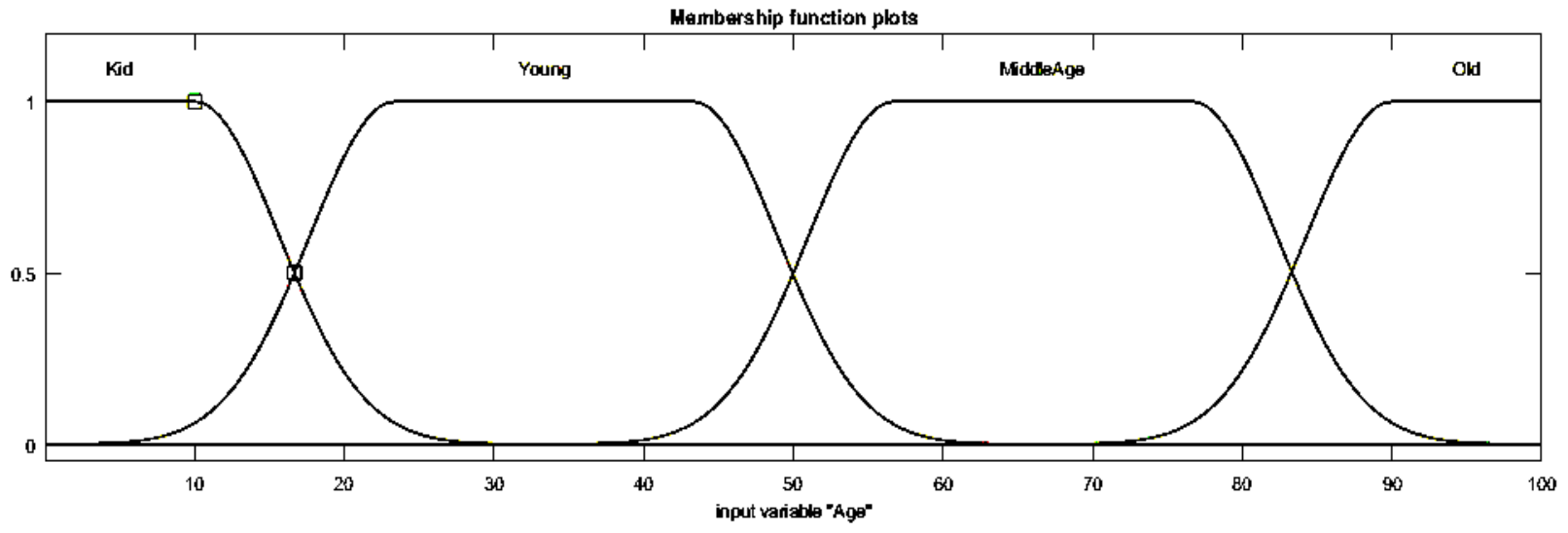

Figure 5.9 Input membership functions for "Age"

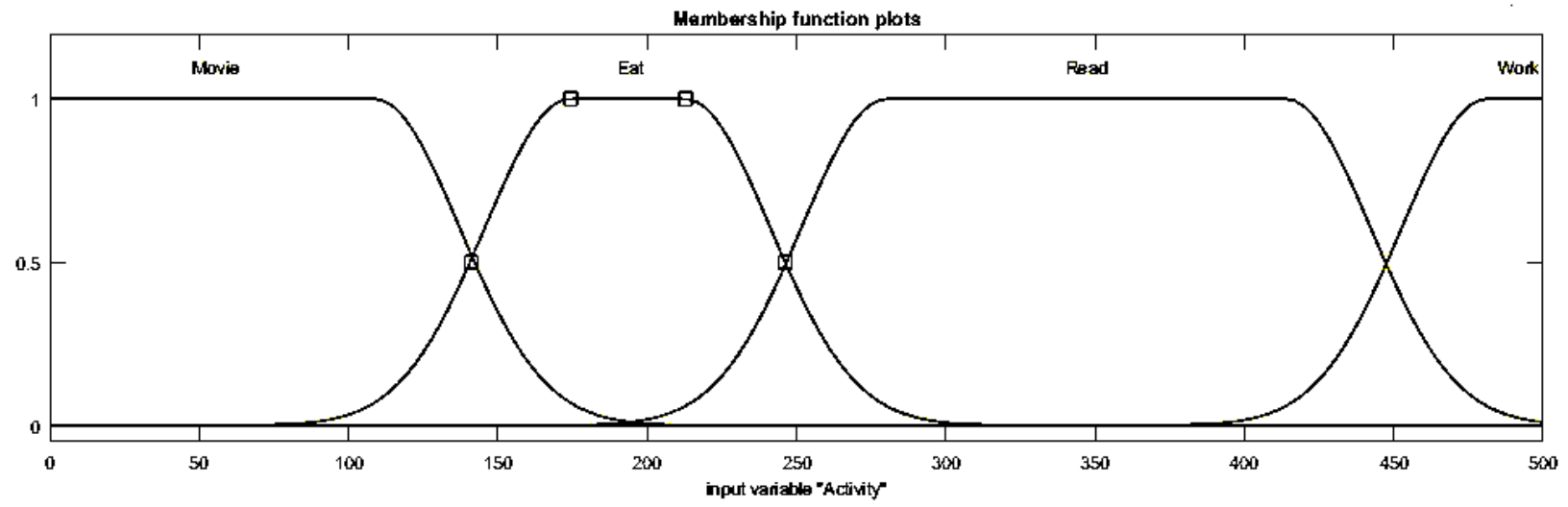

Figure 5.10 Input membership functions for "Activity" 
In view of this, Adaptive Neural-Fuzzy Inference System ANFIS can be of a great tool to adjust the output membership functions to generate proper output values through neural network (Figure 5.11) by forward pass and backward propagation. In order to use ANFIS, the FIS model must be a Sugeno model. The design of membership functions was the same as a Mamdani model, it was the output membership functions that were different.

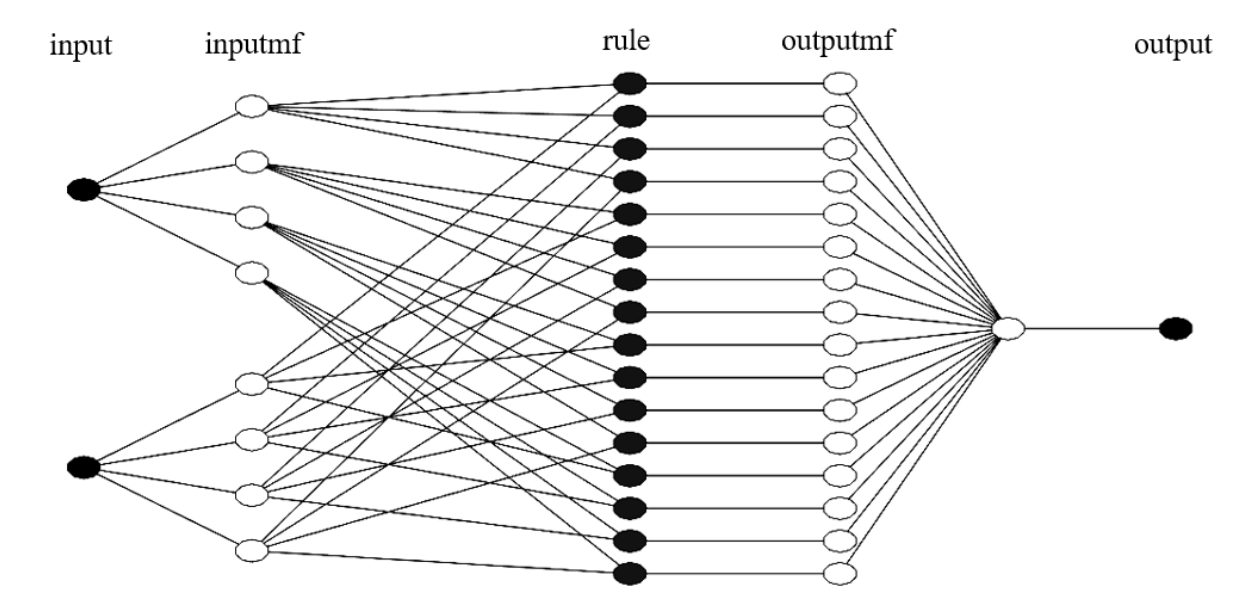

Figure 5.11 Neural Network for FIS training

As shown in Table 5.5, this demonstration consists of 4 IMFs and OMFs and accordingly, 16 rules are required as shown in the hidden layer of the neural network. Suppose that data is available for all activities and all age groups. Before putting the data into ANFIS for training, the moving average of the data is obtained. Otherwise it is difficult to generate a FIS that is capable of producing continuous and consistent results within each specific category.

When the preliminary input membership functions and the data were put into ANFIS for training, it was realized that regardless of effort, no acceptable control surface could be obtained that could allow the FIS to generate output that was close to the data in Figure 5.12. The reason was because of improper design of IMFs in the first place that could make it impossible for ANFIS to calculate the suitable OMFs to match the data. 


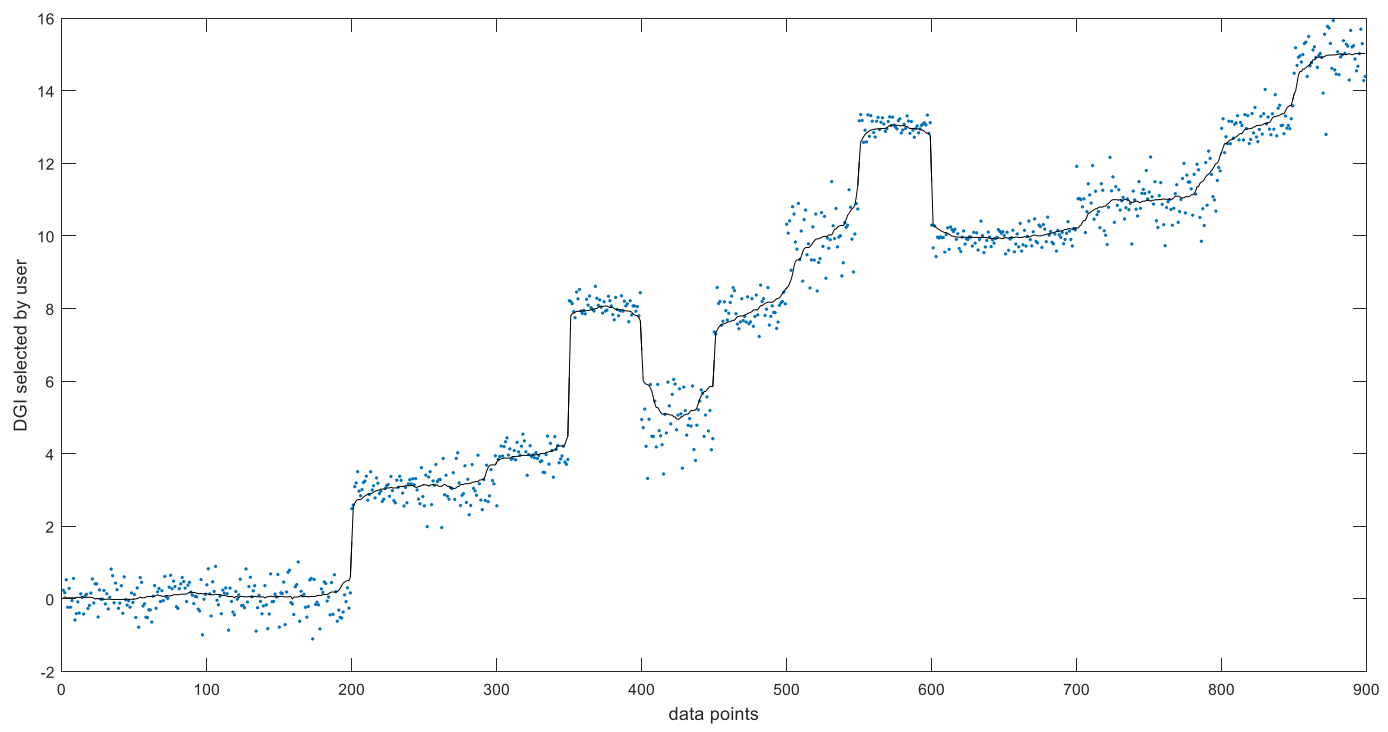

Figure 5.12 Moving average of various DGI $I_{S P}$ level selected by users

The problem with the improper control surface as shown on the left side in Figure 5.13 is that it completely lacked the distinct categorization of the ages and activities that the FIS was meant to be designed for. The transition from one to another did not match with the pattern as that in the training data, and the values that it generated were quite different from what was wanted.
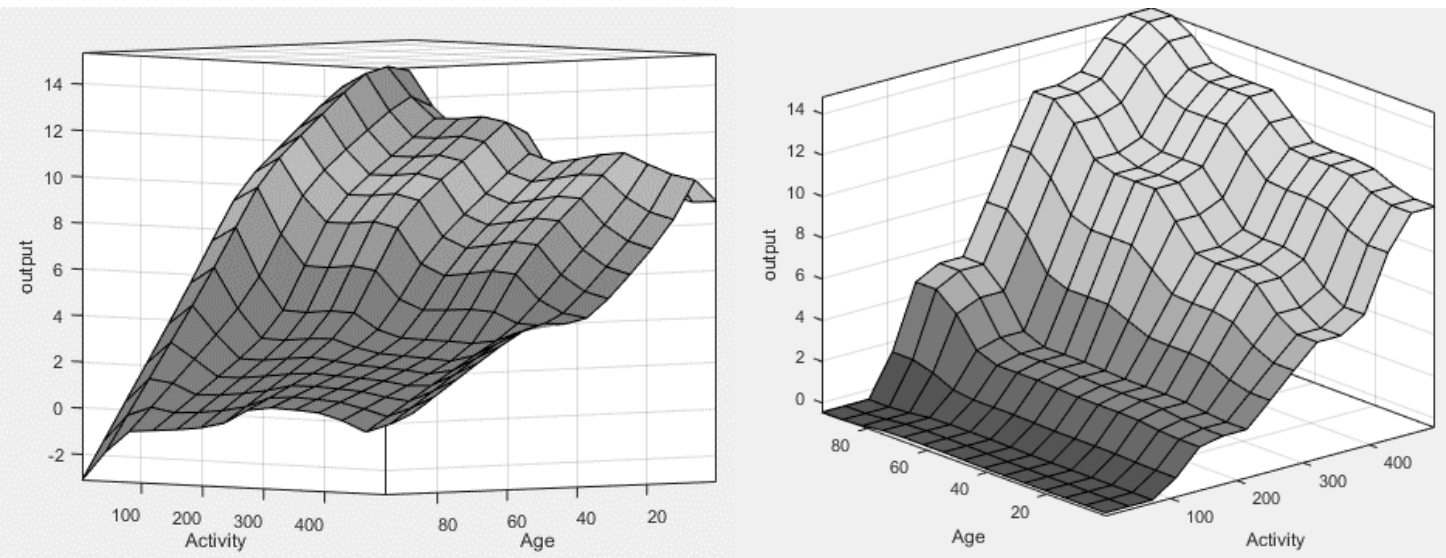

Figure 5.13 Improper control surface (left) vs proper control surface (right) 
In order to obtain a proper FIS, instead of designing the IMFs manually, the grid partitioning option was chosen, and 4 IMFs (Figure 5.14), 4 OMFs (Figure 5.15) were specified. This option allowed ANFIS to generate the entire FIS system on its own based on the data that were given. Initially, Gaussian MFs were chosen, however, it was found that trapezoidal MFs were even better.

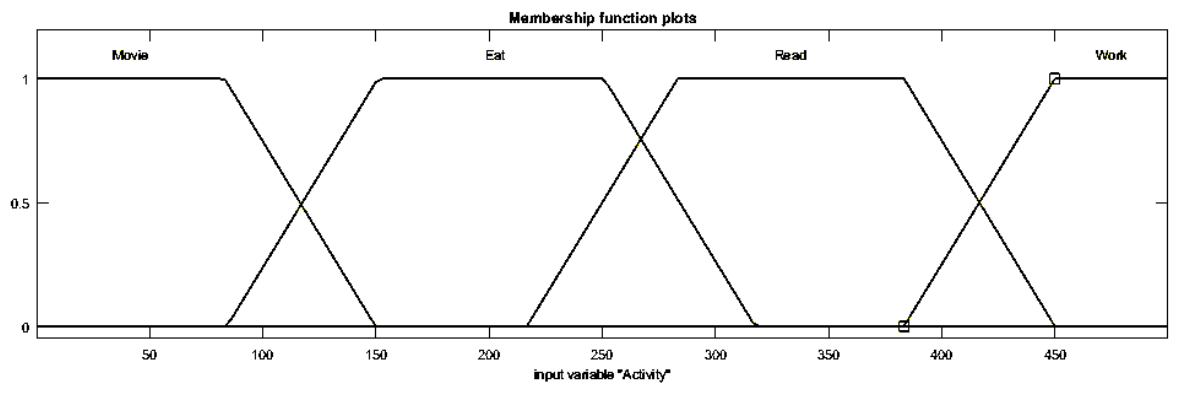

Figure 5.14 Input membership functions for "Activity" generated by ANFIS

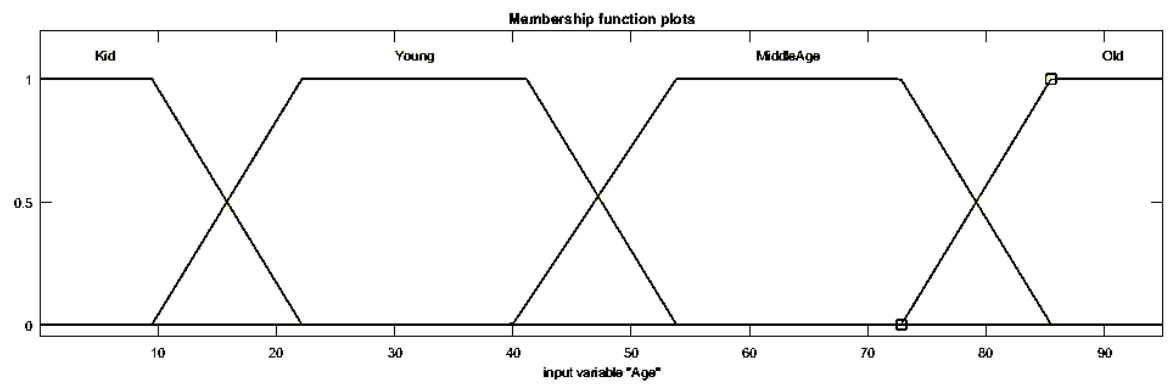

Figure 5.15 Input membership functions for "Age" generated by ANFIS

The control surface as seen on the right side in Figure 5.13 is the control surface of the FIS generated by ANFIS. For better viewing of results, 9 points with each of 50 lux apart were chosen for each age group and is shown in Figure 5.16. The overall trend of the result matched quite closely with what was desired, and showed that for each activity the higher the desired illuminance and the older the user, the higher the DGI output. 
Nonetheless, there were still some minor changes that should be made in order to satisfy the pre-defined numbers in Table 5.5. The first thing that should be adjusted was that, with the range between 50 lux to 100 lux, the DGI output should be 0 . Second, instead of having an almost same range of DGI output in the range of 300 lux to 400 lux, it was more desired to have an increasing magnitude of DGI to match the idea that the window should turn clearer as the required illuminance increases. This is more of an important issue while the user is working or reading, but when the user is eating, it might not be much of an issue.

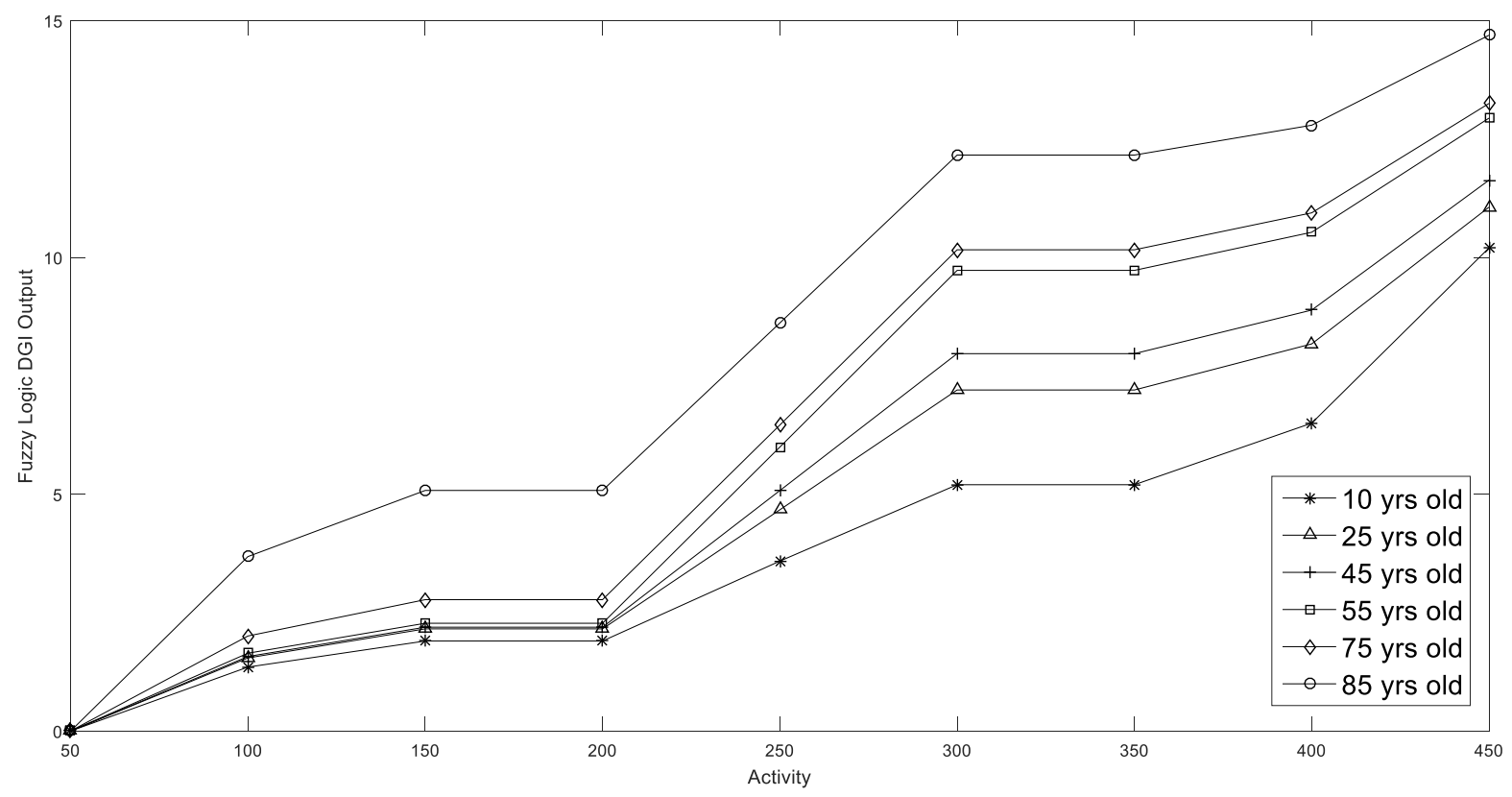

Figure 5.16 Result from fuzzy logic DGI output for various ages after ANFIS training

Figure 5.17 to Figure 5.20 and Table 5.6 show the complete FIS that was adjusted from the ANFIS results. The changes that were considered required were satisfied and the output values matched very closely with that in Table 5.5. 


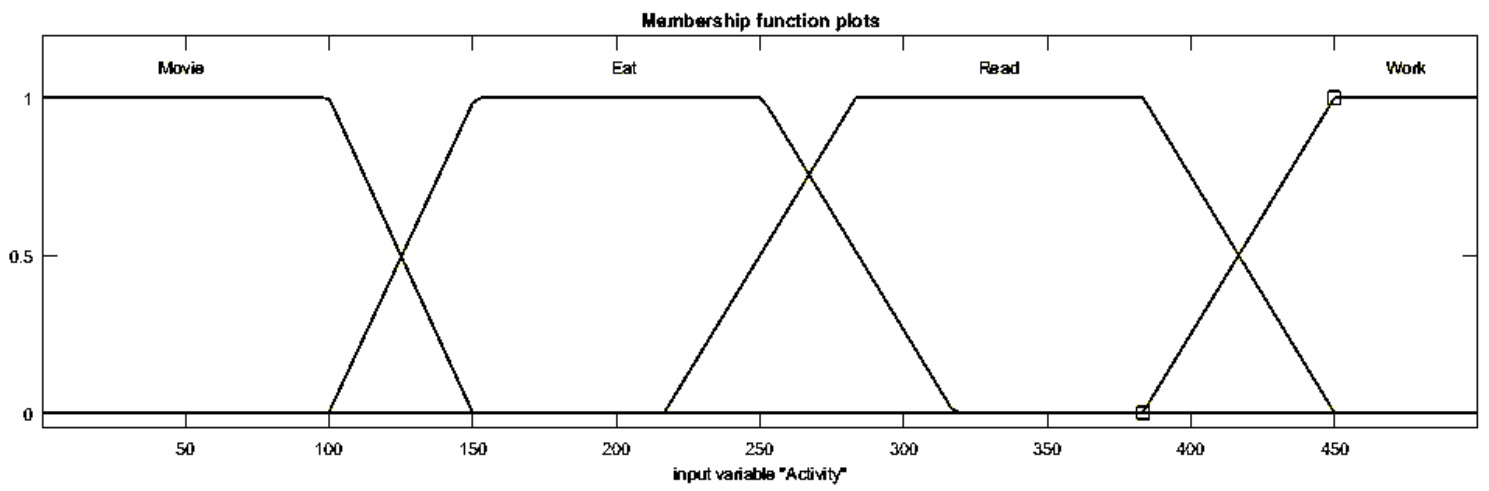

Figure 5.17 Input membership functions for “Activity” after adjustment

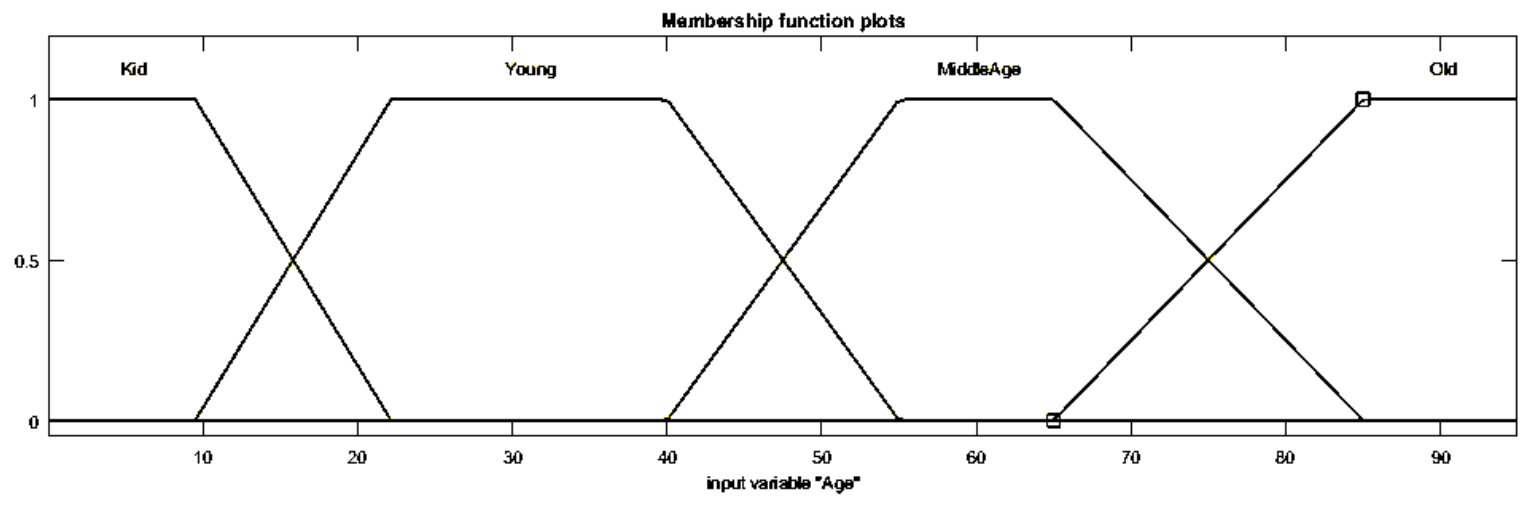

Figure 5.18 Input membership functions for "Age" after adjustment

Table 5.6 Output membership functions for the fuzzy inference system computed by ANFIS

\begin{tabular}{|c|c|c|c|}
\hline MF no. & Constant value & MF no. & Constant value \\
\hline 1 & -0.2791 & 9 & 5.193 \\
2 & -0.2774 & 10 & 7.205 \\
3 & -0.2779 & 11 & 9.725 \\
4 & -0.463 & 12 & 12.27 \\
5 & 1.896 & 13 & 10.2 \\
6 & 2.157 & 14 & 11.06 \\
7 & 2.275 & 15 & 12.94 \\
8 & 5.203 & 16 & 14.77 \\
\hline
\end{tabular}




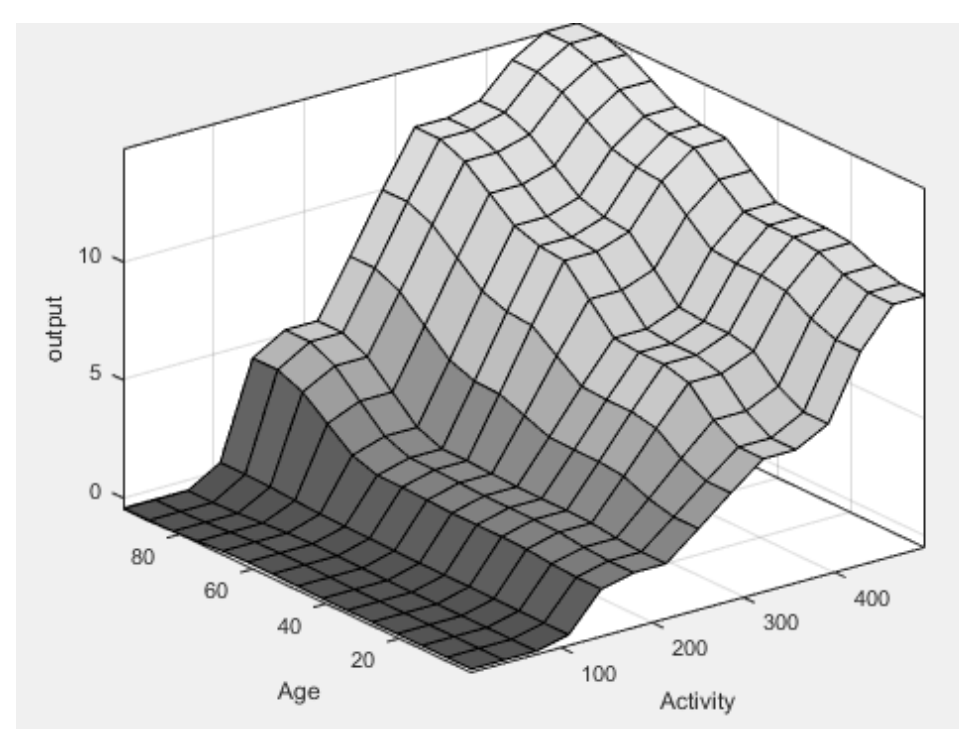

Figure 5.19 Control surface diagram of fuzzy inference system after adjustment

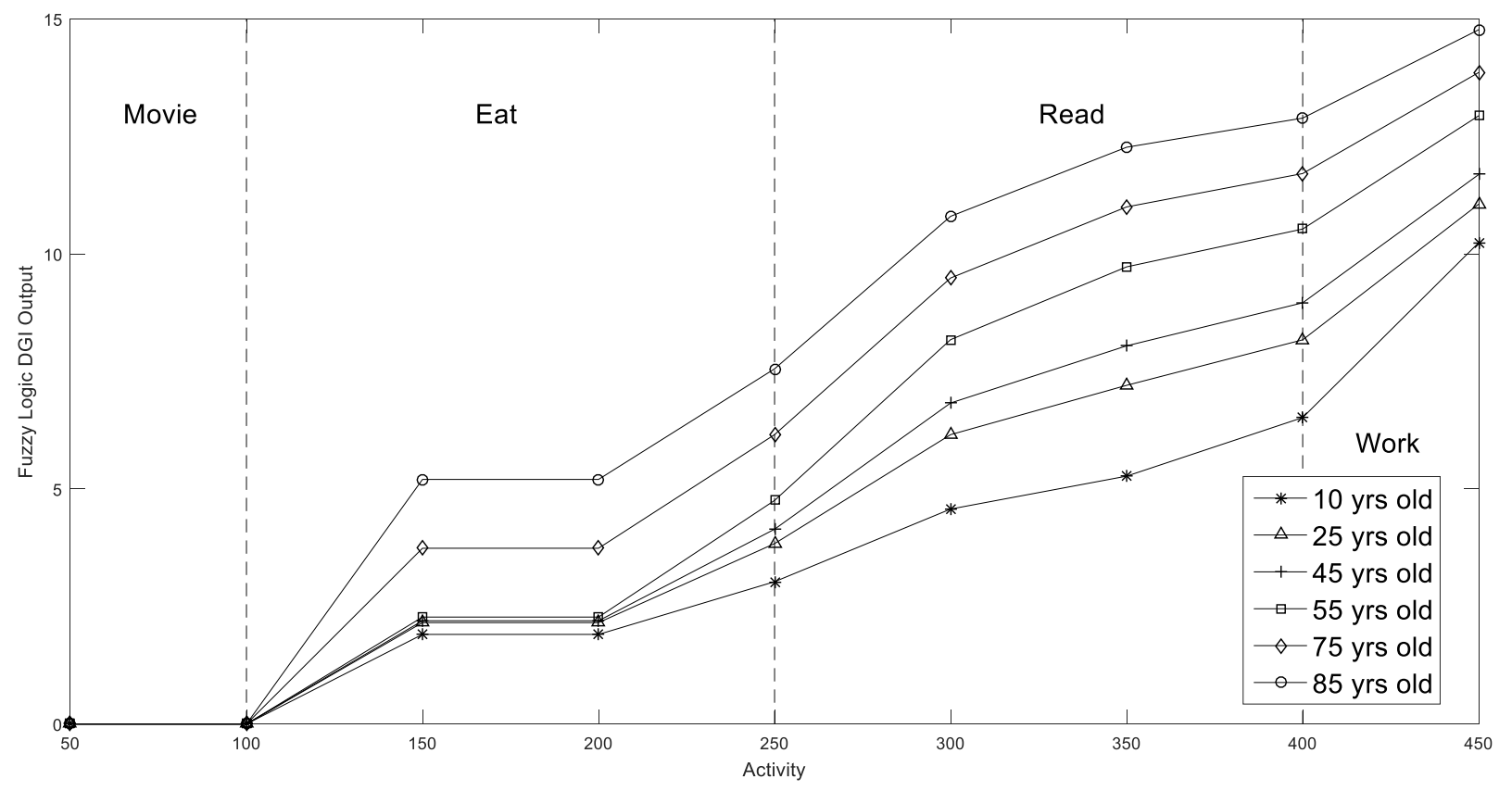

Figure 5.20 Result from fuzzy logic DGI output for various ages after adjustment

In addition to determining the DGISP based on age and activity, adjustments can be made by adding a suitable bias value towards the DGISP based on the user's eye condition as mentioned in section 5.2. The method of achieving this can be the same as that of determining the DGIsP by using ANFIS. However, since no actual data is available, it is not necessary to 
repeat the same discussion that would be more or less of similar procedure. The main point is that after having multiple eye condition inputs and corresponding membership functions, one single value should be determined, and this value, ideally a relatively small value, would be added to or subtracted from the DGIsp.

Lastly, Figure 5.21 shows the complete glare control system which consists of the personalization and user control elements, as well as the fuzzy PD controller described in chapter 4.

In addition, an artificial lighting control component, which outputs illuminance and color temperature values is added. The automated artificial control assumes different light settings for occupant's age and the activity the user is performing. This will be discussed in the next section. 


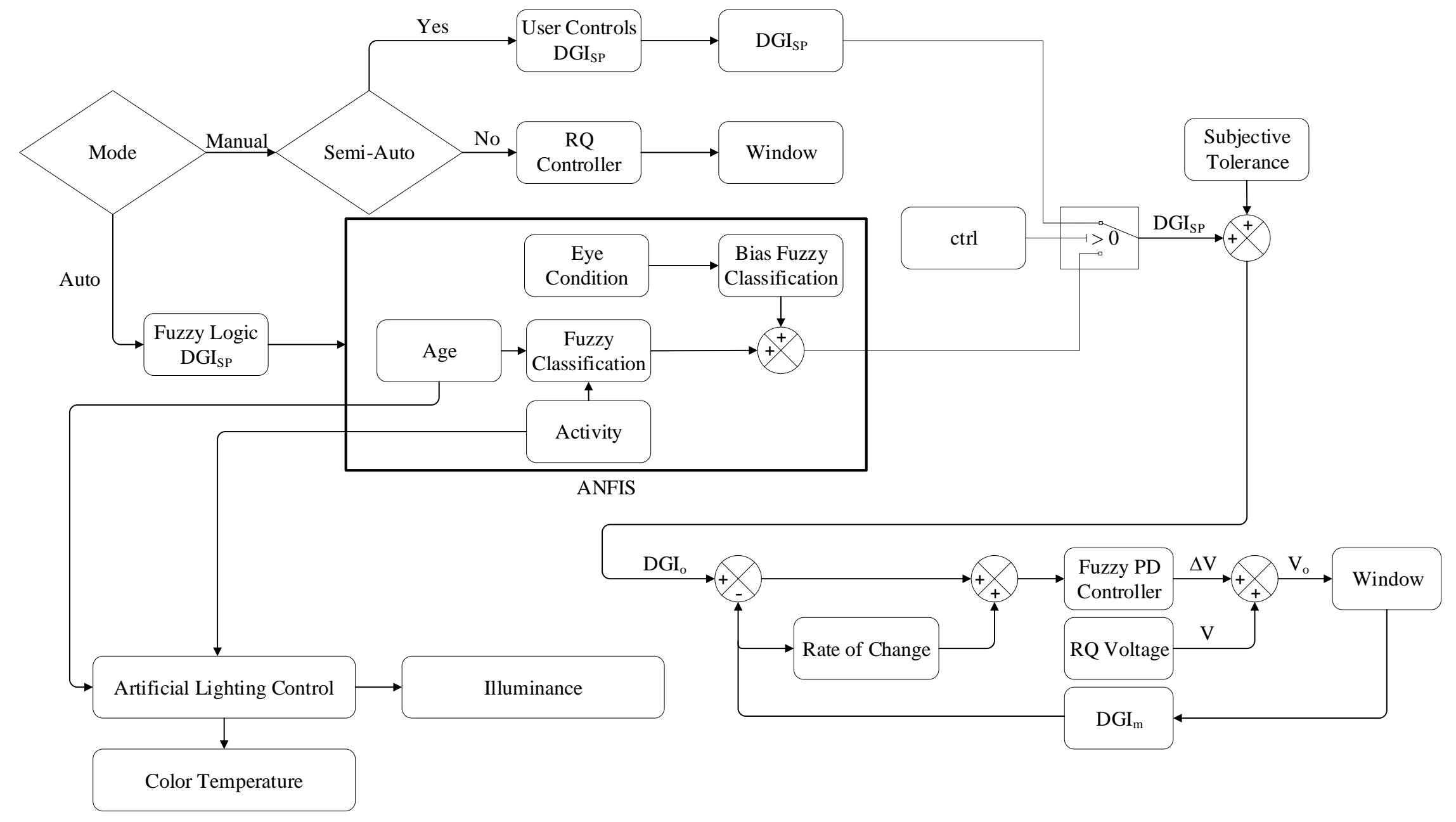

Figure 5.21 Complete glare control system 


\subsection{Investigation of Horizontal Illuminance and Color Temperature}

The control aspect of artificial illuminance has been discussed by plenty of scholars, some of which were mentioned in chapter 2. In this thesis, it is assumed that the automated artificial lighting control system which can output various illuminance levels is already developed. The control of horizontal illuminance is therefore not a main focus, rather the relationship of it with color temperature worth a dedicated discussion to maximize passenger visual comfort. During the investigation of the two factors by other scholars, they usually conducted the research in an area where the room is relatively spacious compared to aircraft cabin, and the type of activities that were performed were usually unique, such as that in office area, bedroom etc. What makes the scenario in a cabin unique is that it is generally a very confined space, and each personal area is relatively small, with limited daylight contribution. Either because of the window size or because of the prolonged darkness during flight, artificial lighting is usually heavily relied on. Therefore, the choice of illuminance and color temperature is going to be investigated using the mock up, and the method of doing so is to ask participants to adjust to their preference and notice if there is any pattern that can be seen.

\subsubsection{Horizontal Illuminance Versus Color Temperature Data Collection Method}

With the mock up environment, seven volunteers (four male and three female subjects age from 26 to 55) were asked to sit under the well-lit area with light sensor placed on the table $(66 \times 51 \mathrm{~cm})$ for data collection, it was confirmed that regardless of the location of the sensor being placed, the measured illuminance was almost the same, with the difference of only 10 lux. Therefore, the sensor was placed at the far end of the table that would not obstruct the participants actions during the experiment. 
During the start of the experiment, almost all external light sources were turned off, except for the emergency lighting that could not be turned off, leaving the mock up as dark as it could possibly be. With the cloth shielding from the external, it was measured at 9 lux without any contribution of mock up artificial lighting. The participants were then instructed to perform the tasks as mentioned in the previous section and in the following sequence.

1. Movie - Participants were asked to watch a short video for few minutes on their smart phones. This mimics the aircraft passengers watching movies on their own digital screens during flight. The idea was to let the passengers focus on their screens in a relaxed manner. Then they were asked to adjust the illuminance and the color temperature to a level that they think would the best for themselves through a smartphone application.

2. Read - Participants were given two articles, which were printed in black and white so that the contrast of the words and background would not become a factor in lighting adjustment. They were not required to finish reading the entire article, instead, they were asked to signal whenever they were satisfied with the lighting adjustments that they made by themselves.

3. Work - The difference between "work" and "read" is the different level of concentration required on performing the task. In order to characterize this, participants were asked to complete a sample IQ test with 25 questions and to be completed within 15 minutes. This length of time was chosen so that they would feel a little stressed to complete the test in short time, while not too long to cause annoyance for participating in this research since negative emotions might have an effect on the choice of lighting. Since this test was not meant for assessing their intelligence, the answers were not reviewed or graded and were shredded and disposed of immediately after the test. 
4. Eat - Participants were asked to bring their own favorite food for the final test. After the intense feeling of writing an IQ test, they were asked to relax and enjoy their food while sitting in the mock up cabin.

\subsubsection{Results of Participants' Selections and Their Verbal Responses}

The following figure shows the recorded data from the participants' selections. Due to the limitation of the light source, they were only allowed to adjust color temperature from $2000 \mathrm{~K}$ (usually the lowest color temperature that were being used for investigation) to $6535 \mathrm{~K}$ (with $6000 \mathrm{~K}$ being the color temperature of overcast sky [36]).

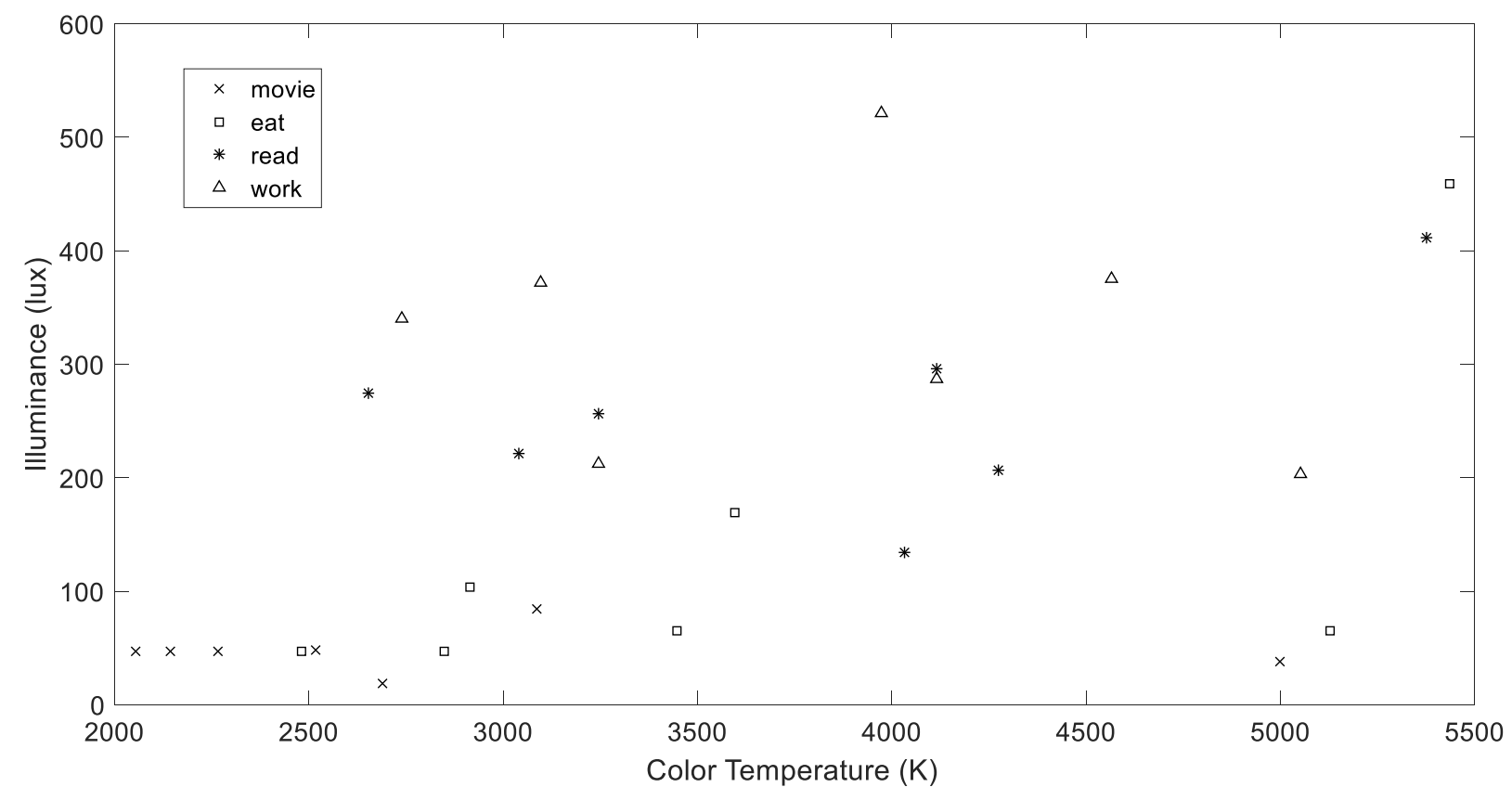

Figure 5.22 Participants' selection of illuminance and color temperature for various activities

In terms of illuminance, participants' responses showed very close resemblance of illumination selections with respective to the indoor lux value table listed in chapter 2 , and the same table is listed as follows as well for comparison. When watching movies, all participants chose illuminance level below 100 lux, and for eating, the trend of level was slightly higher, 
between 50 to 170 lux, except for one participant that desired 460 lux for eating in which the reason provided was the preference to look at the food clearly.

Table 5.7 Illuminance values (lux) at various indoor scenarios

\begin{tabular}{|c|c|c|c|c|c|}
\hline Scenario & Lux & Scenario & Lux & Scenario & Lux \\
\hline Parking lots & 50 & Corridor & $100-150$ & Dining rooms & 200 \\
\hline Library & 300 & Offices & $400-500$ & Art offices & 750 \\
\hline Electronics assembly & 1,000 & Hand tailoring room & 2,000 & & \\
\hline
\end{tabular}

In terms of desired illuminance level for working and reading, there seemed to be an overlapping area around $200-300$ lux. However, the range of difference was larger working compared to reading. This range also matched the trend in Table 5.7. However, it is shown that only one participant desired more than 500 lux for working while the others preferred less than 400 lux.

For color temperature, none of the participants chose color temperature higher than 5500 $\mathrm{K}$. When watching movie, generally participants selected very low color temperatures between $2000 \mathrm{~K}$ to $3100 \mathrm{~K}$. The participant that chose $5000 \mathrm{~K}$ for this activity was the same person that chose high illuminance level for eating. It may suggest dim environment might not be preferred for him at all and is treated as a subjective preference. For that of reading and working, the color temperature that most participants desired were generally slightly lower than that of while working, with the range of $2500 \mathrm{~K}$ to $4250 \mathrm{~K}$ compared to $2700 \mathrm{~K}$ to $5000 \mathrm{~K}$ while working.

\subsubsection{Discussion of Results of Participants' Responses}

From the results of illuminance levels, it can be seen that for low to medium concentration intensive tasks, the results were generally in agreement with the values suggested by literatures. However, for concentration intensive task, which is the "work" category, 
participants generally chose slightly lower level by 100 lux compared to literature. This can be due to the fact that the cabin area was small, and while they were working, the difference between peripheral luminance and central luminance was large, leading to a higher emphasis on central vision and therefore lower horizontal illuminance was required.

The reason that the participants gave towards the fact that none of them chose a color temperature higher than $5500 \mathrm{~K}$ was because they thought that the blue hue that appeared in high color temperature was irritating while they read or work. However, the light source that was used was not a certified photometrically calibrated device, blue hue that the participants experienced might be too strong compared to what it actually is. Nonetheless, it is interesting to notice the blue hue that scholars claim to increase alertness might actually be undesirable for cabin setting.

The other major difference between working and reading as seen from Figure 5.22 is the large range of preferred color temperatures chosen by the participants. This issue had been discussed by Priest [53] in 1933 who just suggested that human eyes do not respond well towards color temperature. Rather we are more responsive to the reciprocal of color temperature, which is called Mirek M. Therefore, a more useful way of noticing the pattern of participants' preference pattern is to plot illuminance versus Mirek as shown in Figure 5.23. In contrast with Figure 5.22, the color temperature was interpreted oppositely from the left to right, since the lower the Mirek value, the higher the color temperature is. The Mirek value is expressed as:

$$
M=\frac{1}{\text { color temperature }} \times 10^{6}
$$

By interpreting participant behaviors using Figure 5.23, more patterns can be noticed. The first thing to notice is that no participants, under any circumstances chose low color 
temperatures with illuminance level higher than 50 lux when Mirek value was higher than 400 $\mathrm{MK}^{-1}$. This matches with literatures suggestions that low color temperatures are not preferable at high illuminance level.

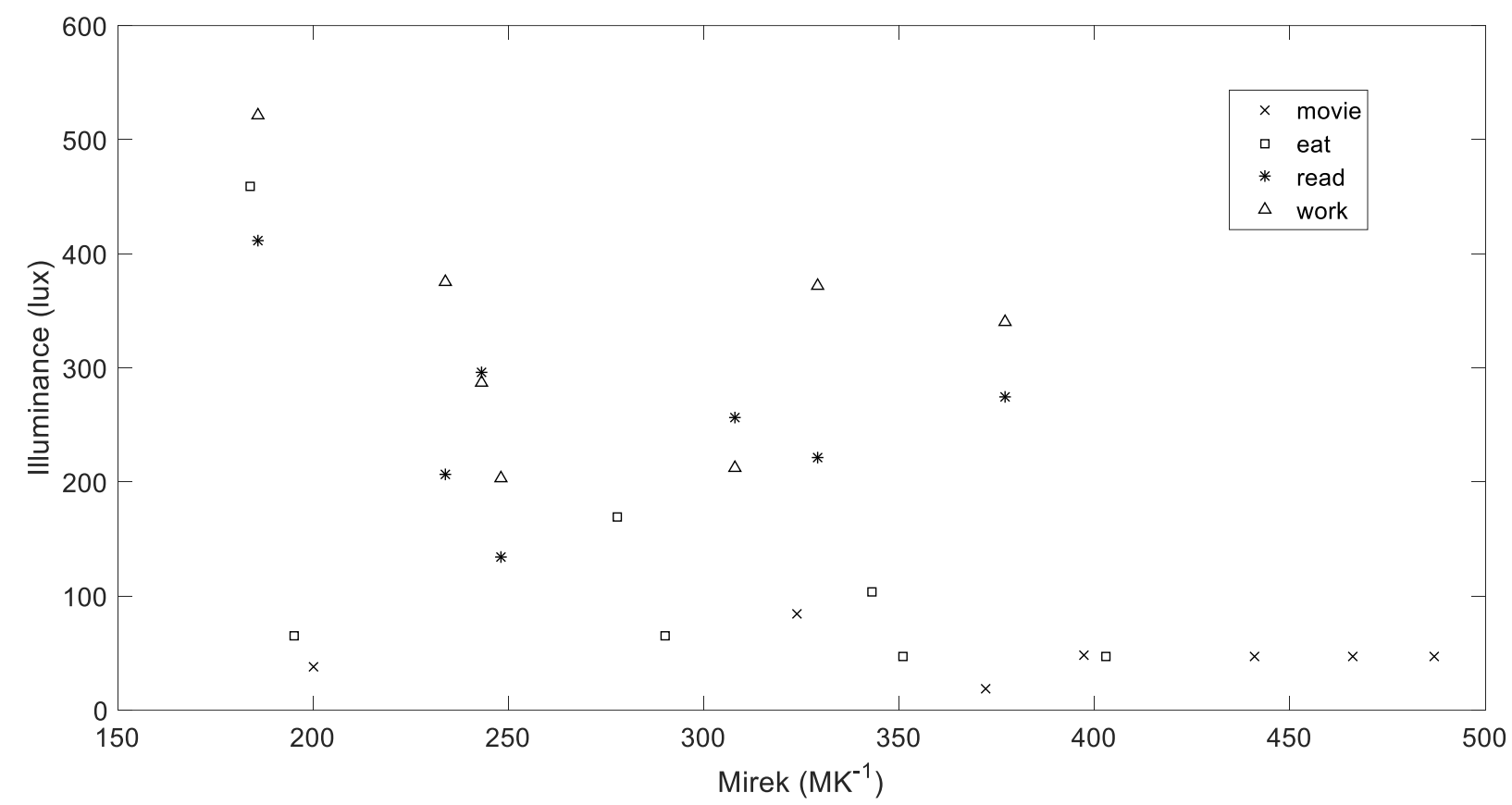

Figure 5.23 Participants' selection of illuminance and color temperature for various activities plotted with Mirek

The second area to notice is while the reading and working, the majority of responses were between 250 to $350 \mathrm{MK}^{-1}$. Within this range, there was no significant evidence showing any strong relationship between color temperature and illuminance. Participants did mention that increasing the color temperature made that environment looked brighter while they did not change the illuminance level. This gives the clue that within this area, participants might actually be finding the suitable illuminance level by adjusting color temperature and vice versa.

It is generally understandable that when eating, the required luminance is not that high. However, from the results, it was seen that some of the participants chose some very low values which were the same settings from some of the restaurants that put emphasis on customer's 
dining mood. The reason that participants chose to dim the light might be due to the fact that they were allowed to do anything that they would desire to do when they eat in flight. As all participants had never been on a business aircraft before, their experiences were based on general commercial flight, in which, it was noticed that all participants continued to watch videos. This can explain why the preferred illuminance values for eating were so close to that when they were watching movies.

In terms of the consideration of color temperature into lighting system into a cabin environment, in order to increase visual comfort from the artificial lighting aspect, it is of course best to offer a light source that is able to vary in brightness and color temperature, and at the same time, record the user's preference of color temperature, so the lighting control system can adjust the luminaire to change the color temperature according to the desired illuminance. If this not possible, based on Figure 5.23 and user responses, two separate color temperatures should be offered- very warm white light (below $2500 \mathrm{~K}$ ) that is adjustable to illuminate the target area up to 100 lux, and white light ( $2800 \mathrm{~K}$ to $4000 \mathrm{~K}$ ) that is adjustable to illuminate the target area up to 500 lux, to accommodate different activities.

In view of the results obtained from participants, the above described categorization can be represented graphically with an illuminance versus Mirek diagram (Figure 5.24). The top right corner represents the undesired region of low color temperature with high illuminance settings, which is the same as most literature works. Within the diagram, areas can be further divided into regions that require various illuminance and color temperature combinations, for example, movie, eating, read and work regions. Furthermore, two other regions can be categorized by the preference of daylight white and bright light, and also daylight white and dim light. Although with limited data, the categorization is rather of a rough work, it does resemble 
that major characteristics that the field generally agrees on. For example, color temperature at fine dining hall is approximately $2700 \mathrm{~K}$ to $3500 \mathrm{~K}\left(370 \mathrm{MK}^{-1}\right.$ to $\left.285 \mathrm{MK}^{-1}\right)$ according to various sources, and this is well represented by the curve within the "eat" category.

Compared to the Kruithof's curve (Figure 2.8) that was proposed for evaluating visual comfort, it is believed that this proposed categorization of visual comfort is more direct, extensive and easier to understand. A lot of scholars that used the Kruithof's curve knowing that it is not accurate and too brief for visual comfort analysis still used the graph because there is no better option. If this method is further being developed, the usability and the area of application can be enhanced.

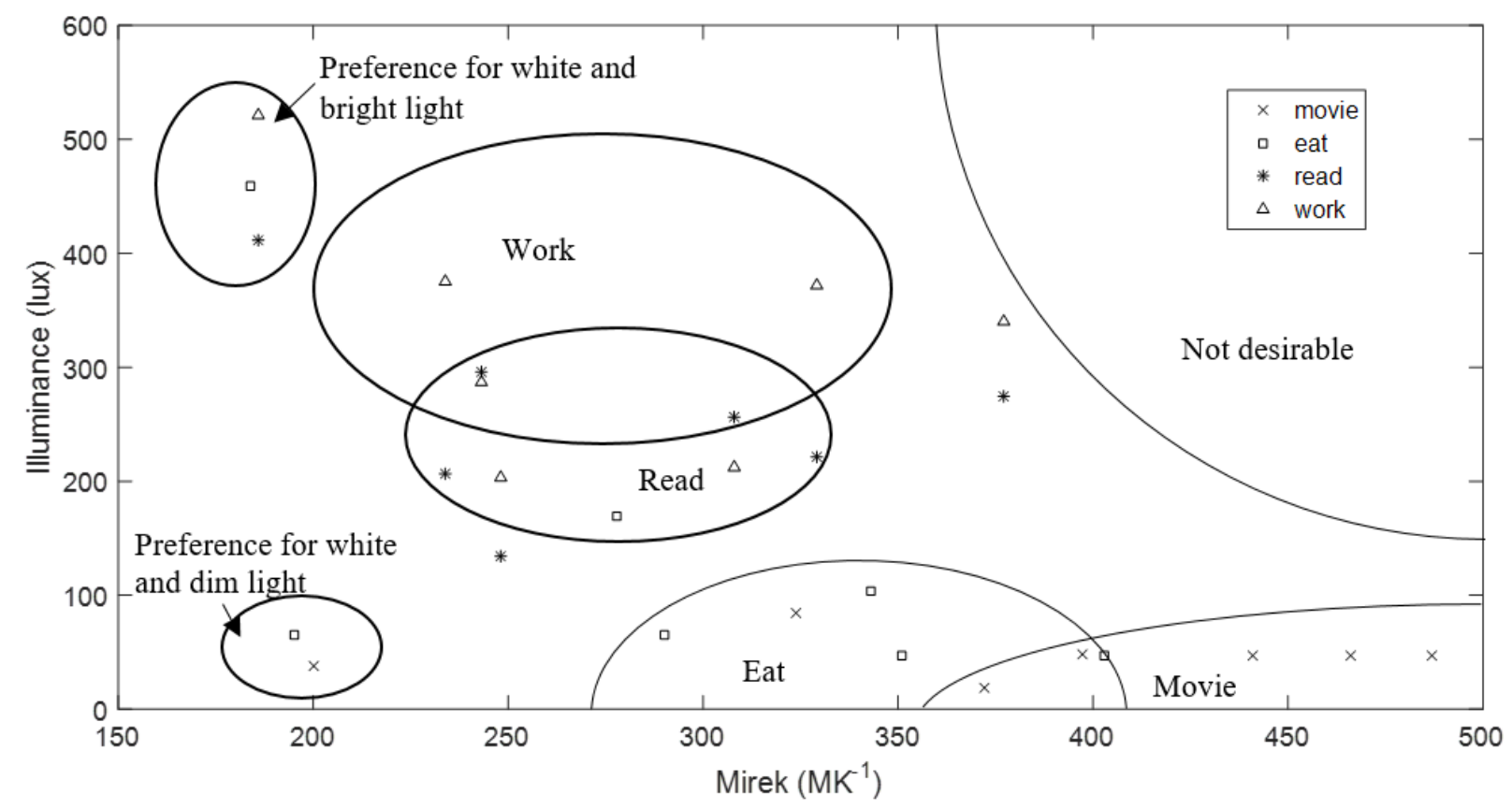

Figure 5.24 Proposed method of evaluating comfort using illuminance vs Mirek diagram

\subsubsection{Source of Errors}

The investigation of the preference of color temperature was only obtained from 7 participants from the age of 26 to 55 . The results showed some basic patterns, the more data 
there is for analysis, the clearer and convincing the conclusion can be. On the other hand, the light source that was used was not a photometrically calibrated device, in which the precision of color temperature might affect the participants' perceptions. However, it is believed that the overall difference would not be significant to overrule the entire results. With the chromaticity diagram that manufacturer distributed, the light source was well capable of representing color temperature from 2000 to $6535 \mathrm{~K}$. In Figure 5.25, the triangle that the arrow is pointing at is the range colors that the light bulb is able to produce.

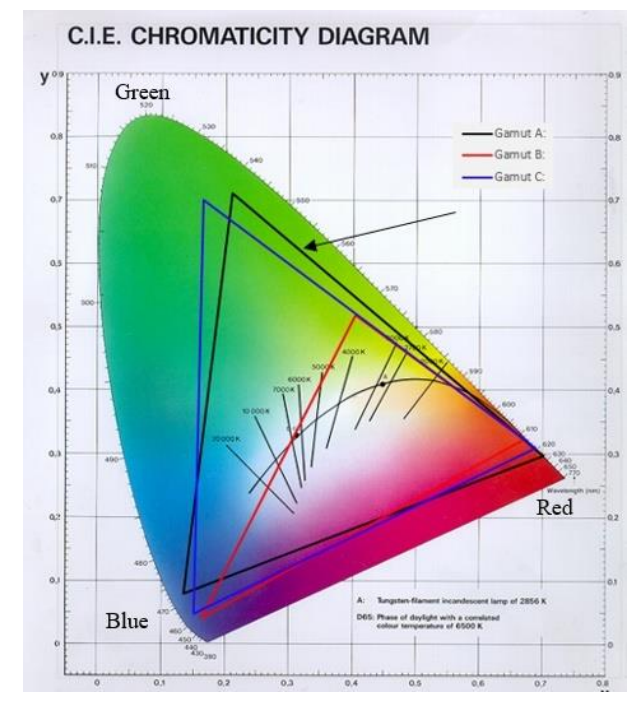

Figure 5.25 Chromaticity diagram of Philips hue A19 light bulb

On the other hand, the experiment was conducted through a mock up cabin, in which the effect of the surrounding had been neglected. A test in a real aircraft cabin should be conducted to see if interior reflection and overall color of the cabin may be elements to affect a person's choice of lighting. Lastly, during the reading test, the font size and line spacing of the articles that were given were different, this might cause confusion to older participants to make final decision when the value they chose were different for different articles. 


\subsection{Calculation of DGI}

Chapter 3 described the placement of the sensors, and this section presents the calculation of DGI $I_{m}$.

$$
D G I=8 \log _{10}\left\{0.25\left\{\frac{\sum\left(L_{\text {exterior }}^{2} \Omega_{p}\right)}{L_{\text {adaptation }}+0.07\left(\sum\left(L_{\text {window }}^{2} \omega\right)^{0.5}\right)}\right\}\right\}
$$

where $\omega$ is the solid angle subtended by the window to the point of observation (sr),

$$
\begin{gathered}
\omega=\frac{a b \cos \left(\tan ^{-1} X\right) \cos \left(\tan ^{-1} Y\right)}{d^{2}} \\
X=a / 2 d \quad Y=b / 2 d
\end{gathered}
$$

$a$ is the width of the window (m), $b$ is the height of the window (m) and $d$ is the perpendicular distance from the window to the sensor shielded by pyramid.

$\Omega_{p}$ is the solid angular subtence of the source modified for the effect of the position of its elements in different parts of the field of view (sr).

$$
\Omega_{p}=2 \pi \phi_{i}
$$

where $\phi_{i}$ is the configuration factor of the window from the observation place.

$$
\phi_{i}=\left(\operatorname{Atan}^{-1} B+\operatorname{Ctan}^{-1} D\right) / \pi
$$

where,

$$
\begin{array}{ll}
A=X /\left[\sqrt{1+X^{2}}\right] & B=Y /\left[\sqrt{1+X^{2}}\right] \\
C=Y /\left[\sqrt{1+Y^{2}}\right] & D=X /\left[\sqrt{1+Y^{2}}\right]
\end{array}
$$


The light sensor is capable of detecting vertical luminance $E_{v}$ from various sources with the unit of lux (lx). However, the unit that is required for calculation of DGI is $\mathrm{cd} / \mathrm{m}^{2}$. Therefore, the following conversion is required:

$$
\begin{gathered}
L_{\text {window }}=E_{v 3 \text { shielded }} /\left(2 \phi_{i} \pi\right) \\
L_{\text {adaptation }}=E_{v 2 \text { unshielded }} / \pi \\
L_{\text {exterior }}=E_{\text {vunshielded }} /[2(\pi-1)]
\end{gathered}
$$

Table 5.8 Values for DGI parameters

\begin{tabular}{|c|c|c|c|}
\hline Parameter & & Parameter & \\
\hline$a$ & $0.18 \mathrm{~m}$ & $X$ & 0.1343 \\
\hline$b$ & $0.36 \mathrm{~m}$ & $Y$ & 0.2687 \\
\hline$d$ & $0.67 \mathrm{~m}$ & $\omega$ & $0.1382 \mathrm{sr}$ \\
\hline$A$ & 0.1184 & $B$ & 0.2368 \\
\hline$C$ & 0.2118 & $D$ & 0.1059 \\
\hline$\phi_{i}$ & 0.0159 & $\Omega_{p}$ & $0.0998 \mathrm{sr}$ \\
\hline
\end{tabular}

\subsection{Re-location of Light Sensors}

Chapter 3 discussed about the placement of light sensors suggested by Nazzal [7], however, this thesis based the environment in an aircraft cabin, which means the current locations light sensors 2 and 3 were not practical in reality that they would be blocking the corridors and they were going to cause major inconvenience for anyone that would be using the area. Except for light sensor 1, which was placed right beside the window and should theoretically not going to be disruptive to passengers. 


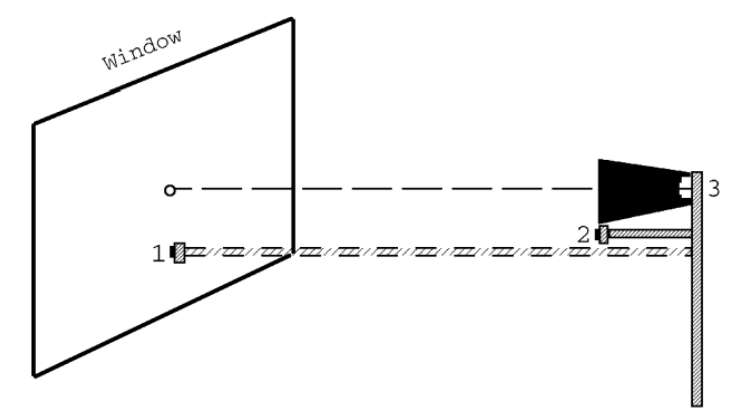

Figure 5.26 Placement of the three sensors and the pyramid suggested by Nazzal [7]

Light sensors 2 and 3 work in pair to calculate DGI, the relocation of light sensors would not change the fact that light sensor 3 had to be shielded and sensor 2 was still placed right under the opening of light sensor 3's shield opening.

There were two factors that were investigated - the distance $s$ of the pair of light sensors from the window, and the angle $\theta$ that the centerline of sensor 3, pointing towards the center of the window, makes with the $x$-axis. The maximum distance $s$ that was conducted was 70 inches from the window, and the maximum angle $\theta$ was $20^{\circ}$, where these were the physical limitations of the equipment and the cabin mockup environment. During the measurement process, instead of measuring angle $\theta$ directly, the distance $s$ ' and the height $p$ were adjusted accordingly.

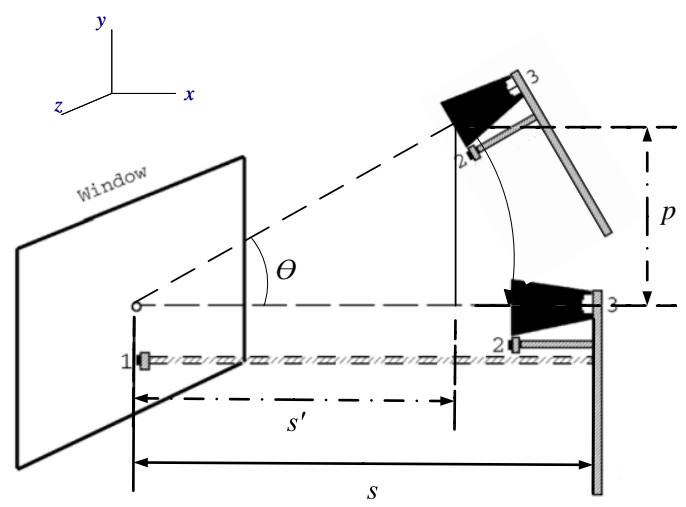

Figure 5.27 Re-location of sensors 2 and 3 
During the consideration of re-location, it was assumed that the most practical location to place the pair of sensors was on the ceiling of the cabin, opposite of the window that the sensors were detecting and before the lighting equipment of the opposite side of the cabin from the target window. If these three criteria were met, then firstly, the sensors would not be in the way of any passengers. Secondly, since the light sensors were spot sensors and the surrounding lighting system was only beside or behind them, the influence of irrelevant artificial lighting on detecting the DGI for the target passenger could be reduced. The trapezoid that is circled in Figure 5.28 shows one of the suggested placements of the sensors and it is directed towards the center of the target window.

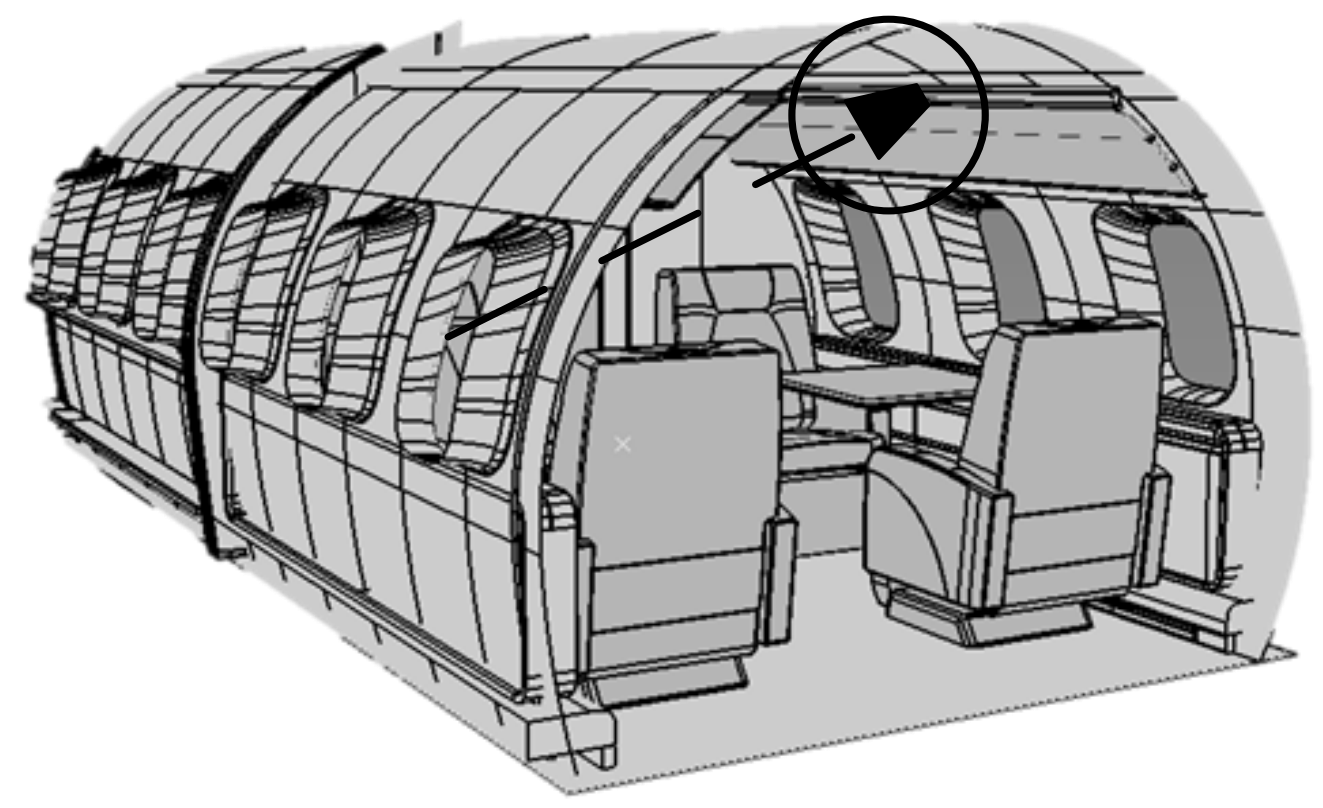

Figure 5.28 Suggested placement of light sensor pair in Bombardier Aerospace Global 7000 cabin environment

\subsubsection{Changing Angle of Light Sensor Pair}

Figure 5.29 shows the measurement results of moving the sensor pairs starting from 45 inches to 70 inches to the window. Finding the relationship of DGI vs angle $\theta$ closer than 45 inches is not necessary, since the adjustment of the height $\mathrm{p}$ corresponding to each angle was too 
small that it did not pose significant difference compared to placing the sensors right at the horizontal. However, on the other hand, regardless of the distance from the window, adjusting $\theta$ did not change the DGI values significantly. All are within 1 unit maximum. It was suggested it is not noticeable by users for difference within 1 DGI unit. If the sensors were replaced at say $20^{\circ}$ at the distance of 70 inches, the effect of it is that the window control system will dim the window darker by 0.33 unit. Therefore, it is not necessary to make changes towards the DGI calculation for placing the sensors at difference angles, at least if it was less than $20^{\circ}$.

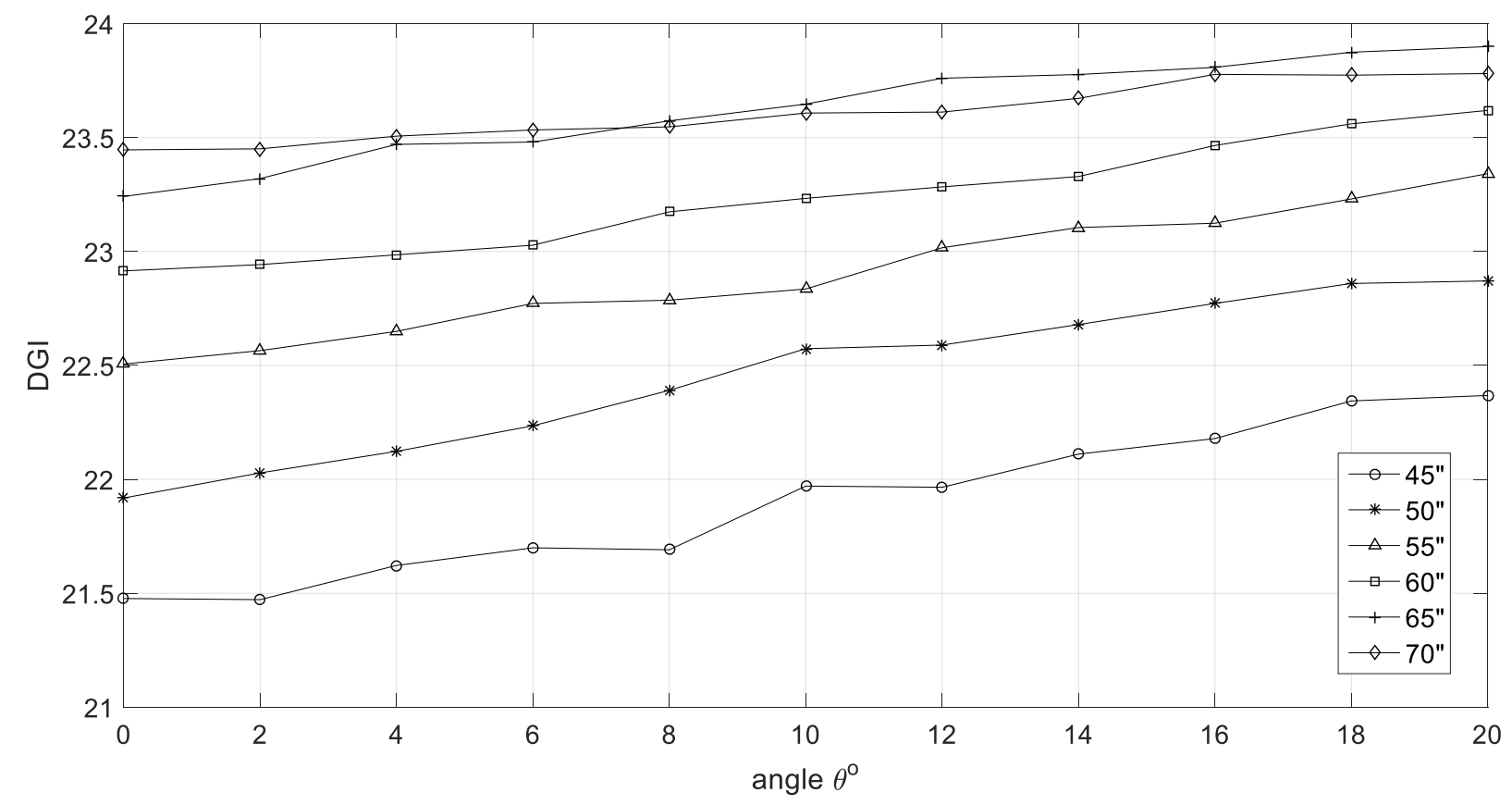

Figure 5.29 Effect of changing sensor angle from $0^{\circ}$ to $20^{\circ}$ and from distance of $45^{\prime \prime}$ to $70^{\prime \prime}$ under same lighting conditions

Table 5.9 Maximum DGI difference for angle adjustment at various distances

\begin{tabular}{|cc|cc|}
\hline distance $s$ & Maximum DGI difference & distance $s$ & Maximum DGI difference \\
\hline $45^{\prime}$ & 0.8912 & $60 "$ & 0.7046 \\
$50 "$ & 0.9527 & $65^{\prime}$ & 0.6575 \\
$55^{\prime}$ & 0.8356 & $70 ”$ & 0.3351 \\
\hline
\end{tabular}




\subsubsection{Changing Distance of Light Sensor Pair}

Changing distance of the pair of the sensors on the other hand, significantly changes the DGI values. For the following tests, the color temperature of the light sources was maintained the same while a few different brightness levels were evaluated. Also, angle $\theta$ was always kept at $0^{\circ}$. The discussion starts with Figure 5.30 having the external light source at maximum and setting the color temperature at maximum of $6535 \mathrm{~K}$, and the window always remained clear.
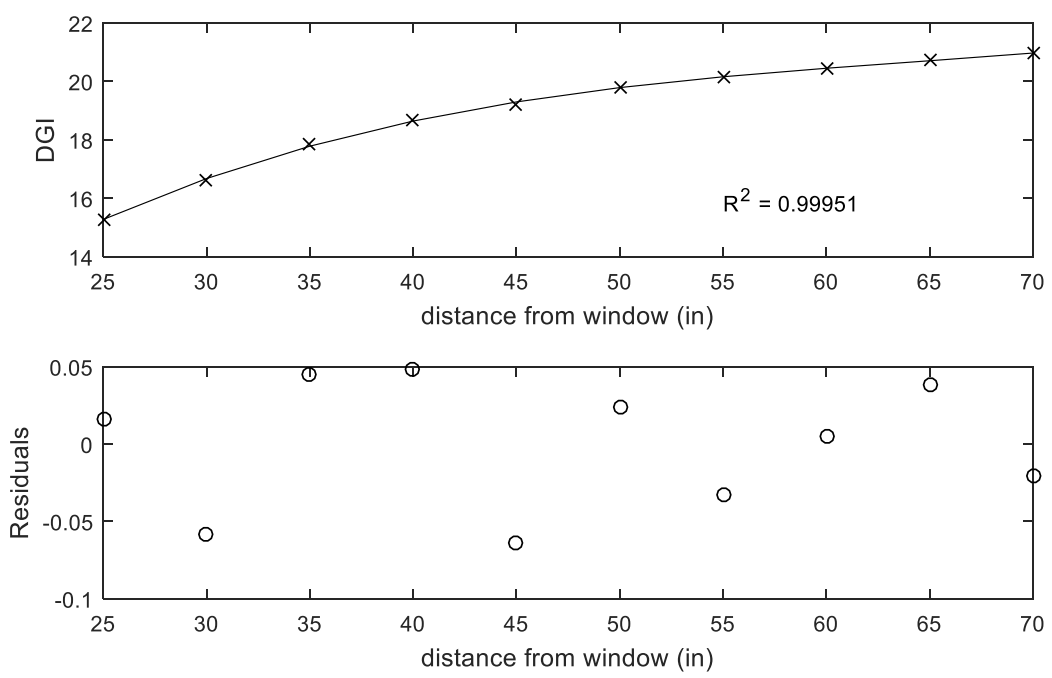

Figure 5.30 DGI vs distance at maximum brightness level and highest color temperature setting, $3^{\text {rd }}$ order relationship

The data collected was related with a $3^{\text {rd }}$ order relationship, the R-square value and residual plot confirmed that a $3^{\text {rd }}$ order polynomial is a suitable fitting compared to a $2^{\text {nd }}$ order relationship as shown in Figure 5.31 where the sine-wave pattern in the residual plot suggested that $2^{\text {nd }}$ order was not ideal regardless of the high $\mathrm{R}$-square value.

For several other brightness levels, from bright to dark, the same experiments were conducted, and the results, which were curve-fitted with respective $3^{\text {rd }}$ order equations are shown in Figure 5.32. 

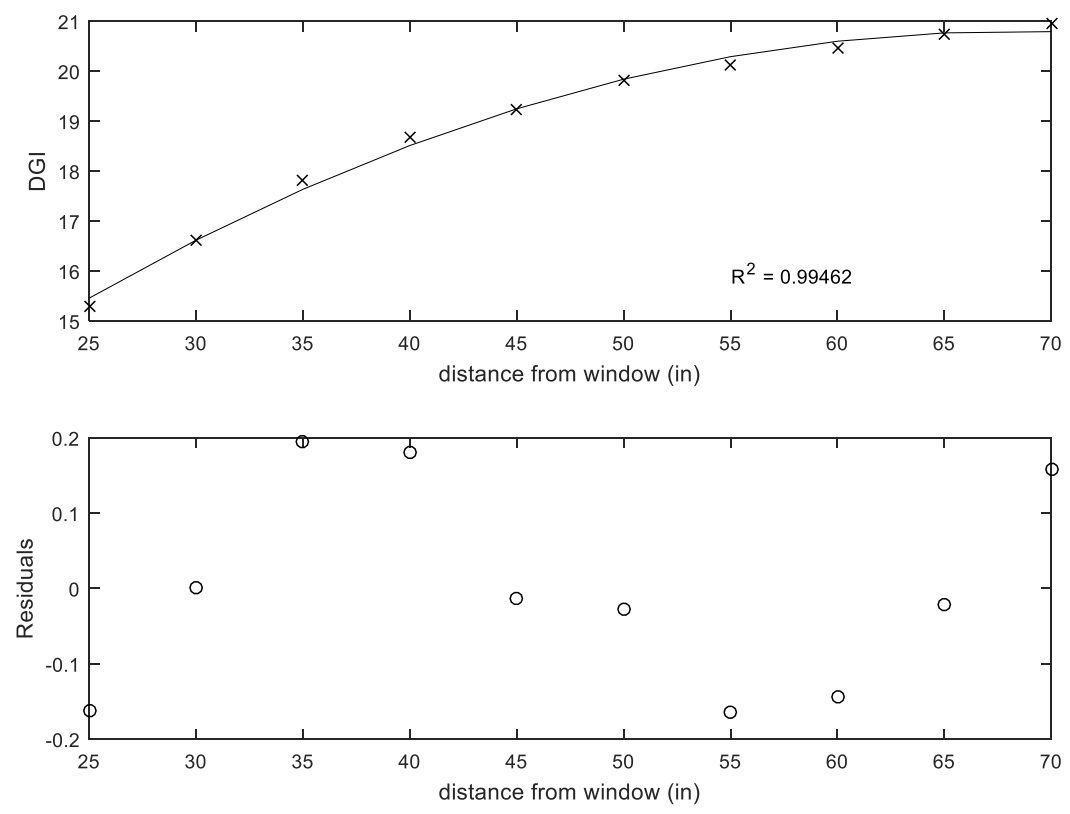

Figure 5.31 DGI vs distance at maximum brightness level and highest color temperature setting, $2^{\text {nd }}$ order relationship

The figures suggest that the sensor pair can be relocated at different distances that is perpendicular to the window, after the relocation, light sensor 1 now serves the purpose of choosing which correction curve is required to calculate the correct DGI. Suppose the sensor pair is relocated at a distance of 70 inches to 25 inches, and the detected DGI is 18 when the brightness level sensed by sensor 1 falls on to the curve DGI 254 which corresponds to 1352 lux. Then the respective equation is applied, substituting DGI as 18 and $\mathrm{x}$ is 70 , then $\mathrm{k}$ is calculated to be -0.5364 . Therefore, the actual DGI at 25 inches is 12.3 .

$$
D G I_{254}=5.6742 \times 10^{-5} x^{3}-0.010924 x^{2}+0.75145 x+k
$$

However, it is not possible or not practical to obtain all the curves for all brightness levels. Therefore, when the detected luminance level is between two curves, an interpolation is required to estimate a value, and linear interpolation is suggested to be sufficient to obtain a close value. 

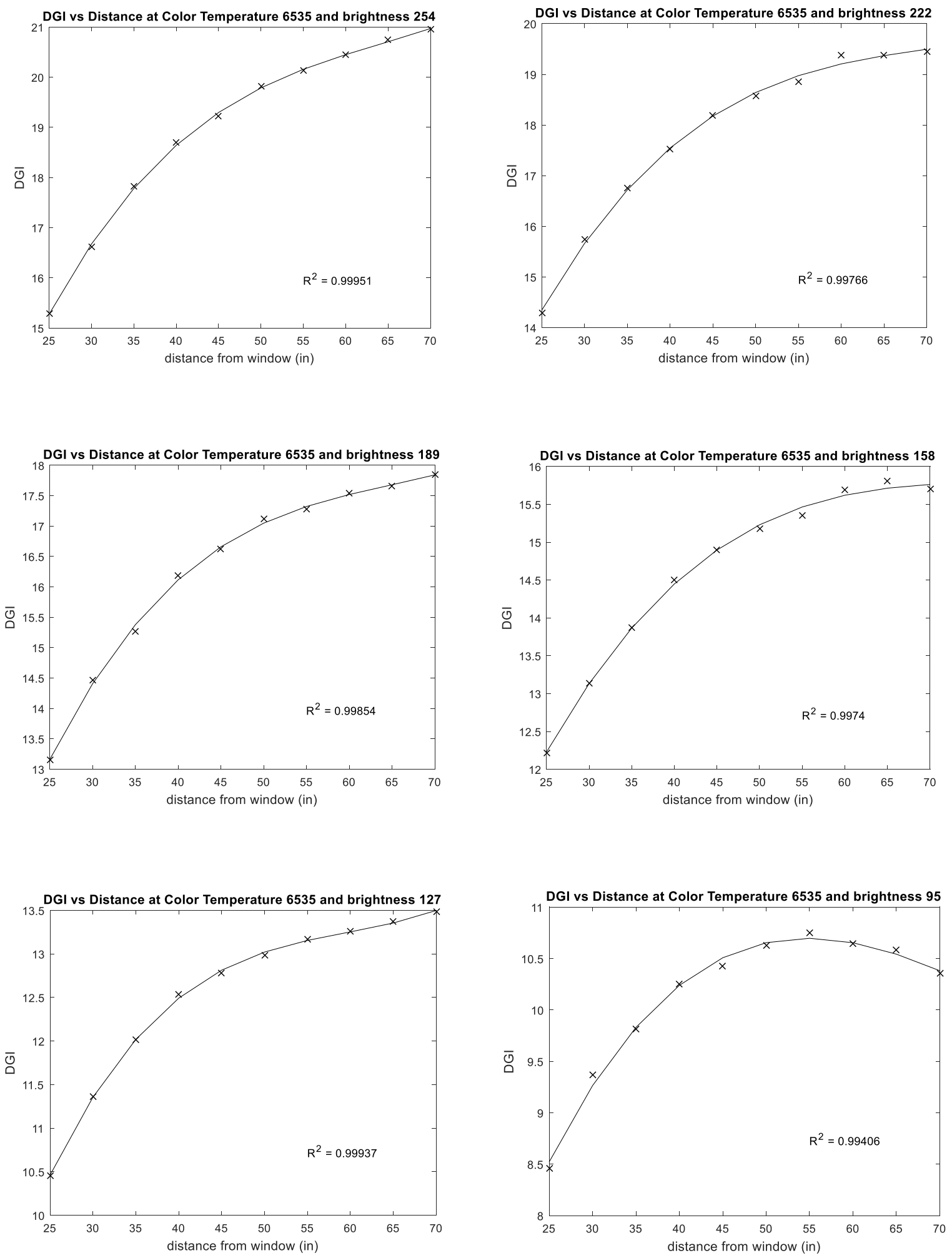

Figure 5.32 DGI vs distance at various brightness level and highest color temperature setting, $3^{\text {rd }}$ order relationship 
As a demonstration, suppose the DGI values at brightness level 222 is not known, while that of 254 and 189 are known, and now light sensor 1 detects a luminance value that falls on to the 222 curve, a linear interpolation is then required to calculate the proper DGI.

$$
D G I_{189}=5.2358 \times 10^{-5} x^{3}-0.010166 x^{2}+0.68884 x+1.4752
$$

The luminance of $\mathrm{DGI}_{222}$ is $1167 \mathrm{lux}$ and $\mathrm{DGI}_{189}$ is $956 \mathrm{lux}, \mathrm{DGI}_{(254 \mid x=25)}$ is 15.278 and $\operatorname{DGI}_{(189 \mid x=25)}$ is 13.161 .

$$
\begin{gathered}
D G I_{222_{e s t}}=\left[(1167-956) / \frac{1352-956}{15.278-13.161}\right]+13.161=14.289 \\
D G I_{222}(25)=4.2811 \times 10^{-5} x^{3}-0.0090783 x^{2}+0.66544 x+2.7142=14.345
\end{gathered}
$$

From the calculations, it can be seen that the linearly interpolated DGI value is very close to the DGI value obtained through the re-location curves shown in Figure 5.32. In addition, the entire linearly interpolated DGI curve is plotted as shown in Figure 5.33.
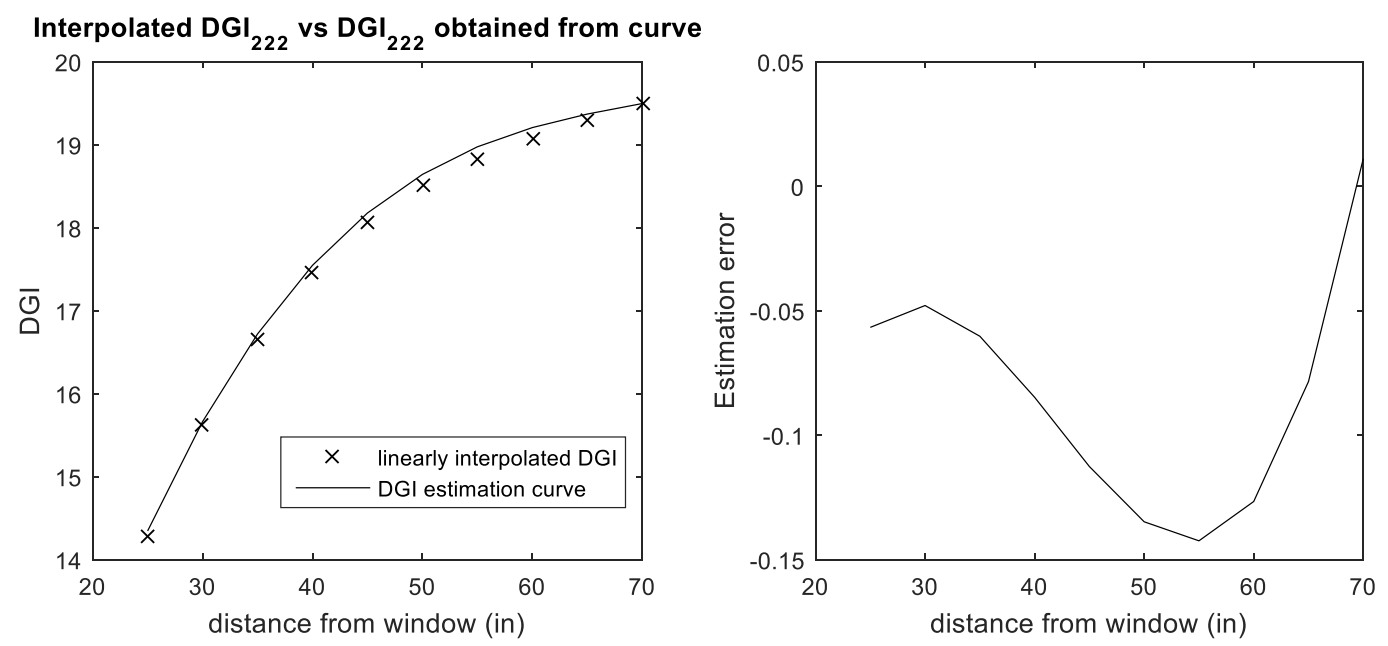

Figure 5.33 Linearly interpolated DGI vs DGI values obtained from estimation curves (left), and the interpolation error (right) 
The maximum magnitude of error for such interpolation method is 0.15 , which is largely within the always suggested boundary of negligible range of 1 unit. Therefore, the DGI estimation for various brightness using linear interpolations can yield the values deemed practically.

Knowing that fact that it is possible to relocate sensors to different positions, glare evaluation is made possible on the aircraft cabin using the DGI $_{\mathrm{N}}$ detection method. Combined with the fuzzy PD controller as discussed in chapter 4, this concludes the entire glare elimination method for aircraft cabin setting.

\section{Chapter 6 Conclusion}

This work started with the literature review of various work that were done by scholars that were related to visual comfort. Including illuminance, effect and people's preference of artificial light color and color temperature, and glare evaluation methods. Furthermore, the use of fuzzy logic on lighting system were studied. Through the review, it was found that glare evaluation methods, though widely accepted to be a standard way of demonstrating visual comfort, never had scholars agreed on systematic and standardized procedures. By considering the major factors that the glare indices were developed upon, $\mathrm{DGI}_{\mathrm{N}}$ was chosen to be parameter to evaluation visual comfort. With reference to the widely accepted Hopkinson's scale, the glare level as seen from the mockup of aircraft cabin was restricted to be below 22 .

Knowing the fact that glare indices were simply approximations, they were not guaranteed to suit every person, every environment and activity that the occupants performed. Therefore, the method of personalizing glare level was proposed, and it was aimed to provide 
certain amount of external light into the cabin, while the glare level, which was tailored towards each specific passenger, was maintained at the acceptable level.

On the other hand, the preference of color temperature of artificial lighting was conducted by having 7 participants to provide input, although the sample data is small, it provided the insight that the evaluation of the preference of color temperature might be different from what was usually preferred in more spacious area compared to an aircraft cabin, and it was suggested that warm white light that could illuminate the target area up to 100 lux and white light of up to 500 lux shall be taken into account of during the lighting design. Furthermore, it was proposed that instead of using Kruithof's curve for evaluating visual comfort in terms of color temperature and illuminance, the use of illuminance versus Mirek was a more direct and extensive graphical representation of visual comfort.

In order to allow external light into the cabin, traditional window shades are certainly not possible except when they are fully opened. Through the comparison of various smart window technologies, electrochromic window was chosen to be the suitable device to achieve the task. However, there has not been any control system developed that were dedicated towards electrochromic window. Therefore, a fuzzy PD controller was designed to change the tinting level of the window to ensure that the desired glare level was always maintained within error margin of 1 unit, which was believed that be unnoticeable by human eye.

Lastly, it was noticed that the placement of the equipment that were used to detect glare levels was not practical in an aircraft cabin setting as two of the three light sensors required were interfering with the limited space that could cause major disruption to all person on board. In order to solve this problem, the sensors were re-located to a better position on the ceiling of the aircraft, and the method to correct the deviated $\mathrm{DGI}_{\mathrm{N}}$ value was proposed. It was proven that the 
angle of placement the sensors was not major issue towards the DGI calculation, but the effect of change of distance needed to be corrected by using respective $3^{\text {rd }}$ order polynomials. Results showed that the difference between the original detected glare value and the corrected glare value was very small that they could be assumed to be negligible and therefore allowing the relocated DGI glare system to be used practically.

\subsection{Contributions}

The contribution of work falls within the four areas discussed in this thesis - glare detection and evaluation, fuzzy logic controller for electrochromic window, personalization of glare level and color temperature for various activities.

In terms of glare detection, $\mathrm{DGI}_{\mathrm{N}}$ was determined to be the most suitable method for an aircraft cabin setting to evaluate passenger visual discomfort. In order to allow the equipment to be placed conveniently in the cabin, a method of relocation and interpolation was discussed to maximize the practicality of the implementation of the system.

Electrochromic window was found to be the best solution for using on the aircraft given its high opacity. Automated control for the window using fuzzy logic PD controller was developed to largely reduce the need for manual input. Also, a method of personalizing the transparency of window to suit different passengers' needs based on the activity they were performing was proposed.

Lastly, a new plot for illuminance versus color temperature (in fact Mirek) was proposed in order to provide a more direct and extensive graphical representation of the two parameters as supposed to Kruithof's curve. 


\subsection{Future Work}

Throughout all the elements of lighting, in this thesis, only illuminance, glare and color temperature were investigated, with glare and color temperature being discussed in a brief manner. The inclusion of other factors such as color, effect of daylight being filtered into blue light after passing through the electrochromic window, effect of other glare sources far from the passenger's seat can all be investigated in the future.

The other major part of this research is the development of controller for electrochromic window, the major reason that fuzzy logic was chosen came from the classification concept, however, eventually it was realized that a fuzzy PD controller was the required controller in terms of practicality. This controller method is beneficial to control the window as the dynamics of the plant is not fully understood. While work has been done on modelling electrochromic devices, but from the understanding of scholars' work, there were still major discrepancies between their models and actual behaviors due to non-linearity. Therefore, modelling the dynamics of electrochromic window can lead to a more sophisticated controller developed for it.

The investigation of illuminance and color temperature was conducted in a very brief manner, the test could be conducted in a way that participants actually sit inside a real aircraft cabin, and instead of using the color temperature values from the light source, certified calibrated color temperature meter can be used to ensure more accurate results, and therefore further enhancing the idea of using illuminance versus Mirek for visual comfort evaluation.

Lastly, there are problems related to using electrochromic window on airplane use, such as very long transition time that cause annoyance and brown residues appearing on windows due to hoop stress. Regarding the former problem, there are newer generations of electrochromic windows that have much faster response time and could eventually solve the problem as even 
newer generations become available. Regarding the latter problem, it requires dedicated investigation in order to solve it. 


\section{Bibliography}

[1]IATA. "IATA Forecasts Passenger Demand to Double Over 20 Years." IATA. N.p., 18 Oct, 2016. Web. [Accessed: 20-Octorber-2017]

[2]Vink P. and Brauder K., Aircraft Interior Comfort and Design. Boca Raton: CRC Press, 2011.

[3]Ochoa, C., Aries, M., Loenen, E. and Hensen, J. "Considerations on design optimization criteria for windows providing low energy consumption and high visual comfort." Applied Energy, vol. 95, June 2012, pp. 238-245., doi:10.1016/j.apenergy.2012.02.042.

[4]Mechanical window shade. Digital image. Upgrade: Travel Better. N.p., 12 May 2007. Web. 20 Feb. 2017.

[5]Electrochromic windows. Digital image. 360doc. N.p., 14 Aug. 2012. Web. 20 Feb. 2017.

[6]The Boeing Company, "Statistical Summary of Commercial Jet Airplane Accidents", Aviation Safety Boeing Commercial Airplanes, Seattle, Washington, 2015.

[7]Nazzal, A. “A new evaluation method for daylight discomfort glare.” International Journal of Industrial Ergonomics, vol. 35, no. 4, 20 Apr. 2004, pp. 295-306., doi:10.1016/j.ergon.2004.08.010.

[8]DiLaura, D., Houser, K., Mistrick, R. and Steffy, G. The Lighting Handbook. 10th ed., Illuminating Engineering Society, 2011.

[9]Shuster, J. "Addressing Glare in Solid-State Lighting." Ephesus, January 2014. Web. [Accessed: 8-Aug-2017]

[10]Cédric, A., Leclercq, M., Bideaux, E. and Flambard, L. "A Smart Sensor to Evaluate Visual Comfort of Daylight into Buildings." International Journal of Optomechatronics, vol. 2, no. 4, 11 Nov 2008, pp. 413-34, doi:10.1080/15599610802438672.

[11]Piccolo, A. and Simone, F., "Effect of switchable glazing on discomfort glare from windows", Building and Environment, vol. 44, no. 6, pp. 1171-1180, 2009., doi: 10.1016/j.buildenv.2008.08.013.

[12]Osterhaus, W. "Discomfort glare assessment and prevention for daylight applications in office environments." Solar Energy, vol. 79, no. 2, Aug. 2005, pp. 140-158., doi:10.1016/j.solener.2004.11.011.

[13]Huang, H., Li, C. and Yuan, Y. "Effects of Age and Ambient Illuminance on Visual Comfort for Reading on a Mobile Device." Color Research and Application, vol. 42, no. 3, June 2017, pp. 352-361., doi:10.1002/col.22089. 
[14]Tokura, M., Iwata, T. and Shukuya, M. "Experimental study on discomfort glare caused by windows - subjective response to glare from a simulated window." Journal of Architecture, Planning and Environmental Engineepring, no. 489, Nov. 1996, pp. 17-25., doi:10.3130/aija.61.17_8.

[15] Vainio, A., Valtonen, M. and Vanhala, J. "Proactive Fuzzy Control and Adaptation Methods for Smart Homes", IEEE Intelligent Systems, vol. 23, no. 2, pp. 42-49, 2008., doi: 10.1109/MIS.2008.33.

[16]Boyce, P.Human factors in lighting, 1st ed. London: Taylor \& Francis, 2003.

[17]Palmer, J. and Grant, B., The art of radiometry, 1st ed. Bellingham, Wash.: SPIE Press, 2010, pp. 1-9.

[18] McCluney, R., Introduction to radiometry and photometry, 1st ed. Boston: Artech House, 2014.

[19]Fernandes, L., Lee, E. and Ward, G. "Lighting Energy Savings Potential of Split-Pane Electrochromic Windows Controlled for Daylighting with Visual Comfort." Energy and Buildings, vol. 61, 13 October, 2012, pp. 8-20, doi:10.1016/j.enbuild.2012.10.057.

[20]Leclercq, M., Anthierens, C., Bideaux, E. and Flambard, L. "Design of a Visual Comfort Sensor for Daylighting Devices." Proc of 17th IFAC world congress, Seoul, Korea, 6 July 2008, pp. 6873-6878.

[21]Wienold, J. and Christoffersen, J. "Evaluation Methods and Development of a New Glare Prediction Model for Daylight Environments with the Use of CCD Cameras."Energy and Buildings 38.7 (2006): 743-57. Web., doi: 10.1016/j.enbuild.2006.03.017.

[22]Suk, J. and Schiler, M. "Investigation of Evalglare software, daylight glare probability and high dynamic range imaging for daylight glare analysis." Investigation of Evalglare software, daylight glare probability and high dynamic range imaging for daylight glare analysis, vol. 45, no. 4, 11 Sept. 2012, pp. 450-463., doi: 10.1177/1477153512458671.

[23]Lighting handbook: reference and application. Illuminating engineering society of North America IESNA. $9^{\text {th }}$ ed. New York: IESNA, 2000.

[24]L'eclairage, P. notions de base, projets d'installations. Londres: Tec \& Doc Lavoisier; 1993.

[25]"How Smart Windows Work?" [Online]. Available: https://home.howstuffworks.com/home-improvement/construction/green/smart-window2.htm. [Accessed: 25-Oct-2017].

[26]Takatoh, K., Sakamoto, M. Hasegawa, R., Koden, M., Itoh, N. and Hasegawa, M. Alignment Technology and Applications of Liquid Crystal Devices. Vol. 5, CRC Press, 2005. 
[27]Lee, E. and Tavil, A. "Energy and visual comfort performance of electrochromic windows with overhangs." Building and Environment, vol. 42, no. 6, June 2007, pp. 2439-2449., doi:10.1016/j.buildenv.2006.04.016.

[28]Gugliermetti, F. and Bisegna, F. "Visual and energy management of electrochromic windows in Mediterranean climate." Building and Environment, vol. 38, no. 3, Mar. 2003, pp. 479-492., doi:10.1016/S0360-1323(02)00124-5.

[29]Schubert, E.Light-emitting diodes, 1st ed. Cambridge, Uk: Cambridge University Press, 2003, pp. 275-288.

[30]"Spectral sensitivity of the human eye" [Online]. Available: https://lightmeasurement.com/spectral-sensitivity-of-eye/ [Accessed: 24-Sept-2017]

[31]Itten, J. The Art of Color: The subjective Experience and Objective Rationale of Color. New York: John Wiley, 2002.

[32]Winzen, J., Albers, F. and Marggraf-Micheel, C. "The influence of coloured light in the aircraft cabin on passenger thermal comfort." Lighting Research \& Technology, vol. 4, no. 46, 13 June 2014, pp. 465-75.

[33]Thapan, K., Arendt, J., and Skene, DJ. "An action spectrum for melatonin suppression: evidence for a novel non-Rod, non-Cone photoreceptor system in humans." The Jounral of Physiology, no. 535, 15 Aug. 2001, pp. 261-267.

[34]Valdez, P., and Mehrabian, A. "Effects of color on emotions." Journal of Experimental Psychology. General., vol. 4, no. 123, Dec. 1994, pp. 394-409.

[35]Smith, J. "Calculating color temperature and illuminance using the TAOS TCS3414CS digital color sensor." Intelligent OPTO Sensor Designer's Notebook, 27 Feb. 2009, www.taosinc.com.

[36]Chain, C., Dumortier, D., and Fontoynont, M. "A comprehensive model of luminance, correlated color temperature and spectral distribution of skylight: Comparison with Experimental Data." Solar Energy, vol. 65, no. 5, 1 Apr. 1999, pp. 285-295., doi:10.1016/S0038092X(98)00145-5.

[37]Sinoo, M., Hoof, J. and Kort, H. "Light conditions for older adults in the nursing home: Assessment of environmental illuminances and colour temperature." Building and Environment, vol. 46, no. 10, Oct. 2011, pp. 1917-1927., doi:10.1016/j.buildenv.2011.03.013.

[38]Ian, A. "The Kruithof Curve: A Pleasing Solution. Wordpress blog. 19 Mar, 2015.

[39]Kakitsuba, N. "Preferred Color Temperatures at 200 lx during Exposure to Cool or Warm Environments for Middle-Aged Female Subjects." Journal of the Human-Environment System, vol. 6, Jan. 2003, pp. 93-100., doi:10.1618/jhes.6.93. 
[40]Chain, C., Dumortier, D. and Fontoynont, M. "Consideration of daylight's colour." Energy and Buildings, vol. 33, no. 3, July 2007, pp. 193-198., doi:10.1016/S0378-7788(00)00081-5.

[41] Boyce, P. and Cuttle, C. "Effect of correlated colour temperature on the perception of interiors and colour discrimination performance." Lighting Research \& Technology, vol. 22, no. 1, 1 Mar. 1990, pp. 19-36., doi:10.1177/096032719002200102.

[42]Bodmann, H. "Quality of Interior Lighting Based on Luminance.” Lighting Research \& Technology, vol. 32, no. 1, 1 Mar. 1967, pp. 22-40., doi:10.1177/147715356703200104.

[43]Oi, N. and Takahashi, H. "Preferred combinations between illuminance and color temperature in several settings for daily living activities." Proc of 26th Session of the CIE, vol. 2, 6 July 2008.

[44]Saravanan, R. "Fuzzy Controller Design of Lighting Control System By Using VI Package." International Journal of Artificial Intelligence, vol. 1, no. 2, June 2012, pp. 73-78., doi:10.11591/ij-ai.v1i2.441.

[45]Javidbakht, S."Design of a Controller to Control Light Level in a Commercial Office." M.S.Egr. Thesis, Department of Electrical Engineering, Wright State University, pp. 1-106, 2007.

[46]Kolokotsa, D., Tsiavos, D., Stavrakakis, G., Kalaitzakis, K. and Antonidakis, E. “Advanced fuzzy logic controllers design and evaluation for buildings' occupants thermal-visual comfort and indoor air quality satisfaction." Energy and Buildings, vol. 33, no. 6, July 2001, pp. 531543., doi:10.1016/S0378-7788(00)00098-0.

[47]Singhvi, V., Krause, A., Guestrin, C. Garrett, J. and Matthews, H. "Intelligent light control using sensor networks." Proc of the $3^{\text {rd }}$ International Conference on Embedded Networked Sensor Systems, ACM, 2005.

[48]Yeh, L., Lu, C., Kou, C., Tseng, Y. and Yi, C. "Autonomous light control by wireless sensor and actuator networks." IEEE Sensors Journal, vol. 10, no. 6, June 2010, pp. 1029-1041., doi:10.1109/JSEN.2010.2042442.

[49]Cha, U. and Koller, D. "Utilities as random variables: Density estimation and structure discovery." Uncertainty in Artificial Intelligence Proceedings, 2000.

[50]Lah, M., Zupancic, B., Peternelj, J. and Krainer, A. "Daylight illuminance control with fuzzy logic.” Solar Energy, vol. 80, no. 3, Mar. 2006, pp. 307-321., doi:10.1016/j.solener.2005.02.002.

[51]Mukherjee, S. “An Approach to Predict Daylight Glare Using Nazzal's Daylight Glare Index Formula." International Journal of Engineering Research and Development, vol. 12, no. 10, Oct. 2016, pp. 50-58. 
[52]"Venetian Blinds" [Online]. Available: https://www.luxaflex.co.uk/products/venetian-blinds/ [Accessed: 1-Nov-2017]

[53]Priest, I. "A Proposed Scale for Use in Specifying the Chromaticity of Incandescent Illuminants and Various Phases of Daylight." American Journal of Ophthalmology, no. 23, Feb. 1933, pp. 41-45., doi:10.1364/JOSA.23.000041. 